DESY 12-096, DO-TH-12/19, SFB/CPP-12-38, LPN 12-056, arXiv:1208.6087[hep-ph]

\title{
The Theory of Deeply Inelastic Scattering
}

\author{
Johannes Blümlein \\ Deutsches Elektronen-Synchrotron, DESY, \\ Platanenallee 6, D-15738 Zeuthen, Germany
}

December 3, 2012

\begin{abstract}
The nucleon structure functions probed in deep-inelastic scattering at large virtualities form an important tool to test Quantum Chromdynamics (QCD) through precision measurements of the strong coupling constant $\alpha_{s}\left(M_{Z}^{2}\right)$ and the different parton distribution functions. The exact knowledge of these quantities is also of importance for all precision measurements at hadron colliders. During the last two decades very significant progress has been made in performing precision calculations. We review the theoretical status reached for both unpolarized and polarized lepton-hadron scattering based on perturbative QCD.
\end{abstract}

\section{Introduction}

Matter consists of regular structures at microscopic distances, which exhibit themselves at the crystalline, molecular, and atomic levels [1]. The discovery of $\alpha, \beta$ and $\gamma$ radioactivity [2] provided new natural probes beyond the visible spectrum of light and $X$-rays to resolve even smaller structures of matter. In $1911 \mathrm{E}$. Rutherford discovered the atomic nucleus of a size much smaller than that of atoms through scattering of $\alpha$-particles at gold [3]. Herewith the picture of matter at small distances changed dramatically rising the question for further sub-structures. The composite nature of nuclei could be explained after Chadwick's [4] discovery of the neutron and Yukawa's model for nuclear forces [5]. Another important discovery was made by Frisch and Stern in 1933 measuring the anomalous magnetic moment of the proton with a different value from that of point-particles, like electrons [6]. Later in 1939 Alvarez and Bloch measured the anomalous magnetic moment of the neutron [7], both of which constituted first evidence on the compositeness of nucleons. The current values of the nucleon magnetic moments are [8]

$$
\mu_{p}=2.792847356 \pm 0.000000023 \mu_{N}, \quad \mu_{n}=-1.9130427 \pm 0.0000005 \mu_{N}
$$

with $\mu_{N}=e \hbar / 2 m_{p}$ the nuclear magneton.

During the 1950ies the Hofstadter experiments [9] operated at virtualities being large enough to reveal the charge distribution inside nucleons, which is illustrated in Figure1. A positive core distribution and tail are found both for the proton and neutron, with a positive vector cloud in case of the proton and a negative one for the neutron, pointing to first details of the nucleon sub-structure. However, the specific nature of these distributions remained yet unexplained. 


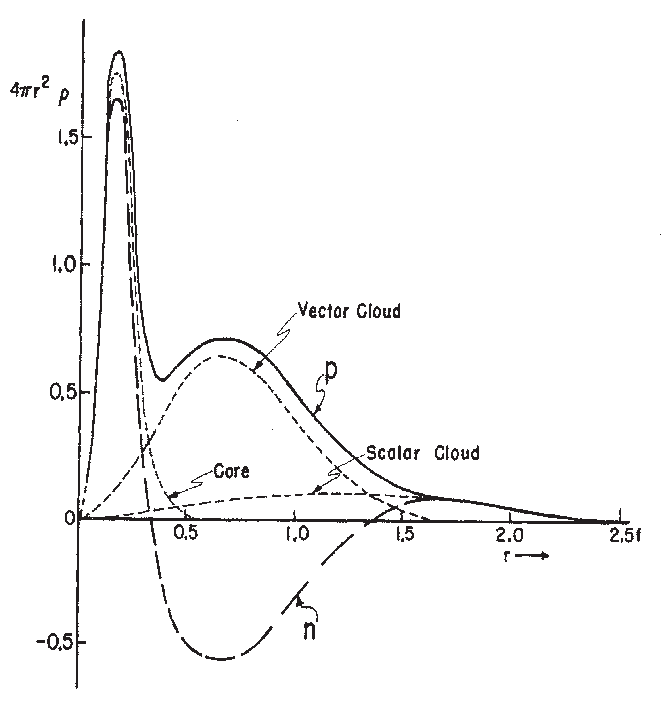

Figure 1: Charge distribution for the proton and the neutron implied by the form factors of Ref. [10], Figure 2(b); from [10], ㄷ(1961) by the American Physical Society.

In 1964 Gell-Mann [11] and Zweig [12] proposed the quarks 11 as building blocks of hadrons to catalog the plethora of observed mesons and baryons. During the late 1960ies the MIT-SLAC experiments [13 19] measured deep-inelastic electron-nucleon scattering at the Stanford Linear Accelerator at much shorter distances and beyond the resonance region. The important finding of these experiments were scaling and the observation that the longitudinal structure function is small, Figure 2, confirming a prediction by Callan and Gross [20] for scattering off spin 1/2 particles. The scaling behaviour of structure functions had been predicted by Bjorken using current algebra methods [21]. These new observations led Feynman to the parton model [22,23] of point-like fermionic constituents of the nucleons which react at high virtualities with the exchanged gauge bosons in the deep-inelastic process directly.
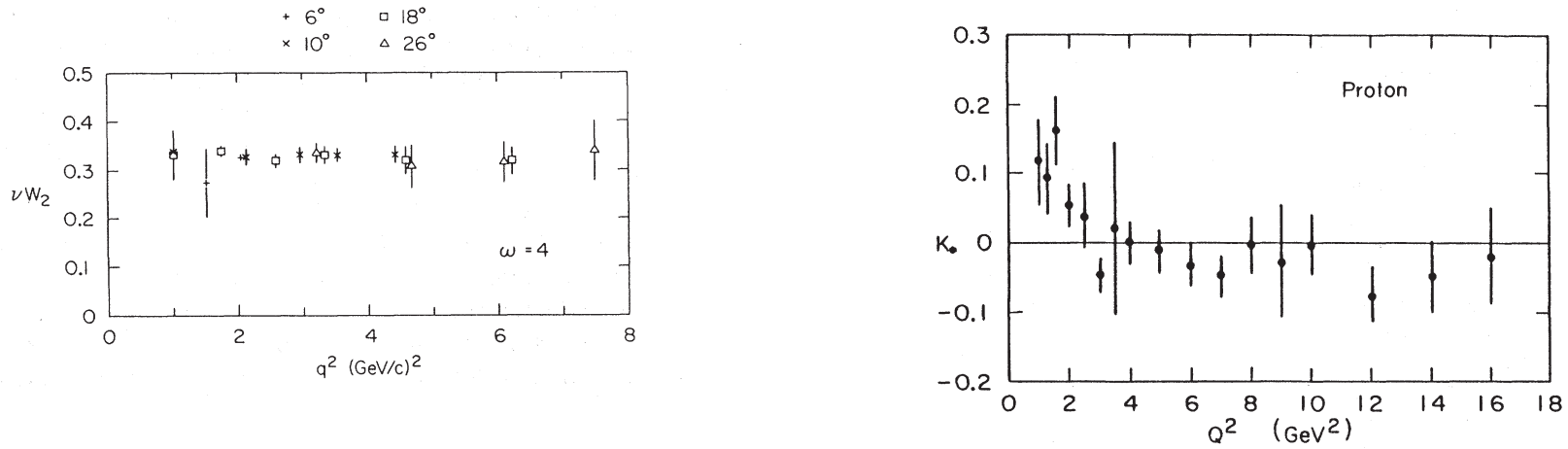

Figure 2: Left: An early observation of scaling: $\nu W_{2}$ for the proton as a function of $-q^{2}$ for $W>2 \mathrm{GeV}$, at $x=1 / \omega=0.25$; Right: The Callan-Gross relation: $K_{0}=F_{2} /\left(2 x F_{1}\right)-1$ vs $-q^{2}$. These results established the spin of the partons as $1 / 2$; from [18], ()(1991) by the American Physical Society.

Deep-inelastic scattering off constituent quarks has been discussed as early as 1967 [24] in connection to data of that time [25]. After the discovery of scaling at SLAC also data taken in other experiments were analyzed for this behaviour. One example concerns data taken at DESY at lower values of $\left|q^{2}\right|[26]$, cf. Figure 3, presented using the Rittenberg-Rubinstein variable $\omega_{W}$.

\footnotetext{
${ }^{1}$ G. Zweig named the hadron constituents aces.
} 


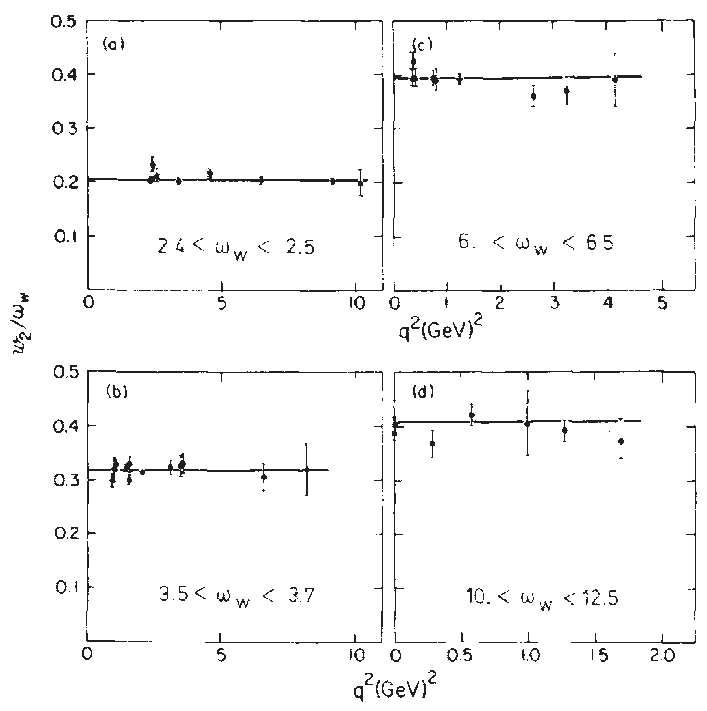

Figure 3: The function $\nu W_{2}$ plotted vs $\left|q^{2}\right|$ assuming $R=\left|q^{2}\right| / \nu^{2}$ for different fixed $\omega_{W}=\left(1 / x+M_{N}^{2} /\left|q^{2}\right|\right) /(1+$ $\left.0.2 /\left|q^{2}\right|\right)$; from [26] ()(1972) by Elsevier Science.

The parton model introduced a new level of compositeness for fermions being confined inside hadrons and related to the strong interactions. The final quantum field theory of the strong interactions developed over a series of years. Already in 1965 Nambu [27] proposed a Yang-Mills [28] SU(3) gauge theory for the strong interactions, based on a three-valued charge degree of freedom [29]. Before a symmetry was introduced using para-statistics [30] which later became color. At this time it was unknown whether Yang-Mills theories could be renormalized. The formalism by Faddeev and Popov [31] needed for their quantization in covariant gauges has been found two years later only. The renormalization of massless Yang-Mills theories was proven by 't Hooft in 1971 [32] and Quantum Chromodynamics (QCD) as the theory of strong interactions was proposed by Fritzsch and Gell-Mann in 1972 [33] and Fritzsch, Gell-Mann and Leutwyler [34]. In 1973 Gross, Wilczek [35] and Politzer [36] studied the running of the strong coupling constant of color octet Yang-Mills theory with color triplet quarks and found asymptotic freedom, see also [37,38. The Lagrangian of QCD, referring to the covariant $R_{\xi}$-gauges, is given by [39]

$$
\mathcal{L}_{\mathrm{QCD}}=\sum_{q} \bar{\psi}_{q, j}(x)\left[i \not D^{j k}-m_{q}\right] \psi_{q, k}(x)-\frac{1}{4} F_{a}^{\mu \nu}(x) F_{\mu \nu}^{a}(x)-\frac{1}{2 \xi}\left(\partial_{\mu} A_{a}^{\mu}(x)\right)^{2}+\partial_{\mu} \chi_{\mu}^{a}(x) D^{a b, \mu} \chi_{b}(x)
$$

where $\psi_{q}(x)$ denotes the quark fields, $A_{a}^{\mu}(x)$ the gluon fields, $F_{a}^{\mu \nu}=\partial^{\mu} A_{a}^{\nu}-\partial^{\nu} A_{a}^{\mu}+g f_{a b c} A^{b, \mu} A^{c, \nu}$ the field strength tensor, $f_{a b c}$ the structure constants of $S U(3)_{c}$, the gauge group of QCD, $\xi \in \mathbb{R}$ the gauge parameter, $\chi_{a}(x)$ the ghost field, and the covariant derivatives $D_{\mu}^{a b}=\delta^{a b} \partial_{\mu}-g f^{a b c} A_{c, \mu}(x), \not D^{j k}=$ $\gamma_{\mu}\left[\delta_{j k} \partial^{\mu}-i g A_{a}^{\mu} t_{j k}^{a}\right]$, with $t^{a}$ the generators of $S U(3)_{c}$. Based on this, perturbative calculations in Quantum Chromodynamics can be performed at large virtualities. Due to their high complexity these calculations are usually being performed using computer algebra programs, a first dedicated of which was SCHOONSCHIP by M. Veltman [40].

At short distances, resp. large scales of the momentum transfer, the nucleon structure functions $F_{i}\left(x, Q^{2}\right)$ obey the light-cone expansion [41 44] through which they are represented in terms of Wilson coefficients $C_{i}^{k}$ and non-perturbative operator matrix elements (OMEs) $f_{k}$ of local operators, characterized by their twist [45]. At leading twist the functions $f_{k}$ are the parton densities and at higher twist they correspond to parton correlation functions. In this separation an arbitrary scale $\mu_{F}^{2}$, the factorization scale, emerges. Applying the renormalization group equations [46 49] to the structure functions one obtains separate evolution equations for the Wilson coefficients and operator matrix elements which are usually of matrix-type due to the mixing of different operators. The solution of these 
evolution equations exhibits scaling violations 2 , implied by the renormalization group equations, and the running of the strong coupling constant and finite quark masses.

During the last 40 years the deep-inelastic structure functions both in neutrino and charged leptonnucleon scattering were measured at a steadily increasing precision and allow at present for important QCD precision tests at the per cent level. The strong coupling constant $\alpha_{s}\left(M_{Z}^{2}\right)$ can be measured at an accuracy of $\sim 1 \%$ from the scaling violations of the deep-inelastic structure functions. Very accurate extractions of the twist-2 parton densities are possible, which are an essential ingredient for the physics at hadron colliders as Tevatron and the LHC, and are thus instrumental in the search for new elementary particles.

At the theoretical side, the deep-inelastic structure functions are best described at the level of the twist-2 contributions. The QCD coupling constant is known to 4-loop order [53, 54]. The anomalous dimensions and Wilson coefficients for the unpolarized massless case were calculated to 3-loop order [55 61]. There are first results at 4-loop order [62 64. Heavy quark corrections are known to 2-loop order [65, 66] and for larger scales a series of Mellin moments has been calculated to 3-loop order 67]. In the polarized case the level of 2-loop corrections has been reached for massless and massive corrections [68 72], with first results at 3-loop order [73]. The present massless and massive 3- and 4loop calculations require powerful computer algebra systems and packages like FORM [74], MATAD [75], and Sigma [76], cf. also [77], to perform these voluminous calculations which amount even in parallel form to several CPU years and request $O(100$ Gbyte) RAM at multi-processor systems. QED corrections to the deep-inelastic scattering cross sections are large in certain kinematic regions and have to be known at the accuracy defined by the measured data. The region of small values of $x$ or large hadronic masses at a given virtuality requires special attention because of potentially large perturbative corrections. In the large $x$ region target mass effects and higher twist corrections contribute. For large values of $x$ higher order resummations are important. In the polarized case the structure function $g_{2}\left(x, Q^{2}\right)$ provides an inclusive observable at which twist-3 contributions can be studied. Structure functions and parton distributions obey a series of sum rules, to which also QCD corrections are available and one may perform QCD tests using these relations. The nucleon spin problem [78] rouse the question of also angular momentum contributions. These can be accessed in deeply-virtual Compton scattering and similar reactions [79] as has been shown in [80].

In this article we review the present theoretical understanding of the deep-inelastic process, mainly in the region of large scales. In Section 2 the scattering cross sections are described and a brief survey on the present deep inelastic data is given. Section 3 deals with the light-cone expansion and in Section 4 the parton model is described. The renormalization of deep-inelastic structure functions in the twist-2 approximation is discussed in Section 5. Related to this, a survey on the perturbative expansion of the strong coupling constant $\alpha_{s}\left(Q^{2}\right)$, the anomalous dimensions, resp. splitting functions, and the massless Wilson coefficients are given in Section 6-8. In Section 9 we discuss QED and electro-weak corrections to deep inelastic scattering. The heavy quark corrections are described in Section 10. Section 11 deals with the target mass corrections. The solution of the evolution equations is outlined in Section 12 . Here we also discuss the results of different NNLO QCD analyses and the status of the determination of $\alpha_{s}\left(M_{Z}^{2}\right)$ from deep-inelastic data. In Section 13 a series of aspects of small- $x$ resummations are considered. Resummations in the region of large values of $x$ are discussed in Section 14. Major sum rules and integral relations for deep-inelastic structure functions are summarized in Section 15. Higher twist corrections and aspects of nuclear parton distributions are considered in Sections 16 and 17 . In this review we will fully concentrate on firmly established results on the basis of perturbative Quantum Chromodynamics and will not discuss other approaches of a more phenomenological character, including other assumptions.

\footnotetext{
${ }^{2}$ Scaling violations emerge naturally through radiative corrections in all renormalizable field theories. Drell and collaborators at the end of the 1960ies were seeking for scaling describing structure functions by fermion-meson interactions with loop corrections and found scaling violations in general [50, 51], see also [52].
} 


\section{The deep-inelastic process}

The deep-inelastic process, at Born level, can be illustrated by the diagram shown in Figure 4 . A lepton $l=e^{ \pm} \mu^{ \pm}, \nu_{i}\left(\bar{\nu}_{i}\right)$ with momentum $k_{1}$ scatters off a nucleon $N$ exchanging an electro-weak gauge boson $V=\gamma, Z^{0}, W^{ \pm}$to a lepton $l^{\prime}$ with momentum $k_{2}=k_{1}-q$ and an ensemble of hadrons $X$ in an effective $2 \rightarrow 2$ process $k_{1}+p_{1} \rightarrow k_{2}+p_{2}$. Here $p_{1}$ is the momentum of the incoming nucleon and $p_{2}$ the momentum of the outgoing hadrons.

In many deep-inelastic scattering experiments the kinematic variables can be measured only from a sub-set of the momenta of the external particles. This applies in particular to the momentum of outgoing neutrinos but also for the momentum $p_{2}$ in fixed target experiments. As will be shown later in Section 9 the size of the radiative corrections strongly depends on the choice of the kinematic variables. For the measurement at HERA a wide variety of sets of kinematic variables was designed to allow for cross checks and to limit the size of QED radiative corrections.

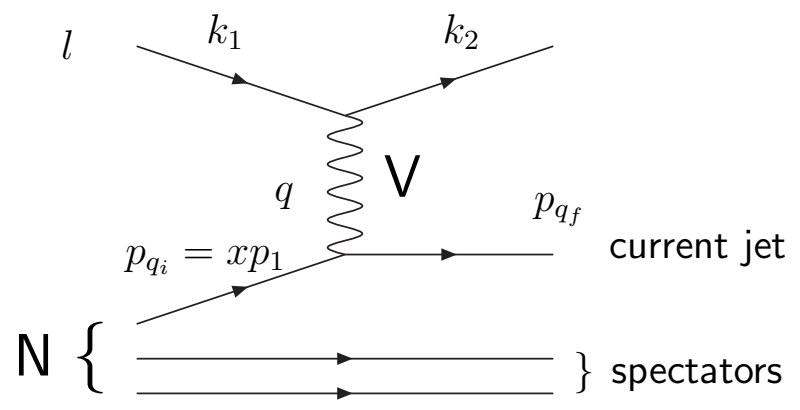

Figure 4: Diagram describing deep inelastic $l N$ scattering.

At Born level the unpolarized scattering cross section depends on the virtuality of the exchanged gauge boson $V, q^{2}=-Q^{2}$ and the inelasticity $y$. At a given cms energy $s=\left(k_{1}+p_{1}\right)^{2}$ these quantities define the Bjorken variable $x$,

$$
\begin{aligned}
& y=\frac{p_{1} \cdot\left(k_{1}-k_{2}\right)}{p_{1} \cdot k_{1}} \\
& x=\frac{Q^{2}}{s y} .
\end{aligned}
$$

The different sets of variables are :

i) Leptonic variables

ii) Hadronic variables [81]

iii) Jacquet-Blondel variables [82]

iv) Mixed variables [81]

v) Double angle method 83]

vi) $\theta y$ method 84

vii) $\Sigma$ method 85]

viii) $e \Sigma$ method 85 .

$$
\begin{aligned}
& q \equiv q_{l}=k_{2}-k_{1}, \quad y_{l}=p_{1} \cdot\left(k_{1}-k_{2}\right) / p_{1} \cdot k_{1} \\
& q \equiv q_{h}=p_{2}-p_{1}, \quad y_{l}=p_{1} \cdot\left(p_{2}-p_{1}\right) / p_{1} \cdot k_{1} \\
& Q_{J B}^{2}=\left(\vec{p}_{2, \perp}\right)^{2} /\left(1-y_{J B}\right), \quad y_{J B}=\Sigma /\left(2 E\left(k_{1}\right)\right) \\
& \Sigma=\sum_{h}\left(E_{h}-p_{h, z}\right) \\
& q=q_{l}, y_{m}=y_{J B} \\
& Q_{D A}^{2}=\frac{4 E\left(k_{2}\right)^{2} \cos ^{2}\left(\theta\left(k_{2}\right) / 2\right)}{\sin ^{2}\left(\theta\left(k_{2}\right) / 2\right)+\sin \left(\theta\left(k_{2}\right) / 2\right) \cos \left(\theta\left(k_{2}\right) / 2\right) \tan \left(\theta\left(p_{2}\right) / 2\right)}, \\
& y_{D A}=1-\frac{\sin \left(\theta\left(k_{2}\right) / 2\right)}{\sin \left(\theta\left(k_{2}\right) / 2\right)+\cos \left(\theta\left(k_{2}\right) / 2\right) \tan \left(\theta\left(p_{2}\right) / 2\right)}, \\
& Q_{\theta y}^{2}=4 E\left(k_{2}\right)^{2}\left(1-y_{J B}\right) \frac{1+\cos \left(\theta\left(k_{2}\right)\right)}{1-\cos \left(\theta\left(k_{2}\right)\right)}, y_{\theta y}=y_{J B} \\
& Q_{\Sigma}^{2}=\frac{\left(\vec{k}_{2, \perp}\right)^{2}}{1-y_{\Sigma}}, \quad y_{\Sigma}=\frac{\Sigma}{\Sigma+E\left(k_{2}\right)\left[1-\cos \left(\theta\left(k_{2}\right)\right)\right]} \\
& Q_{e \Sigma}^{2}=Q_{l}^{2}, \quad y_{e \Sigma}=\frac{Q_{l}^{2}}{s x_{\Sigma}}
\end{aligned}
$$


Here $E\left(l_{i}\right)$ and $\theta\left(l_{i}\right)$ are measured in the detector's rest frame. The invariant mass $W$ of the final state hadrons is given by

$$
W^{2}=p_{2}^{2}=\left(q+p_{1}\right)^{2}=M^{2}+2 q \cdot p_{1}-Q^{2}=M^{2}+Q^{2}(1-x) / x,
$$

and $M$ is the nucleon mass. The inclusive lepton-nucleon process is denoted as deep inelastic if $W>$ $2 \mathrm{GeV}$ and the values of $Q^{2}$ are sufficiently large, normally $Q^{2}>4 \mathrm{GeV}^{2}$. If $W \approx M$ the process is called (quasi-)elastic, allowing also for proton-neutron transitions. Here the scattering cross section is described by the (quasi-)elastic nucleon form-factors [86, 87]. The resonance region is characterized by $M<W \lesssim 2 \mathrm{GeV}$. Corresponding models for the scattering cross sections were given in [88].

The double differential scattering Born cross section off unpolarized nucleons read [87, 89, 90 ]

$$
\begin{aligned}
\frac{d^{2} \sigma_{\mathrm{NC}}^{l^{ \pm} N}}{d x d y} & =\frac{2 \pi \alpha^{2} s}{Q^{4}}\left\{\left[2(1-y)-2 x y \frac{M^{2}}{S}\right] \hat{F}_{2}\left(x, Q^{2}\right)+Y_{-} x \hat{F}_{3}\left(x, Q^{2}\right)+y^{2}\left(1-\frac{2 m_{l}^{2}}{Q^{2}}\right) 2 x \hat{F}_{1}\left(x, Q^{2}\right)\right\} \\
\frac{d^{2} \sigma_{\mathrm{NC}}^{\nu(\bar{\nu} N}}{d x d y} & =\frac{G_{F}^{2} s}{16 \pi}\left[\frac{M_{Z}^{2}}{Q^{2}+M_{Z}^{2}}\right]^{2}\left\{Y_{+} W_{2}^{\mathrm{NC}}\left(x, Q^{2}\right) \pm Y_{-} x W_{3}^{\mathrm{NC}}\left(x, Q^{2}\right)-y^{2} W_{L}^{\mathrm{NC}}\left(x, Q^{2}\right)\right\} \\
\frac{d^{2} \sigma_{\mathrm{CC}}}{d x d y} & =\frac{G_{F}^{2} s}{4 \pi}\left[\frac{M_{W}^{2}}{Q^{2}+M_{W}^{2}}\right]^{2}\left\{Y_{+} W_{2}^{\mathrm{CC}}\left(x, Q^{2}\right) \pm Y_{-} x W_{3}^{\mathrm{CC}}\left(x, Q^{2}\right)-y^{2} W_{L}^{\mathrm{CC}}\left(x, Q^{2}\right)\right\}
\end{aligned}
$$

where $\alpha$ and $G_{F}$ denote the fine-structure and Fermi constants, $M_{W, Z}$ and $m_{l}$ are the $W, Z$-boson and lepton masses and

$$
Y_{ \pm}=1 \pm(1-y)^{2}
$$

Three of the electro-weak parameters in (2.4 2.6) are independent. One choice is given by the set $\left(\alpha, M_{Z}, G_{F}\right)$. The others are expressed by corresponding relations, cf. [90]. In case of neutral current scattering the functions $\hat{F}_{i}$ are propagator-weighted structure functions, [90]:

$\hat{F}_{1,2}\left(x, Q^{2}\right)=F_{1,2}\left(x, Q^{2}\right)+2\left|Q_{e}\right|\left(v_{e}+\lambda a_{e}\right) \chi\left(Q^{2}\right) G_{1,2}\left(x, Q^{2}\right)+4\left(v_{e}^{2}+a_{e}^{2}+2 \lambda v_{e} a_{e}\right) \chi^{2}\left(Q^{2}\right) H_{1,2}\left(x, Q^{2}\right)$

$x \hat{F}_{3}\left(x, Q^{2}\right)=-2 \operatorname{sign}\left(Q_{e}\right)\left\{\left|Q_{e}\right|\left(a_{e}+\lambda v_{e}\right) \chi\left(Q^{2}\right) x G_{3}\left(x, Q^{2}\right)+\left[2 v_{e} a_{e}+\lambda\left(v_{e}^{2}+a_{e}^{2}\right)\right] \chi^{2}\left(Q^{2}\right) x H_{3}\left(x, Q^{2}\right)\right\}$,

with $Q_{e}=-1, \lambda=\operatorname{sign} \xi, v_{f}=1-4\left|Q_{f}\right| \sin ^{2} \theta_{w}^{\mathrm{eff}}, a_{f}=1, \chi\left(Q^{2}\right)=G_{F} M_{Z}^{2} Q^{2} /\left(\sqrt{2} 8 \pi \alpha\left(Q^{2}+M_{Z}^{2}\right)\right)$. $\theta_{w}^{\mathrm{eff}}$ denotes the effective weak mixing angle, $M_{Z}$ the $Z$-boson mass, and $\xi$ the lepton polarization. The structure function $2 x F_{1}$ can be expressed using

$$
2 x F_{1}\left(x, Q^{2}\right)=F_{2}\left(x, Q^{2}\right)-F_{L}\left(x, Q^{2}\right)
$$

and similar for $G_{1}, H_{1}, W_{1}^{ \pm}$. At this level the Callan-Gross relation [20] implies vanishing longitudinal structure functions, which take finite values due to QCD corrections and target mass effects, see Sections 8, 11.

The scattering cross sections (2.4 2.6) can be represented in the general form

$$
\sum_{i} \frac{d^{3} \sigma_{i}}{d x d y d \phi}=\sum_{i} P_{i}\left(s, Q^{2}\right) L_{i}^{\mu \nu} W_{\mu \nu, i}
$$

where $P_{i}\left(s, Q^{2}\right)$ denotes a propagator term and $L_{\mu \nu, i}$ and $W_{i}^{\mu \nu}$ are the respective contributions to the leptonic and hadronic tensor. Here we specified also the azimuthal angle of the final state lepton which 
emerges in the scattering cross section of polarized leptons off transversely polarized nucleons. The leptonic tensor can be calculated perturbatively. The hadronic tensor is given by

$$
W_{\mu \nu, i}=\frac{1}{4 \pi} \int d^{4} x e^{i q x}\left\langle P S\left|\left[J_{\mu}^{i_{1}}(x)^{\dagger}, J_{\nu}^{i_{2}}(0)\right]\right| P S\right\rangle .
$$

Here $S$ denotes the four-vector of the nucleon spin with $S \cdot P=0$ and the normalization $S^{2}=-M^{2}$. In the framework of the quark-parton model the currents $J_{\mu}^{j}$ are given by

$$
J_{\mu}^{j}(x)=\sum_{f, f^{\prime}} \bar{q}^{\prime}(x) \gamma_{\mu}\left(v_{q}^{j}+a_{q}^{j} \gamma_{5}\right) q(x) U_{f f^{\prime}},
$$

with $v_{q}^{j}$ and $a_{q}^{j}$ the vector and axial-vector couplings of the quarks. For charged current interactions $U_{f f^{\prime}}$ denotes the Cabibbo-Kobayashi-Maskawa matrix, whereas for neutral current interactions $U_{f f^{\prime}}=\delta_{f f^{\prime}}$. The hadronic tensor obeys the following properties [91, 92]

$$
\begin{aligned}
\text { Covariance : } & W_{\mu^{\prime} \nu^{\prime}}\left(q^{\prime}, P^{\prime}\right)=\Lambda_{\mu^{\prime}}^{\mu} \Lambda_{\nu^{\prime}}^{\nu} W_{\mu \nu}(q, P), \quad \Lambda \in L_{+}^{\uparrow} \\
\text { Hermiticity : } & W_{\mu \nu}(q, P)=W_{\nu \mu}^{*}(q, P) \\
\text { Spectrality : } & W_{\mu \nu}(q, P)=0, \quad-\frac{q^{2}}{2 P \cdot q}>1 \\
\text { Causality : } & \tilde{W}_{\mu \nu}(x, P)=\int d^{4} q e^{-i q x} W_{\mu \nu}(q, p) \\
\mathrm{T}-\text { invariance : } & W_{\mu \nu}(\bar{q}, \bar{P}, \bar{S})=\left[W^{\mu \nu}(q, P, S)\right]^{*},
\end{aligned}
$$

where $L_{+}^{\uparrow}$ is the orthochronous Lorentz group and $\bar{a}_{\mu}=a^{\mu}$. In general,

$$
\begin{aligned}
\text { Parity : } & W_{\mu \nu}(\bar{q}, \bar{P},-\bar{S})=W^{\mu \nu}(q, P, S) \\
\text { Symmetry : } & W_{\mu \nu}(q, P)=W_{\mu \nu}(-q, P) \\
\text { Current conservation : } & q_{\mu} W^{\mu \nu}=W^{\mu \nu} q_{\nu}=0 .
\end{aligned}
$$

are not obeyed. We construct the following hadronic tensor including the case of polarized targets, [93]:

$$
\begin{aligned}
W_{\mu \nu}= & \left(-g_{\mu \nu}+\frac{q_{\mu} q_{\nu}}{q^{2}}\right) F_{1}\left(x, Q^{2}\right)+\frac{\hat{P}_{\mu} \hat{P}_{\nu}}{P \cdot q} F_{2}\left(x, Q^{2}\right)-i \varepsilon_{\mu \nu \lambda \sigma} \frac{q^{\lambda} P^{\sigma}}{2 P \cdot q} F_{3}\left(x, Q^{2}\right) \\
& +\frac{q_{\mu} q_{\nu}}{P \cdot q} F_{4}\left(x, Q^{2}\right)+\frac{\left(p_{\mu} q_{\nu}+p_{\nu} q_{\mu}\right)}{2 P \cdot q} F_{5}\left(x, Q^{2}\right) \\
& +i \varepsilon_{\mu \nu \lambda \sigma} \frac{q^{\lambda} S^{\sigma}}{P \cdot q} g_{1}\left(x, Q^{2}\right)+i \varepsilon_{\mu \nu \lambda \sigma} \frac{q^{\lambda}\left(P \cdot q S^{\sigma}-S \cdot q P^{\sigma}\right)}{(P \cdot q)^{2}} g_{2}\left(x, Q^{2}\right) \\
& +\left[\frac{\hat{P}_{\mu} \hat{S}_{\nu}+\hat{S}_{\mu} \hat{P}_{\mu}}{2}-S \cdot q \frac{\hat{P}_{\mu} \hat{P}_{\nu}}{P \cdot q}\right] \frac{g_{3}\left(x, Q^{2}\right)}{P \cdot q} \\
& +S \cdot q \frac{\hat{P}_{\mu} \hat{P}_{\nu}}{(P \cdot q)^{2}} g_{4}\left(x, Q^{2}\right)+\left(-g_{\mu \nu}+\frac{q_{\mu} q_{\nu}}{q^{2}}\right) \frac{(S \cdot q)}{P \cdot q} g_{5}\left(x, Q^{2}\right), \\
+ & i \varepsilon_{\mu \nu \lambda \sigma} \frac{P_{\sigma} S_{\lambda}}{P \cdot q} g_{6}\left(x, Q^{2}\right)+S \cdot q \frac{q_{\mu} q_{\nu}}{P \cdot q)^{2}} g_{7}\left(x, Q^{2}\right) \\
& +\frac{\left(p_{\mu} q_{\nu}+q_{\mu} p_{\nu}\right) S \cdot q}{2(P \cdot q)^{2}} g_{8}\left(x, Q^{2}\right)+\frac{S_{\mu} q_{\nu}+S_{\nu} q_{\mu}}{2 P \cdot q} g_{9}\left(x, Q^{2}\right),
\end{aligned}
$$

with

$$
\hat{P}_{\mu}=P_{\mu}-\frac{P \cdot q}{q^{2}} q_{\mu}, \quad \hat{S}_{\mu}=S_{\mu}-\frac{S \cdot q}{q^{2}} q_{\mu}
$$


The choice of the polarized structure functions beyond $g_{1,2}$ is not unique in the literature, cf. [94]. For the functions $g_{3,4,5}$ we used the convention of [94], and for the further structure functions that of [95, 96]. The spin-vector in the longitudinal and transversal case is defined by

$$
\begin{aligned}
& S_{L}=(0,0,0, M) \\
& S_{T}=M(0, \cos \alpha, \sin \alpha, 0) .
\end{aligned}
$$

For the purely polarized nucleon contributions one obtains the following differential scattering cross sections, 93.

$$
\begin{aligned}
\frac{d^{2} \sigma\left(\lambda, \pm S_{L}\right)}{d x d y} & = \pm 2 \pi s \frac{\alpha^{2}}{Q^{4}} \sum_{i} C_{i} \eta_{i}\left(Q^{2}\right) \\
& \times\left[-2 \lambda y\left(2-y-\frac{2 x y M^{2}}{S}\right) x g_{1}^{i}+8 \lambda \frac{y x^{2} M^{2}}{S} g_{2}^{i}+\frac{4 x M^{2}}{s}\left(1-y-\frac{x y M^{2}}{s}\right) g_{3}^{i}\right. \\
& -2\left(1+\frac{2 x M^{2}}{s}\right)\left(1-y-\frac{x y M^{2}}{s}\right) g_{4}^{i}-2 x y^{2}\left(1+\frac{2 x M^{2}}{s}\right) g_{5}^{i} \\
& \left.+4 \lambda \frac{x y M^{2}}{s} g_{6}^{i}-2\left(1-y-\frac{x y M^{2}}{s}\right) g_{9}^{i}\right] \\
\frac{d^{3} \sigma\left(\lambda, \pm S_{T}\right)}{d x d y d \phi} & = \pm s \frac{\alpha^{2}}{Q^{4}} \sum_{i} C_{i} \eta_{i}\left(Q^{2}\right) \\
& \times 2 \sqrt{\frac{M^{2}}{s}} \sqrt{x y\left[1-y-\frac{x y M^{2}}{s}\right]} \cos (\alpha-\phi)\left[-2 \lambda y x g_{1}^{i}-4 \lambda x g_{2}^{i}\right. \\
& \left.-\frac{1}{y}\left(2-y-\frac{2 x y M^{2}}{s}\right) g_{3}^{i}+\frac{2}{y}\left(1-y-\frac{x y M^{2}}{s}\right) g_{4}^{i}+2 x y g_{5}^{i}-2 \lambda g_{6}^{i}-g_{9}^{i}\right]
\end{aligned}
$$

Here $\lambda$ denotes the lepton polarization and $C^{\gamma}=1, C^{\gamma Z}=g_{V}+\lambda g_{A}, g_{V}^{\gamma}=1, g_{A}^{\gamma}=0, g_{V}^{Z}=$ $\left(1-4 Q_{l} \sin ^{2} \theta_{W}^{\mathrm{eff}}\right) / 2, g_{A}^{Z}=-1 / 2, g_{V}^{W^{-}}=1, g_{A}^{W^{-}}=-1, C^{Z}=\left(g_{V}+\lambda g_{A}\right)^{2}, C^{W^{ \pm}}=(1 \pm \lambda), \eta^{|\gamma|^{2}}=$ $1, \eta^{\gamma Z}=G_{F} Q^{2} /(2 \sqrt{2} \pi \alpha)\left(M_{Z}^{2} /\left(Q^{2}+M_{Z}^{2}\right), \eta^{|Z|^{2}}=\left(\eta^{\gamma Z}\right)^{2}, \eta^{\left|W^{ \pm}\right|^{2}}=\left[G_{F} Q^{2} /(4 \pi \alpha)\left(M_{W}^{2} /\left(Q^{2}+M_{W}^{2}\right)\right]^{2} .3\right.\right.$ The structure functions $g_{7}$ to $g_{9}$ do not contribute in case of vanishing lepton masses and the structure functions $F_{4,5}$ are related to $F_{1,2,3}$.

The individual structure functions can be measured form the scattering cross sections (2.4] 2.6][2.24, 2.25) applying the QED and electro-weak radiative corrections to the data, cf. Section 9. Current data analyses in the unpolarized case refer to the structure function data on $F_{2}$ and charged current scattering cross sections for proton and deuteron targets taken at SLAC [98, 99], from BCDMS [100, 101], NMC [102, 103] at CERN, and HERA [104]. Surveys on earlier neutrino and charged lepton deepinelastic data were given in [105].

Moreover, there are data on the longitudinal structure function $F_{L}\left(x, Q^{2}\right)$ [99, 100, 103, 106, 109]. To separate the contributions due to the different sea-quark flavors Drell-Yan data [110] and di-muon data [111] are used. In some analyses [112,113 also other sets of inclusive and semi-inclusive deepinelastic scattering data, including data on $F_{2}^{Q \bar{Q}}\left(x, Q^{2}\right), Q=c, b$, data from E665, CHORUS, and Tevatron data on weak boson production [114 are used. Various PDF-fitting groups use also the Tevatron jet data [115] in their analysis.

${ }^{3} \mathrm{~A}$ factor of $1 / 2$ has to be corrected for $\left.\eta^{\mid W^{ \pm}}\right|^{2}$ in [93, 94, cf. [97]. 

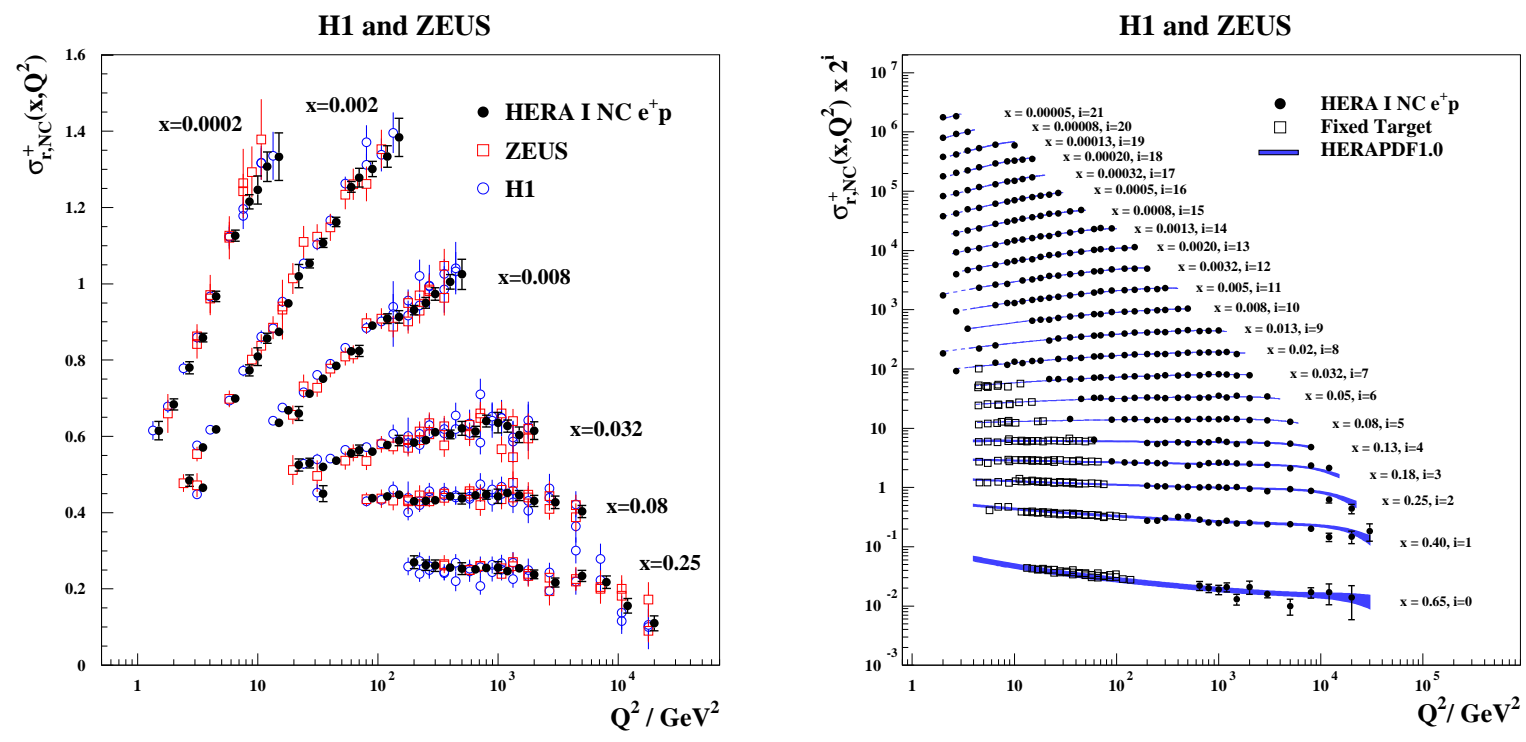

Figure 5: Left: HERA combined NC $e^{+} p$ reduced cross section as a function of $Q^{2}$ for six $x$-bins compared to the separate $\mathrm{H} 1$ and ZEUS data input to the averaging procedure. The error bars indicate the total experimental uncertainty. The individual measurements are displaced horizontally for better visibility; Right : The combined data with the HERAPDF1.0 fit [116] is superimposed. The bands represent the total uncertainty of the fit. Dashed lines are shown for $Q^{2}$ values not included in the QCD analysis; from [104] ${ }^{\complement}(2009)$ Springer Verlag.

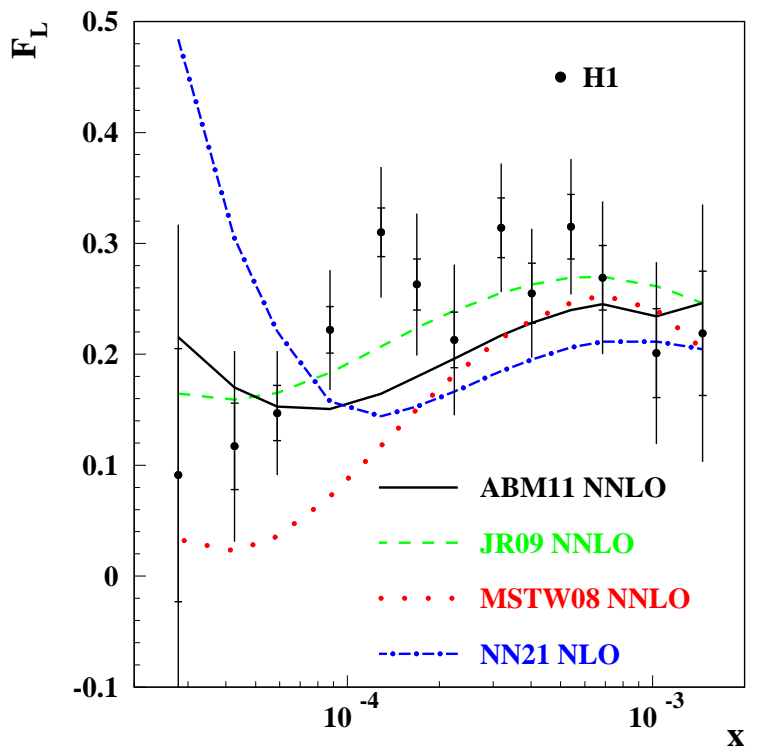

Figure 6: The data on $F_{L}$ versus $x$ obtained by the $\mathrm{H} 1$ collaboration [109] confronted with the 3-flavor scheme NNLO predictions based on the different parton distributions functions (PDFs). Solid line: ABM11 [117], dashes: JR09 [118], dots: MSTW [112]). The NLO predictions based on the 3-flavor NN21 PDFs [119] are given for comparison (dashed dots). The value of $Q^{2}$ for the data points and the curves in the plot rises with $x$ in the range of $1.5 \div 45 \mathrm{GeV}^{2}$; from [117].

In the polarized case the data stem from SLAC [120 124] EMC, SMC, COMPASS [78, 125] 128] at CERN, CLAS [130 132, and HERMES [133, 134] at HERA, taken at $p, d$, and $n\left({ }^{3} \mathrm{He}\right)$ targets on the 
polarization asymmetry $A_{1}$, the ratio $g_{1} / F_{1}$ or for the structure function $g_{1}$. Data on the structure function $g_{2}\left(x, Q^{2}\right)$ were taken by the SLAC experiments, SMC and HERMES [122, 135].

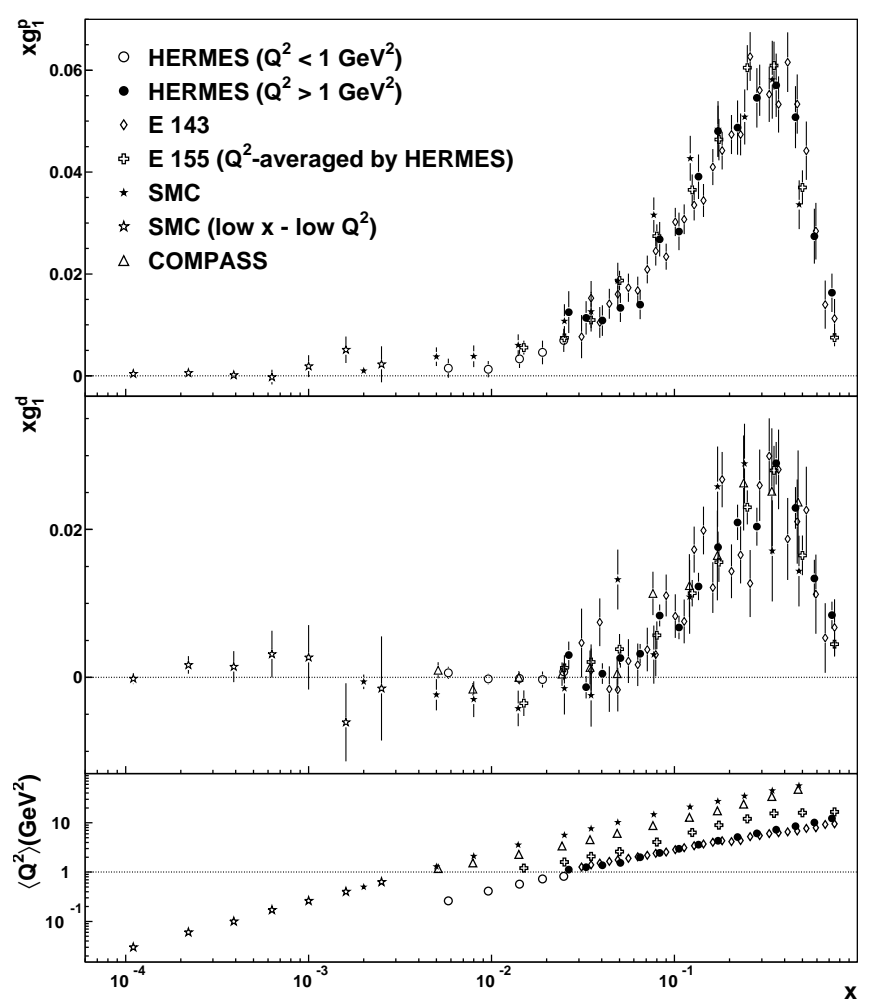

Figure 7: HERMES results on $x g_{1}^{p}$ and $x g_{1}^{d}$ vs $x$, shown on separate panels, compared to data from SMC [126], E143 [122], E155 [123,124] and COMPASS [129]. The error bars represent the sum in quadrature of statistical and systematic uncertainties. The HERMES data points shown are statistically correlated by unfolding QED radiative and detector smearing effects; the statistical uncertainties shown are obtained from only the diagonal elements of the covariance matrix. The E143 and E155 data points are correlated due to the method for correcting for QED radiation. For the HERMES data the closed (open) symbols represent values derived by selecting events with $Q^{2}>1 \mathrm{GeV}^{2}\left(Q^{2}<1 \mathrm{GeV}^{2}\right)$; from [134] ${ }^{\complement}(2007)$ by the American Physical Society.

The current precision in the measurement of the unpolarized structure function $F_{2}\left(x, Q^{2}\right)$ is illustrated in Figure 5 showing the combined HERA data together with the world fixed target data. Also the individual measurements of $\mathrm{H} 1$ and ZEUS are compared. The present experimental errors reach the $1 \%$ level and the scaling violations of $F_{2}\left(x, Q^{2}\right)$ are clearly visible over a wide range in $Q^{2}$.

In Figure 6 recent measurements of the structure function $F_{L}\left(x, Q^{2}\right)$ at low values of $x$ by the H1 experiment are shown together with predictions by different PDF-fits. The structure function $F_{L}\left(x, Q^{2}\right)$ can only be measured changing the beam energy in given bins of $\left(x, Q^{2}\right)$ to separate it from $F_{2}\left(x, Q^{2}\right)$ via the $y$-dependence of the scattering cross section. A precise measurement requires high luminosities and can be expected at the EIC, and at even smaller values of $x$ at a future high energy ep collider.

Finally, we illustrate the current precision of the measurement of the polarized structure function $g_{1}\left(x, Q^{2}\right)$ at $p$ and $d$ targets in Figure 7 . If compared to the structure function $F_{2}\left(x, Q^{2}\right)$ the experimental errors are larger since $g_{1}\left(x, Q^{2}\right)$ is measured from a polarization asymmetry. The range in $Q^{2}$ of these measurements is smaller than in case of $F_{2}\left(x, Q^{2}\right)$ since all measurements were performed in fixed target experiments.

In Figure 8 the kinematic region which has been probed by the different fixed target experiments at CERN, SLAC and the HERA experiments is illustrated. For virtualties $Q^{2} \geq 4 \mathrm{GeV}^{2}$ values of $x \simeq 4 \cdot 10^{-4}$ are reached. The highest values of $Q^{2}$ reached are $\sim 20.000 \mathrm{GeV}^{2}$. There is a proposal for 
an ep experiment in the LHC ring operating at $E_{p}=7 \mathrm{TeV}$ and $E_{e}=140 \mathrm{GeV}$, which would extend the present $x$ and $Q^{2}$ ranges by about 1.5 orders of magnitude [136].

The kinematic region for polarized data ranges for $0.005<x<0.75$ and $1 \lesssim Q^{2} \lesssim 70 \mathrm{GeV}^{2}$ with a kinematic correlation between these two variables. At a future facility like the Electron-Ion Collider EIC [137, 138] this region will be extended significantly. Moreover, the high luminosity available will allow precision measurements also for polarized scattering.

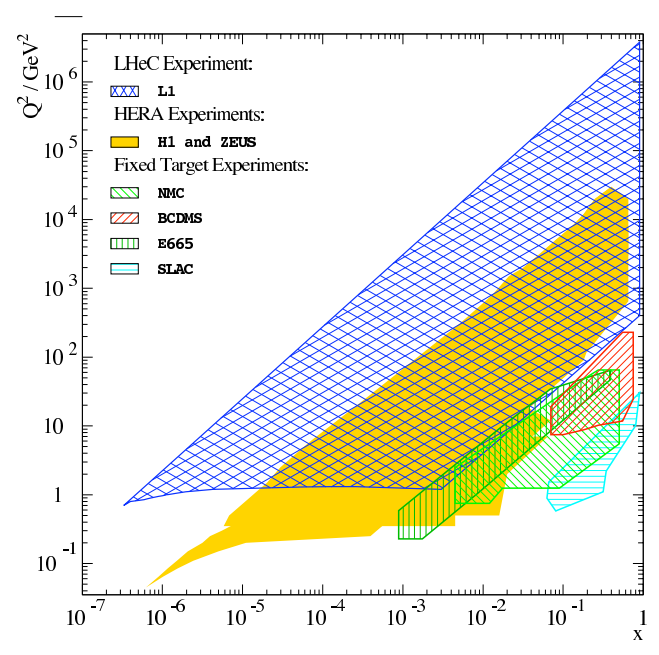

Figure 8: The kinematic range in $x$ and $Q^{2}$ probed by the deep-inelastic scattering experiments at HERA, CERN, and SLAC. The region accessible to a possible future $e^{ \pm} p$ experiment with $E_{p}=7 \mathrm{TeV}$ and $E_{e}=140 \mathrm{GeV}$ is also shown; by courtesy of M. Klein and E. Lobodzinska.

\section{The Light-Cone Expansion}

We consider the hadronic tensor (2.12) and limit the discussion for brevity to the case of pure photon exchange. It is given by the absorptive part of the forward Compton-amplitude $T_{\mu \nu}$

$$
W_{\mu \nu}(P, q)=\frac{1}{\pi} \operatorname{Im} T_{\mu \nu}(P, q)
$$

with

$$
\begin{aligned}
T_{\mu \nu}(P, q) & =i \int d^{4} \xi e^{i q \xi}\left\langle P\left|\mathrm{~T} J_{\mu}(\xi) J_{\nu}(0)\right| P\right\rangle \\
& =\frac{1}{2 x}\left(g_{\mu \nu}+\frac{q_{\mu} q_{\nu}}{Q^{2}}\right) T_{1}\left(x, Q^{2}\right)+\frac{2 x}{Q^{2}}\left(P_{\mu} P_{\nu}+\frac{P_{\mu} q_{\nu}+P_{\nu} q_{\mu}}{2 x}-\frac{Q^{2}}{4 x^{2}} g_{\mu \nu}\right) T_{2}\left(x, Q^{2}\right) .
\end{aligned}
$$

One may integrate (3.2), [44], which yields

$$
T_{\mu \nu}(P, q)=T_{\mu \nu}\left(q^{2}, \nu\right)=4 \pi \int_{-\infty}^{+\infty} d \xi_{0} \int_{0}^{\infty} d|\boldsymbol{\xi}||\boldsymbol{\xi}|\left[\frac{e^{i \nu \xi_{0}} \sin \left(\sqrt{\nu^{2}-q^{2}}|\boldsymbol{\xi}|\right)}{\sqrt{\nu^{2}-q^{2}}}\right] .
$$

For $\nu \rightarrow \infty$ the exponentials behave like

$$
e^{i \nu \xi_{0}} e^{ \pm i \sqrt{\nu^{2}-q^{2}}|\boldsymbol{\xi}|} \approx e^{i \nu\left(\xi_{0} \pm|\boldsymbol{\xi}|\right)} e^{ \pm i M|\boldsymbol{\xi}| x}
$$


Due to the Riemann-Lebesgue theorem [139] the dominant contributions to (3.4) come from

$$
\left|\xi_{0} \pm\right| \boldsymbol{\xi}|| \lesssim \frac{1}{\nu}, \quad|\boldsymbol{\xi}| \lesssim \frac{1}{M x},
$$

with $x$ the Bjorken variable. Thus

$$
\xi^{2}=\xi_{0}^{2}-\xi^{2} \lesssim \frac{1}{Q^{2}}
$$

and in the Bjorken limit $\nu, Q^{2} \rightarrow \infty$ the contributions very near to the light cone dominate.

At very short distances $\xi^{2} \approx 0$ the light-cone expansion of the time ordered product of currents has the following representation, [41 44] :

$$
\lim _{\xi^{2} \rightarrow 0} \mathrm{~T} J(x) J(0) \sim \sum_{i, N, \tau} \bar{C}_{i, \tau}^{N}\left(\xi^{2}, \mu^{2}\right) \xi_{\mu_{1}} \ldots \xi_{\mu_{N}} O_{i, \tau}^{\mu_{1}, \ldots, \mu_{N}}\left(0, \mu^{2}\right)
$$

Here we consider general currents and $O_{i, \tau}^{\mu_{1}, \ldots, \mu_{N}}$ denote local operators, which are finite as $\xi^{2} \rightarrow 0$, and $\bar{C}_{i, \tau}^{N}\left(\xi^{2}, \mu^{2}\right)$ are the corresponding Wilson coefficients. Let $D_{O}$ and $D_{J}$ be the canonical dimensions of the operators and currents and $N$ their global spin. The twist $\tau$ [45] of the operator is given by

$$
\tau=D_{O}-N
$$

The Wilson coefficients behave then like

$$
\bar{C}_{i, \tau}^{N}\left(\xi^{2}, \mu^{2}\right) \approx\left(\frac{1}{\xi^{2}}\right)^{-\tau / 2+d_{J}} .
$$

The local operators of lowest twist are given by

$$
\begin{aligned}
O_{q ; r ; \mu_{1}, \ldots, \mu_{n}}^{\mathrm{NS}}(0) & =i^{N-1} \mathbf{S}\left[\bar{\psi} \gamma_{P} \gamma_{\mu_{1}} D_{\mu_{2}} \ldots D_{\mu_{N}} \frac{\lambda_{r}}{2} \psi\right] \\
O_{q ; r ; \mu_{1}, \ldots, \mu_{n}}^{\mathrm{S}}(0) & =i^{N-1} \mathbf{S}\left[\bar{\psi} \gamma_{P} \gamma_{\mu_{1}} D_{\mu_{2}} \ldots D_{\mu_{N}} \psi\right] \\
O_{g ; r ; \mu_{1}, \ldots, \mu_{n}}^{\mathrm{S}}(0) & =2 i^{N-2} \mathbf{S S p}\left[E_{\mu_{1} \alpha}^{\beta \gamma} F_{\beta \gamma}^{a} D_{\mu_{2}} \ldots D_{\mu_{N-1}} F_{\mu_{N}}^{\alpha, a}\right] .
\end{aligned}
$$

Here the indices $q$ and $g$ refer to quark and gluon field operators, respectively, and $\lambda_{r}$ denotes the Gell-Mann matrix of the corresponding light flavor representation; $\psi$ is the quark field, $F_{\mu \nu}^{a}$ the gluonic field strength tensor in QCD, $D_{\mu}$ the covariant derivative, $\mathbf{S}$ the symmetry operator for all Lorentz indices and Sp the color-trace, where the index $a$ is the color index in the adjoint representation. In the unpolarized case $\gamma_{P}=\mathbf{1}$ and $E_{\mu_{1} \alpha}^{\beta \gamma}=\delta_{\mu_{1}}^{\beta} \delta_{\alpha}^{\gamma}$, while in the polarized case $\gamma_{P}=\gamma_{5}$ and $E_{\mu_{1} \alpha}^{\beta \gamma}=\frac{1}{2} \varepsilon_{\mu_{1} \alpha}^{\beta \gamma}$, where $\varepsilon_{\alpha_{1} \alpha_{2} \alpha_{3} \alpha_{4}}$ is the Levi-Civita symbol. The operators (3.11 3.13) still contain trace terms, which have to be subtracted. More generally than just by applying the symmetry operation a rigorous twist decomposition can be obtained applying the method of [140].

Let us consider the case of twist-2 operators in the following. We form the expectation value of the operator (3.8) between nucleon states and perform the Fourier transform of the Wilson coefficients through which

$$
T\left(x, Q^{2}\right)=\sum_{N} C^{N}\left(\frac{Q^{2}}{\mu^{2}}\right) A_{N}\left(\frac{\mu^{2}}{P^{2}}\right) \frac{1}{x^{N}}
$$

is obtained, where

$$
\begin{aligned}
\int d^{4} \xi e^{i \xi q} \xi_{\mu_{1}} \ldots \xi_{\mu_{N}} \bar{C}^{N}\left(\xi^{2}, \mu^{2}\right) & =\frac{1}{i}\left(\frac{2}{Q^{2}}\right)^{N} q_{\mu_{1}} \ldots q_{\mu_{N}} C^{N}\left(\frac{Q^{2}}{\mu^{2}}\right) \\
\left\langle P\left|O_{\mu_{1}, \ldots \mu_{N}}\left(0, \mu^{2}\right)\right| P\right\rangle & =P_{\mu_{1}} \ldots P_{\mu_{N}} A_{N}\left(\frac{\mu^{2}}{P^{2}}\right) .
\end{aligned}
$$


The sum in (3.14) runs over even or odd moments starting with a value $N_{0} \geq 0$ depending on the crossing relation of the currents, cf. e.g. 94. Here $\mu^{2}$ denotes the factorization scale. This scale is arbitrary and cancels between the Wilson coefficients and the operator matrix elements since the structure functions do not depend on it. Performing a contour integral in $x$ around the singularities of (3.14) one obtains the expressions for the structure functions in Mellin space:

$$
F_{2, L}\left(N, Q^{2}\right)=\mathbf{M}\left[F_{2, L}\left(x, Q^{2}\right)\right](N)=\sum_{i} C_{i}^{N}\left(\frac{Q^{2}}{\mu^{2}}\right) A_{i, N}\left(\frac{\mu^{2}}{P^{2}}\right)
$$

Eq. (3.17) shows the factorized form of the deep-inelastic structure function. The factorization theorems [141] state this form is remaining under higher order corrections.

Here the Mellin transformation [142] is given by

$$
\mathbf{M}[A(x)](N)=\int_{0}^{1} d x x^{N-1} A(x)
$$

with the Mellin-convolution

$$
\begin{aligned}
A(x) \otimes B(x) & =\int_{0}^{1} d x_{1} \int_{0}^{1} d x_{2} \delta\left(x-x_{1} x_{2}\right) A\left(x_{1}\right) B\left(x_{2}\right) \\
\mathbf{M}[A(x) \otimes B(x)](N) & =\mathbf{M}[A(x)](N) \mathbf{M}[B(x)](N) .
\end{aligned}
$$

The Wilson coefficients are perturbatively calculable and account for both the massless and massive quark contributions. The operator matrix elements $A_{i, N}$ are of non-perturbative nature. In case of twist2 they also denote the moments of the parton distributions for massless quarks and gluons, labeled by the index $i$,

$$
A_{i, N}\left(\frac{\mu^{2}}{P^{2}}\right) \equiv f_{i}\left(N, \mu^{2}\right)
$$

being discussed in the following Section.

\section{Parton Models}

The SLAC-MIT experiments [15, 16] found the strict correlation between the variables $\nu \equiv p \cdot q / M \nu$ and $Q^{2}$ for large enough values and at the fixed ratio

$$
\omega=\frac{2 M \nu}{Q^{2}}=\frac{1}{x}
$$

In the Bjorken limit [21] the structure functions are given by

$$
\begin{aligned}
\lim _{Q^{2}, \nu \rightarrow \infty} M W_{1}\left(Q^{2}, \nu\right) & \rightarrow F_{1}(x) \\
\lim _{Q^{2}, \nu \rightarrow \infty} \nu W_{2}\left(Q^{2}, \nu\right) & \rightarrow F_{2}(x) .
\end{aligned}
$$

In the (naive) parton model by Feynman [22,23] one assumes that the correlation (4.1) is exact. A nucleon at short distances is assumed to constitute of individual partons which are charged and move collinear to the nucleon momentum with momentum fractions $x_{i}, \sum_{i} x_{i}=1$. Let the parton which 
interacts with the virtual photon carry charge $e_{1}$ and momentum fraction $x_{1}$. The hadronic tensor is then given by

$$
\begin{aligned}
W_{\mu \nu} & =\frac{4 \pi}{M}\left[p_{\mu} p_{\nu} W_{2}-g_{\mu \nu} M^{2} W_{1}\right]=\int_{0}^{1} d x_{1} f\left(x_{1}\right) \frac{2 E}{2 E_{1}}|M|^{2} 2 \pi \delta\left(\left(x_{1} p+q\right)^{2}-m^{2}\right) \\
|M|^{2} & =\frac{1}{2} 4 e_{1}^{2}\left[p_{1 \mu} p_{1 \nu}^{\prime}+p_{1 \nu} p_{1 \mu}^{\prime}-g_{\mu \nu} p_{1} . q\right],
\end{aligned}
$$

with $p_{1}^{\prime}=p_{1}+q$ and $m$ the mass of the parton. $f\left(x_{1}\right)$ denotes the number density of the struck parton. The $\delta$-distribution, which incorporates the correlation (4.1) in (4.4), can be written as

$$
\delta\left(\left(x_{1} p+q\right)^{2}-m^{2}\right)=\delta\left(Q^{2}-2 x_{1} p \cdot q\right) \equiv \frac{1}{2 M \nu} \delta\left(x_{1}-x\right) .
$$

After performing the integral in (4.4) one obtains

$$
\nu W_{2}\left(Q^{2}, \nu\right)=\sum_{i} e_{i}^{2} x f_{i}(x), \quad 2 M W_{1}\left(Q^{2}, \nu\right)=\sum_{i} e_{i}^{2} f_{i}(x) .
$$

Here, we summed over all charged partons and anti-partons contained in the nucleon. In the Bjorken limit the structure functions are thus described by

$$
F_{1}(x)=\frac{1}{2} \sum_{i} e_{i}^{2} f_{i}(x), \quad F_{2}(x)=\sum_{i} e_{i}^{2} x f_{i}(x) .
$$

The parton distribution function $f_{i}(x)$ agrees with the Mellin inversion of Eq. (3.21). Furthermore, the Callan-Gross relation [20]

$$
F_{2}(x)=2 x F_{1}(x)
$$

holds. The above argument could have been also given assuming a micro-canonical ensemble in (4.4), [143.

In the parton model the unpolarized structure functions at the Born level in (2.4 2.6) are given by :

$$
\begin{array}{rlrl}
F_{2}\left(x, Q^{2}\right) & =x \sum_{q} Q_{q}^{2}\left[q\left(x, Q^{2}\right)+\bar{q}\left(x, Q^{2}\right)\right] & & |\gamma|^{2} \\
G_{2}\left(x, Q^{2}\right) & =x \sum_{q}\left|Q_{q}\right| v_{q}\left[q\left(x, Q^{2}\right)+\bar{q}\left(x, Q^{2}\right)\right] & & |\gamma Z| \\
H_{2}\left(x, Q^{2}\right) & =x \sum_{q} \frac{1}{4}\left(v_{q}^{2}+a_{q}^{2}\right)\left[q\left(x, Q^{2}\right)+\bar{q}\left(x, Q^{2}\right)\right] & & |Z|^{2} \\
x G_{3}\left(x, Q^{2}\right) & =x \sum_{q}\left|Q_{q}\right| a_{q}\left[q\left(x, Q^{2}\right)-\bar{q}\left(x, Q^{2}\right)\right] & & |\gamma Z| \\
x H_{3}\left(x, Q^{2}\right) & =x \sum_{q}^{\frac{1}{2}} v_{q} a_{q}\left[q\left(x, Q^{2}\right)-\bar{q}\left(x, Q^{2}\right)\right] & |Z|^{2} \\
W_{2}^{\mathrm{NC}}\left(x, Q^{2}\right) & =x \sum_{i}\left(v_{i}^{2}+a_{i}^{2}\right)\left[q_{i}\left(x, Q^{2}\right)+\bar{q}_{i}\left(x, Q^{2}\right)\right] & & |Z|^{2} \\
x W_{3}^{\mathrm{NC}}\left(x, Q^{2}\right) & =2 x \sum_{i} v_{i} a_{i}\left[q_{i}\left(x, Q^{2}\right)-\bar{q}_{i}\left(x, Q^{2}\right)\right] & |Z|^{2} \\
W_{2}^{\mathrm{CC},+}\left(x, Q^{2}\right) & =2 x \sum_{i}\left[d_{i}\left(x, Q^{2}\right)+\bar{u}_{i}\left(x, Q^{2}\right)\right] & \left|W^{+}\right|^{2} \\
W_{2}^{\mathrm{CC},-}\left(x, Q^{2}\right) & =2 x \sum_{i}\left[u_{i}\left(x, Q^{2}\right)+\bar{d}_{i}\left(x, Q^{2}\right)\right] & \left|W^{-}\right|^{2} \\
x W_{3}^{\mathrm{CC},+}\left(x, Q^{2}\right) & =2 x \sum_{i}\left[d_{i}\left(x, Q^{2}\right)-\bar{u}_{i}\left(x, Q^{2}\right)\right] & \left|W^{+}\right|^{2} \\
x W_{3}^{\mathrm{CC},-}\left(x, Q^{2}\right) & =2 x \sum_{i}\left[u_{i}\left(x, Q^{2}\right)-\bar{d}_{i}\left(x, Q^{2}\right)\right] & \left|W^{-}\right|^{2}
\end{array}
$$


Examples for the polarized structure functions (2.24,2.25) are [94, 144, 145]:

$$
\begin{aligned}
g_{1}^{|\gamma|^{2}}\left(x, Q^{2}\right) & =\sum_{q} Q_{q}^{2}\left[\Delta q\left(x, Q^{2}\right)+\Delta \bar{q}\left(x, Q^{2}\right)\right] \\
g_{1}^{|Z|^{2}}\left(x, Q^{2}\right) & =\sum_{q} \frac{1}{4}\left(v_{q}^{2}+a_{q}^{2}\right)\left[\Delta q\left(x, Q^{2}\right)+\Delta \bar{q}\left(x, Q^{2}\right)\right] \\
g_{5}^{|Z|^{2}}\left(x, Q^{2}\right) & =\sum_{q}^{q} \frac{1}{2} v_{q} a_{q}\left[\Delta q\left(x, Q^{2}\right)-\Delta \bar{q}\left(x, Q^{2}\right)\right], \text { etc. }
\end{aligned}
$$

Here $q_{i}\left(x, Q^{2}\right)$ and $\bar{q}_{i}\left(x, Q^{2}\right)$ denote the quark and antiquark distributions of the up $(u)$ and down $(d)$ type for four active flavors in the massless limit. $\Delta q_{i}\left(x, Q^{2}\right)$ and $\Delta \bar{q}_{i}\left(x, Q^{2}\right)$ are the corresponding polarized parton densities. $Q_{q}$ denotes the charge of the quark. Furthermore,

$$
g_{4}\left(x, Q^{2}\right)=2 x g_{5}\left(x, Q^{2}\right)
$$

holds. The remaining structure functions contain also twist-3 contributions [94,145, cf. Sections 15, 16.

The applicability of the parton picture rests on the comparison of two times [146]: $\tau_{\text {int }}-$ the interaction time of the virtual gauge boson with the hadron and $\tau_{\text {life }}$ - the life-time of individual partons. Let these times be measured in an infinite momentum frame, with $p$ the large longitudinal momentum. Applying old fashioned perturbation theory, cf. [147], they are given by

$$
\begin{aligned}
\tau_{\text {int }} & \sim \frac{1}{q_{0}}=\frac{4 p x}{Q^{2}(1-x)} \\
\tau_{\text {live }} & \sim \frac{1}{\sum_{i}\left(E_{i}-E\right)}=\frac{2 p}{\sum_{i}\left(k_{\perp, i}^{2}+m_{i}^{2}\right) / x_{i}-M^{2}} .
\end{aligned}
$$

These are non-covariant quantities. Note that in this approach energy is not conserved across a vertex, while momentum is conserved. Here, $q_{0}$ is the energy component of the exchanged gauge boson and $E_{i}$ denote the energies of the individual quantum fluctuations over the hadronic background and $E$ is the average energy. The individual momentum fractions are $x_{i}$ with $\sum_{i} x_{i}=1 . m_{i}$ denote the partonic masses and $M$ is the nucleon mass. The parton model is applicable if

$$
R_{\tau}=\frac{\tau_{\text {live }}}{\tau_{\text {int }}} \gg 1
$$

This ratio is a covariant quantity. Yet $p x$ has to be a large momentum. If (4.15) is not fulfilled the exchanged gauge boson is unable to resolve an individual parton.

In the massless case $m_{i}, M=0$ and assuming that all partons having the same transverse momentum one obtains

$$
R_{\tau}=\frac{Q^{2}(1-x)}{2 k_{\perp}^{2} x \sum_{i} \frac{1}{x_{i}}} \approx \frac{Q^{2}(1-x)^{2}}{2 k_{\perp}^{2}}
$$

The last expression in (4.16) is obtained assuming two 'essential' parton with $x_{1}=x, x_{2}=1-x$. Eq. (4.16) is valid for $Q^{2} \gg k_{\perp}^{2}$ and if $x$ neither becomes large or very small, sufficiently away from the elastic and the high energy region. These arguments are qualitative of course. However, close to the excluded regions multi-parton and higher twist effects are expected to contribute essentially.

The momentum fraction $z$ of the nucleon momentum $p$ carried by the single parton depends on the mass of the parton before $\left(m_{I}\right)$ and and after $\left(m_{F}\right)$ the interaction with the intermediate gauge boson as well as the nucleon mass $M$ [148]

$$
z=\frac{Q^{2}+m_{F}^{2}-m_{I}^{2}+\sqrt{\left(Q^{2}+m_{F}^{2}-m_{I}^{2}\right)^{2}+4 m_{I}^{2} Q^{2}}}{2\left(\nu+\sqrt{\nu^{2}+M^{2} Q^{2}}\right)} .
$$


Important special cases are $M=0, m_{I}=0$ and $M=0, m_{I}=m_{F}$,

$$
\begin{aligned}
z\left(M=0, m_{I}=0\right) & =x\left[1+\frac{m_{F}^{2}}{Q^{2}}\right] \\
z\left(M=0, m_{I}=m_{F}\right) & =\frac{x}{2}\left[1+\sqrt{1+\frac{2 m_{F}^{2}}{Q^{2}}}\right] .
\end{aligned}
$$

Since $z \leq 1$ in both cases $x$ is constrained to values smaller than one. For $m_{I}=m_{F}=0, M \neq 0, z$ coincides with the Nachtmann variable $\xi$, (11.1). In the fully massless case the momentum fraction is given by the Bjorken variable

$$
z=x
$$

In many calculations the so-called collinear parton model is used. Here the partons carry the momentum

$$
p^{\prime}=z p, \quad z \in[0,1] .
$$

This Ansatz is sufficient in cases where mass effects or transverse degrees of freedom can be safely neglected.

The covariant parton model generalizes (4.21) accounting for transverse momentum effects and the finite nucleon mass [145, 149, 150]. In Sudakov variables the parton momentum $k$ in terms of the momenta $p$ and $q$, with $p^{2}=M^{2}, p \cdot k_{\perp}=q . k_{\perp}=0, k_{\perp} \cdot k_{\perp}=-k_{\perp}^{2}$ is given by

$$
k=x p+\frac{k^{2}+k_{\perp}^{2}-x^{2} M^{2}}{\frac{Q^{2}}{2}\left[1+\sqrt{1+\frac{4 x^{2} M^{2}}{Q^{2}}}\right]+2 x^{2} M^{2}}\left[\frac{1}{2}\left(1+\sqrt{1+\frac{4 x^{2} M^{2}}{Q^{2}}}\right) q+x p\right]+k_{\perp} .
$$

Here $k^{2}$ denotes the off-shellness of the parton. This representation is used to derive the correct integral relations among polarized structure functions which are sensitive to transverse degrees of freedom, like $g_{2}\left(x, Q^{2}\right)$, cf. Sections 15,16.

\section{$5 \quad$ Renormalization and Factorization and Deep-Inelastic Structure Functions}

The quark masses and the strong coupling are scale dependent quantities due to renormalization, while observables like the structure functions are independent of these arbitrary scales. The scale dependence can be expressed by the operator $\mathcal{D}\left(\mu^{2}\right)$ [47, 48]

$$
\mathcal{D}\left(\mu^{2}\right):=\mu^{2} \frac{\partial}{\partial \mu^{2}}+\beta\left(a_{s}\left(\mu^{2}\right)\right) \frac{\partial}{\partial a_{s}\left(\mu^{2}\right)}-\gamma_{m}\left(a_{s}\left(\mu^{2}\right)\right) m\left(\mu^{2}\right) \frac{\partial}{\partial m\left(\mu^{2}\right)}
$$

with

$$
\begin{aligned}
\beta\left(a_{s}\left(\mu^{2}\right)\right) & =\mu^{2} \frac{\partial a_{s}\left(\mu^{2}\right)}{\partial \mu^{2}} \\
\gamma_{m}\left(a_{s}\left(\mu^{2}\right)\right) & =-\frac{\mu^{2}}{m\left(\mu^{2}\right)} \frac{\partial m\left(\mu^{2}\right)}{\partial \mu^{2}}
\end{aligned}
$$


such that

$$
\left[\mathcal{D}\left(\mu^{2}\right)+\gamma_{J_{1}}+\gamma_{J_{2}}-n_{\psi} \gamma_{\psi}-n_{A} \gamma_{A}\right] F_{i}\left(x, Q^{2}\right)=0
$$

Referring to (3.17) one obtains the following two renormalization group equations for the operator matrix elements and the Wilson coefficients

$$
\begin{gathered}
\sum_{j}\left[\mathcal{D}\left(\mu^{2}\right) \delta_{i j}+\gamma_{i j}^{\mathrm{S}, \mathrm{NS}}-n_{\psi} \gamma_{\psi}-n_{A} \gamma_{A}\right] A_{j}\left(N, \mu^{2}\right)=0 \\
\sum_{j}\left[\mathcal{D}\left(\mu^{2}\right) \delta_{i j}+\gamma_{J_{1}}+\gamma_{J_{2}}-\gamma_{i j}^{\mathrm{S}, \mathrm{NS}}\right] C_{i}\left(N, \frac{Q^{2}}{\mu^{2}}\right)=0 .
\end{gathered}
$$

Here $\gamma_{\psi}, \gamma_{A}$ and $\gamma_{J_{1,2}}$ denote the anomalous dimension of external quarks, gluons, and the currents, which can be non-zero if the currents are not conserved, cf. e.g. [151]. Here the scale $\mu^{2}=\mu_{F}^{2}$ refers to the factorization scale. Furthermore, one may introduce different scales for the renormalization of the coupling and/or the masses. Often these more general scale choices are used for rough estimates of the remaining uncertainties due to higher order corrections.

In the twist-2 approximation the deep-inelastic structure functions obey the following generic representation :

$$
F_{i}\left(x, Q^{2}\right)=\sum_{l} C_{i, l}\left(a_{s}, \frac{Q^{2}}{\mu_{F}^{2}}, m_{c}, m_{b}, x\right) \otimes f^{l}\left(a_{s}, z, \frac{\mu_{F}^{2}}{P^{2}}\right)
$$

with $a_{s}=a_{s}\left(\mu_{R}^{2}\right), m_{c}=m_{c}\left(\mu_{R}^{2}\right), m_{b}=m_{b}\left(\mu_{R}^{2}\right)$, and $\mu_{F, R}$ the factorization and renormalization scales and the sum runs over the corresponding partonic combinations. Furthermore, one has to specify the scheme in which $a_{s}$, resp. $m_{c, b}$ are defined. Performing the perturbative calculations to a given order one may match the $\mu^{2}$-dependence and formulate so-called scheme independent evolution equations for observables, cf. [152 155].

Let us now consider some aspects of the renormalization of the parton distribution functions and the Wilson coefficients. In the calculation we will refer to the $\overline{\mathrm{MS}}$ scheme in $D=4+\varepsilon$ dimensions for the regularization of all singularities, expanding in the dimensional parameter $\varepsilon$. In the calculation of operator matrix elements and Wilson coefficients a universal factor

$$
S_{\varepsilon}=\exp \left[\frac{\varepsilon}{2}\left(\gamma_{E}-\ln (4 \pi)\right)\right]
$$

occurs in each loop order, which is set to one, unlike in the MS-scheme. Here $\gamma_{E}$ denotes the EulerMascheroni constant. To account for mass effects usually different schemes will be used in intermediary steps. The renormalized parton densities $f^{l}$ correspond to strictly massless partons and the coupling constant is that of the $\overline{\mathrm{MS}}$ scheme. So-called heavy quark parton densities will be introduced at a later stage. All heavy quark effects are contained in the Wilson coefficients $C_{i, l}$. Let us now define the massless flavor part of an (inclusive) structure function $F_{i}\left(x, Q^{2}\right), F_{i}^{\text {massless }}\left(x, Q^{2}\right)$. It is given by (5.7) in case the Wilson coefficients do not contain any direct or indirect heavy quark mass effects. Note that this definition is different from one sometimes used in experiment, where one requests all final state fermions being massless. Correspondingly, the massive part of $F_{i}$ is given by

$$
F_{i}^{\text {massive }}\left(x, Q^{2}\right)=F_{i}\left(x, Q^{2}\right)-F_{i}^{\text {massless }}\left(x, Q^{2}\right) \text {. }
$$

The local twist-2 operators (3.11 3.13), resp. the parton distribution functions, are renormalized by

$$
O_{q}^{\mathrm{NS}}=Z^{\mathrm{NS}}\left(\mu^{2}\right) \hat{O}_{q}^{\mathrm{NS}}, \quad O_{i}^{\mathrm{S}}=Z_{i j}^{\mathrm{S}}\left(\mu^{2}\right) \hat{O}_{j}^{\mathrm{S}}
$$


in the non-singlet and singlet case respectively. The $Z$-factors account for the ultraviolet singularities

$$
Z_{i j}^{\alpha}\left(a_{s}, \varepsilon\right)=\delta_{i j}+\sum_{k=0}^{\infty} a_{s}^{l} \sum_{n=1}^{l} \frac{z_{\alpha}^{l, n}}{\varepsilon^{n}}
$$

and the anomalous dimensions given by

$$
\gamma_{q q}^{\mathrm{NS}}=Z^{-1, \mathrm{NS}}\left(\mu^{2}\right) \mu^{2} \frac{\partial}{\partial \mu^{2}} Z^{\mathrm{NS}}\left(\mu^{2}\right), \quad \gamma_{i j}^{\mathrm{S}}=Z_{i l}^{-1, \mathrm{~S}}\left(\mu^{2}\right) \mu^{2} \frac{\partial}{\partial \mu^{2}} Z_{l j}^{\mathrm{S}}\left(\mu^{2}\right)
$$

The unrenormalized coupling $\hat{a}_{s}=g_{s}^{2} /\left(16 \pi^{2}\right)$ is related to the renormalized one by

$$
\hat{a}_{s}=Z_{g}^{2}\left(a_{s}, \varepsilon\right) a_{s}
$$

The heavy quark masses in the Wilson coefficients are renormalized either in the on-shell scheme or the $\overline{\mathrm{MS}}$ scheme,

$$
\hat{m}=Z_{m} m=m\left[1+\sum_{k=1}^{\infty} \hat{a}_{s}^{k}\left(\frac{m^{2}}{\mu^{2}}\right)^{k \varepsilon / 2} \delta m_{k}(\varepsilon)\right] .
$$

The functions $\delta m_{1,2}(\varepsilon)$ have been calculated in [156].

At large enough scales $Q^{2} \gg m_{q}^{2}$ the heavy flavor Wilson coefficients factorize into massive operator matrix elements and the massless Wilson coefficients [71]. In case of the structure function $F_{2}\left(x, Q^{2}\right)$ this is the case for $Q^{2} \gtrsim 10 m_{Q}^{2}$, with $m_{Q}$ the heavy quark mass. In this kinematic regime the renormalization of the heavy flavor Wilson coefficients can be traced back to the massless flavor ones and that of the scheme-independent massive operator matrix elements. As has been lined out in [67] charge renormalization is somewhat more complicated and it is useful to first perform it in a MOM-scheme using the background field method [157] before one transforms to the $\overline{\mathrm{MS}}$-scheme. The massive OMEs contain collinear singularities due to massless sub-graphs, which have to be factorized by the matrices $\Gamma_{i j}^{-1}\left(\mu^{2}\right)$. Unlike the massless case, where

$$
\Gamma_{i j}^{-1}\left(\mu^{2}\right)=Z_{i j}\left(\mu^{2}\right)
$$

this is not the case for the massive OMEs. The renormalization of the massless Wilson coefficients is performed by $\Gamma_{i j}^{-1}\left(\mu^{2}\right)$ taken in the massless case, cf. [61] to 3-loop order. It is evident that the fragile framework of renormalization scarcely allows for any manipulations on 'phenomenological' grounds ad hoc. In any case, in this way a new scheme is chosen.

\section{The strong coupling constant}

The strong coupling $a_{s}\left(\mu^{2}\right)=g_{s}^{2} /\left(16 \pi^{2}\right)=\alpha_{s}\left(\mu^{2}\right) / 4 \pi$ is a central parameter in QCD. It is not an observable itself but the various hard scattering processes are parameterized by it in perturbation theory. Different renormalization schemes may be chosen, cf. [158]. Most commonly $\alpha_{s}\left(\mu^{2}\right)$ is expressed in the $\overline{\mathrm{MS}}$-scheme [159]. All quark flavors are treated as effectively massless.

The running coupling is obtained as the solution of the equation

$$
\frac{d a_{s}\left(\mu^{2}\right)}{d \ln \mu^{2}}=-\beta_{0} a_{s}^{2}-\beta_{1} a_{s}^{3}-\beta_{2} a_{s}^{4}-\beta_{3} a_{s}^{5}+\ldots
$$


So far the contributions to the $\beta$-function have been calculated up to 4 -loop order in the $\overline{\mathrm{MS}}$-scheme, where the LO [35] 38], NLO [160, 161], NNLO [162,163], and $\mathrm{N}^{3} \mathrm{LO}$ [53, 54] terms are given by 4 :

$$
\begin{aligned}
& \beta_{0}=11-\frac{2}{3} N_{f}, \\
& \beta_{1}=102-\frac{38}{3} N_{f}, \\
& \beta_{2}=\frac{2857}{2}-\frac{5033}{18} N_{f}+\frac{325}{54} N_{f}^{2}, \\
& \beta_{3}=\left(\frac{149753}{6}+3564 \zeta_{3}\right)-\left(\frac{1078361}{162}+\frac{6508}{27} \zeta_{3}\right) N_{f}+\left(\frac{50065}{162}+\frac{6472}{81} \zeta_{3}\right) N_{f}^{2}+\frac{1093}{729} N_{f}^{3} .
\end{aligned}
$$

Here we refer to the color coefficients in $S U(3)_{c}$ and $N_{f}$ denotes the number of active flavors. The solution of (6.1) reads [165

$$
\frac{1}{a_{s}\left(Q^{2}\right)}=\frac{1}{a_{s}\left(Q_{0}^{2}\right)}+\beta_{0} \ln \left(\frac{Q^{2}}{Q_{0}^{2}}\right)+\Phi^{(n)}\left(a_{s}\left(Q^{2}\right) ; \beta_{i}\right)-\Phi^{(n)}\left(a_{s}\left(Q_{0}^{2}\right) ; \beta_{i}\right) .
$$

The superscript $n$ denotes the term at which the expansion of the $\beta$-function in (6.1) was truncated. In NNLO one obtains

$$
\Phi^{(2)}\left(x ; \beta_{i}\right)=-\frac{\beta_{1}}{2 \beta_{0}} \ln \left|\frac{x^{2}}{\beta_{0}+\beta_{1} x+\beta_{2} x^{2}}\right|+\frac{\beta_{1}^{2}-2 \beta_{0} \beta_{2}}{\beta_{0} \sqrt{4 \beta_{2} \beta_{0}-\beta_{1}^{2}}} \arctan \left(\frac{\beta_{1}+2 \beta_{2} x}{\sqrt{4 \beta_{0} \beta_{2}-\beta_{1}^{2}}}\right) .
$$

Note that

$$
\begin{array}{ll}
N_{f} \leq 5: & 4 \beta_{0} \beta_{2}-\beta_{1}^{2}>0 \\
N_{f}=6: & 4 \beta_{0} \beta_{2}-\beta_{1}^{2}<0 .
\end{array}
$$

This explains the smaller change in $a_{s}\left(\mu^{2}\right)$ from NLO to NNLO at scales below the top threshold, if compared to the logarithmic corrections characteristic for NLO.

Often (6.1) is solved expanding the approximate polynomial in $a_{s}$ after the separation of variables. Let us define $L=\ln \left(\mu^{2} / \Lambda^{2}\right)$. Then, according to the convention in [159], one obtains [166] :

$$
\begin{aligned}
a_{s}\left(\mu^{2}\right)= & \frac{1}{\beta_{0} L}-\frac{\beta_{1}}{\beta_{0}^{3} L^{2}} \ln (L)+\frac{1}{\beta_{0}^{3} L^{3}}\left[\frac{\beta_{1}^{2}}{\beta_{0}^{2}}\left(\ln ^{2}(L)-\ln (L)-1\right)+\frac{\beta_{2}}{\beta_{0}}\right] \\
& +\frac{1}{\beta_{0}^{4} L^{4}}\left[\frac{\beta_{1}^{3}}{\beta_{0}^{3}}\left(-\ln ^{3}(L)+\frac{5}{2} \ln ^{2}(L)+2 \ln (L)-\frac{1}{2}\right)-3 \frac{\beta_{1} \beta_{2}}{\beta_{0}^{2}} \ln (L)+\frac{\beta_{3}}{2 \beta_{0}}\right] .
\end{aligned}
$$

Here $\Lambda \equiv \Lambda\left(N_{f}\right)$ denotes the QCD scale. In applications the scale $\mu^{2}$ varies over a wider range and may pass flavor thresholds. As a convention, one identifies the scale $\mu$ at which a new quark flavor becomes active by $m_{q}, q=c, b, t$. This definition implies matching conditions in $a_{s}\left(\mu^{2}\right)$. At LO and NLO they are given by $a_{s}\left(N_{f}-1\right)=a_{s}\left(N_{f}\right)$, while in higher orders more specific conditions apply [166 168]. For 4-loop running the 3-loop matching conditions are

$$
\frac{a_{s}\left(N_{f}-1\right)}{a_{s}\left(N_{f}\right)}=1+C_{2} a_{s}^{2}\left(N_{f}\right)+C_{3}\left(N_{f}\right) a_{s}^{3}\left(N_{f}\right)
$$

with $C_{2}=-14 / 3, C_{3}=-340.7289736+16.79813197\left(N_{f}-1\right)$ for $\mu=m_{q}$. In Section 12 we will summarize the current status of the determination of $\alpha_{s}\left(M_{Z}^{2}\right)$ from deep-inelastic data.

\footnotetext{
${ }^{4}$ For Quantum Electrodynamics also the coefficient $\beta_{4}=195067 / 486+(800 / 3) \zeta_{3}+(416 / 3) \zeta_{4}-(6800 / 3) \zeta_{5}$ has been calculated in [164.
} 


\section{Anomalous Dimensions and Splitting Functions}

The anomalous dimensions of local twist-2 operators $\gamma_{i j}\left(a_{s}, N\right)$ have the representation

$$
\gamma_{i j}^{l}\left(a_{s}, N\right)=\sum_{k=1}^{\infty} a_{s}\left(\mu^{2}\right) \gamma_{i j}^{l,(k-1)}(N)
$$

where $N$ denotes the Mellin variable. They are related to the splitting functions in $z$-space by

$$
\gamma_{i j}^{l,(k-1)}(N)=-\int_{0}^{1} d z z^{N-1} P_{i j}^{l,(k-1)}(z)
$$

uniquely [169], cf. also [170]. $z$ denotes the collinear momentum fraction of the parton compared to its emitting particle.

Here $l$ labels the three flavor non-singlet $\left(\mathrm{NS}^{ \pm}, \mathrm{NS}^{\mathrm{v}}\right)$ and singlet $(\mathrm{S})$ cases, with partonic transitions $j \rightarrow i$. The three flavor non-singlet splitting functions are given by, cf. [59],

$$
\begin{aligned}
& \gamma_{q q}^{\mathrm{NS}, \pm}(N)=\gamma_{q q}^{\mathrm{v}}(N) \pm \gamma_{q \bar{q}}^{\mathrm{v}}(N) \\
& \gamma_{q q}^{\mathrm{NS}, \mathrm{v}}(N)=\gamma_{q q}^{\mathrm{v}}(N)-\gamma_{q \bar{q}}^{\mathrm{v}}(N)+N_{f}\left(\gamma_{q q}^{\mathrm{s}}(N)-\gamma_{q \bar{q}}^{\mathrm{s}}(N)\right)
\end{aligned}
$$

with

$$
\begin{aligned}
& \gamma_{q_{i} q_{j}}(N)=\gamma_{\bar{q}_{j} \bar{q}_{i}}(N)=\delta_{i j} \gamma_{q q}^{\mathrm{v}}(N)+\gamma_{q q}^{\mathrm{s}}(N) \\
& \gamma_{q_{i} \bar{q}_{j}}(N)=\gamma_{\bar{q}_{i} q_{j}}(N)=\delta_{i j} \gamma_{q \bar{q}}^{\mathrm{v}}(N)+\gamma_{q \bar{q}}^{\mathrm{s}}(N) .
\end{aligned}
$$

$\gamma_{q \bar{q}}^{\mathrm{v}}(N)$ contributes for the first time at NLO and the non-zero difference $\gamma_{q q}^{\mathrm{s}}(N)-\gamma_{q \bar{q}}^{s}(N)$ emerges with NNLO. Up to exceptions the anomalous dimensions are different in the unpolarized and polarized cases. Note that there are sometimes different conventions being used in the literature parameterizing the anomalous dimensions, splitting functions and Wilson coefficients, due to a different normalization of the strong coupling constant or using the operator $\mu \partial / \partial \mu$ instead of $\mu^{2} \partial / \partial \mu^{2}$ in the renormalization group equation. Fermion-number conservation implies

$$
\begin{aligned}
& \gamma_{q q}^{\mathrm{NS},-}(1)=0 \\
& \gamma_{q q}^{\mathrm{NS}, \mathrm{v}}(1)=0
\end{aligned}
$$

\subsection{Leading Order}

The leading order QCD anomalous dimensions in the unpolarized case have been calculated in [171-173]. They are given by

$$
\begin{aligned}
& \gamma_{q q}^{(0)}(N)=C_{F}\left[4 S_{1}(N)-\frac{3 N^{2}+3 N+2}{N(N+1)}\right]=\gamma_{q q}^{(0), \mathrm{NS}}(N) \\
& \gamma_{q g}^{(0)}(N)=-4 T_{F} N_{f} \frac{2+N+N^{2}}{N(N+1)(N+2)} \\
& \gamma_{g q}^{(0)}(N)=-2 C_{F} \frac{2+N+N^{2}}{(N-1) N(N+1)} \\
& \gamma_{g g}^{(0)}(N)=C_{A}\left[-\frac{\left.24+2 N+13 N^{2}+22 N^{3}+11 N^{4}\right)}{3(N-1) N(1+N)(2+N)}+4 S_{1}(N)\right]+\frac{8}{3} T_{F} N_{f},
\end{aligned}
$$


resp. in $z$-space

$$
\begin{aligned}
& P_{q q}^{(0)}(z)=2 C_{F}\left(\frac{1+z^{2}}{1-z}\right)_{+} \\
& P_{q g}^{(0)}(z)=4 T_{f} N_{f}\left[z^{2}+(1-z)^{2}\right] \\
& P_{g q}^{(0)}(z)=2 C_{F} \frac{1+(1-z)^{2}}{z} \\
& P_{g g}^{(0)}(z)=4 C_{A}\left[\frac{1}{(1-z)_{+}}+\frac{1}{z}-2+z-z^{2}\right]+2 \beta_{0} \delta(1-z) .
\end{aligned}
$$

Here, $S_{1}(N)$ denotes the simplest of the nested harmonic sums [174].

$$
S_{b, a_{1}, \ldots, a_{n}}(N)=\sum_{k=1}^{N} \frac{(\operatorname{sign}(b))^{k}}{k^{|b|}} S_{a_{1}, \ldots, a_{n}}(k), \quad S_{\emptyset}=1 .
$$

The structure of the splitting functions $P_{q q}^{(0)}, P_{q g}^{(0)}, P_{g q}^{(0)}$ has been known from QED, [175, see also [176, [177]. Following [50,51] splitting functions for fermion-pseudoscalar and fermion-abelian vector theories

$$
\mathcal{L}=\bar{\psi} \gamma_{5} \psi \Phi, \quad \mathcal{L}=\bar{\psi} \gamma_{\mu} \psi A^{\mu}
$$

were calculated in [178 181]. For Quantum Chromodynamics they were computed in Refs. [182 184] in the space-like resp. in [185] in the time-like case. In [183, 186] also the individual helicity contributions were given, [187]. The calculation of the QCD splitting functions [183] was of importance to extend the naive parton model to the QCD improved parton model. The notion of splitting functions, furthermore, forms a more intuitive picture, if compared to the more formal description obtained from QCD corrections to the amplitudes in the light cone expansion, and contributed a lot to the detailed understanding of the respective processes.

The following relations apply for $z<1$ :

$$
\begin{aligned}
& P_{q q}(z)=P_{g q}(1-z) \\
& P_{q g}(z)=P_{q g}(1-z) \\
& P_{g g}(z)=P_{g g}(1-z) .
\end{aligned}
$$

Furthermore, the integral relations

$$
\begin{aligned}
\int_{0}^{1} d z z\left[P_{q q}(z)+P_{g q}(z)\right] & =0 \\
\int_{0}^{1} d z z\left[2 N_{f} P_{q g}(z)+P_{g g}(z)\right] & =0
\end{aligned}
$$

hold.

In the polarized case the leading order singlet anomalous dimensions were calculated in [188, 189] 5 using the operator approach and in Ref. [183] for the splitting functions :

$$
\begin{aligned}
\Delta \gamma_{q q}^{(0)}(N) & =\gamma_{q q}^{(0), \mathrm{NS}}(N)=\Delta \gamma_{q q}^{(0), \mathrm{NS}}(N) \\
\Delta \gamma_{q g}^{(0)}(z) & =-4 T_{f} N_{f} \frac{N+2}{N(N+1)} \\
\Delta \gamma_{g q}^{(0)}(z) & =-2 C_{F} \frac{N-1}{N(N+1)} \\
\Delta \gamma_{g g}^{(0)}(z) & =4 C_{A}\left[S_{1}(N)-\frac{2}{N(N+1)}\right]-2 \beta_{0} .
\end{aligned}
$$

\footnotetext{
${ }^{5}$ The foregoing paper [190] was not fully correct.
} 


\section{$7.2 \quad$ Next-to-Leading Order}

The splitting functions at NLO were calculated in [191,192]. For space-like virtualities they read :

$$
\begin{aligned}
& \gamma_{q q}^{\mathrm{NS},-,(1)}(N)=C_{A} C_{F}\left[-16 S_{-2,1}-\frac{51 N^{5}+102 N^{4}+655 N^{3}+484 N^{2}+12 N+144}{18 N^{3}(N+1)^{2}}+8 S_{-3}+\frac{268}{9} S_{1}\right. \\
& \left.+\left(16 S_{1}-\frac{8}{N(N+1)}\right) S_{2}-\frac{44}{3} S_{2}+8 S_{3}-\frac{8(-1)^{N}}{(N+1)^{3}}\right]+C_{F}^{2}\left[32 S_{-2,1}+\left(\frac{8(2 N+1)}{N^{2}(N+1)^{2}}\right.\right. \\
& \left.-16 S_{2}\right) S_{1}+\frac{4\left(3 N^{2}+3 N+2\right)}{N(N+1)} S_{2}-\frac{3 N^{6}+9 N^{5}+9 N^{4}-5 N^{3}-24 N^{2}-32 N-24}{2 N^{3}(N+1)^{3}} \\
& \left.-16 S_{-3}+\left(\frac{16}{N(N+1)}-32 S_{1}\right) S_{2}-16 S_{3}(N)+\frac{16(-1)^{N}}{(N+1)^{3}}\right] \\
& +C_{F} N_{f}\left(\frac{3 N^{4}+6 N^{3}+47 N^{2}+20 N-12}{9 N^{2}(N+1)^{2}}-\frac{40}{9} S_{1}+\frac{8}{3} S_{2}\right) \\
& \gamma_{q q}^{\mathrm{PS},(1)}(N)=-4 C_{F} N_{f} \frac{\left(2+5 N+N^{2}\right)\left(4+4 N+7 N^{2}+5 N^{3}\right)}{(-1+N) N^{3}(1+N)^{3}(2+N)^{2}} \\
& \gamma_{q g}^{(1)}(N)=C_{A} N_{f}\left\{\frac{16(-1)^{N}\left(5+7 N+4 N^{2}+N^{3}\right)}{(1+N)^{3}(2+N)^{3}}-\frac{4 P_{1}(N)}{(-1+N) N^{3}(1+N)^{2}(2+N)^{3}}\right. \\
& \left.-\frac{16(3+2 N)}{(1+N)^{2}(2+N)^{2}} S_{1}+\frac{4\left(2+N+N^{2}\right)}{N(1+N)(2+N)}\left(2 S_{-2}+S_{1}^{2}+S_{2}\right)\right\} \\
& +C_{F} N_{f}\left\{\frac{-2\left(4+8 N+25 N^{2}+51 N^{3}+36 N^{4}+15 N^{5}+5 N^{6}\right)}{N^{3}(1+N)^{3}(2+N)}\right. \\
& \left.+\frac{8}{N^{2}} S_{1}+\frac{4\left(2+N+N^{2}\right)}{N(1+N)(2+N)}\left(-S_{1}^{2}+S_{2}\right)\right\} \\
& \gamma_{g q}^{(1)}(N)=C_{F}^{2}\left\{\frac{2\left(-4-12 N-N^{2}+28 N^{3}+43 N^{4}+30 N^{5}+12 N^{6}\right)}{(-1+N) N^{3}(1+N)^{3}}\right. \\
& \left.-\frac{4\left(10+17 N+8 N^{2}+5 N^{3}\right)}{(-1+N) N(1+N)^{2}} S_{1}+\frac{4\left(2+N+N^{2}\right)}{(-1+N) N(1+N)}\left(S_{1}^{2}+S_{2}\right)\right\} \\
& +C_{A} C_{F}\left\{\frac{-8(-1)^{N}\left(2+6 N+5 N^{2}+3 N^{3}\right)}{(-1+N) N^{2}(1+N)^{3}}-\frac{4 P_{2}(N)}{9(-1+N)^{2} N^{3}(1+N)^{2}(2+N)^{2}}\right. \\
& +\frac{4\left(-12-22 N+41 N^{2}+17 N^{4}\right)}{3(-1+N)^{2} N^{2}(1+N)} S_{1}+\frac{4\left(2+N+N^{2}\right)}{(-1+N) N(1+N)}\left(S_{-2}-S_{1}^{2}+S_{2}\right) \\
& +C_{F} N_{f}\left\{\frac{8\left(16+27 N+13 N^{2}+8 N^{3}\right)}{9(-1+N) N(1+N)^{2}}-\frac{8\left(2+N+N^{2}\right)}{3(-1+N) N(1+N)} S_{1}\right\} \\
& \gamma_{g g}^{(1)}(N)=C_{A}^{2}\left\{\frac{-16(-1)^{N}\left(8+36 N+61 N^{2}+61 N^{3}+36 N^{4}+12 N^{5}+2 N^{6}\right)}{(-1+N) N^{2}(1+N)^{3}(2+N)^{3}}\right. \\
& -\frac{2 P_{3}(N)}{9(-1+N)^{2} N^{3}(1+N)^{2}(2+N)^{3}}-8 S_{-3}+\left[\frac{32\left(1+N+N^{2}\right)}{(-1+N) N(1+N)(2+N)}-16 S_{1}\right] S_{-2}
\end{aligned}
$$




$$
\begin{aligned}
& +\left[\frac{4 P_{4}(N)}{9(-1+N)^{2} N^{2}(1+N)^{2}(2+N)^{2}}-16 S_{2}\right] S_{1}+\frac{32\left(1+N+N^{2}\right)}{(-1+N) N(1+N)(2+N)} S_{2} \\
& \left.-8 S_{3}+16 S_{-2,1}\right\} \\
& +C_{A} N_{f}\left[\frac{8\left(6+28 N+41 N^{2}+29 N^{3}+22 N^{4}+9 N^{5}+3 N^{6}\right)}{9(-1+N) N^{2}(1+N)^{2}(2+N)}-\frac{40}{9} S_{1}\right] \\
& +2 C_{F} N_{f} \frac{P_{5}(N)}{(-1+N) N^{3}(1+N)^{3}(2+N)}
\end{aligned}
$$

with

$$
\begin{aligned}
P_{1}(N)= & 16+48 N+56 N^{2}+52 N^{3}+25 N^{4}+23 N^{5}+14 N^{6}+5 N^{7}+N^{8} \\
P_{2}(N)= & 144+432 N-152 N^{2}-900 N^{3}-275 N^{4}+592 N^{5}+834 N^{6}+512 N^{7}+109 N^{8} \\
P_{3}(N)= & 576+1488 N+1088 N^{2}-536 N^{3}-848 N^{4}+711 N^{5}+2075 N^{6}+1949 N^{7}+937 N^{8} \\
& +288 N^{9}+48 N^{10} \\
P_{4}(N)= & -144-144 N+772 N^{2}+844 N^{3}-109 N^{4}-392 N^{5}+134 N^{6}+268 N^{7}+67 N^{8} \\
P_{5}(N)= & -8-8 N-10 N^{2}-22 N^{3}-3 N^{4}+6 N^{5}+8 N^{6}+4 N^{7}+N^{8} .
\end{aligned}
$$

In the polarized case the anomalous dimensions were obtained in [69, 70] :

$$
\begin{aligned}
& \Delta \gamma_{q q}^{\mathrm{NS},-,(1)}(N)=\gamma_{q q}^{\mathrm{NS},-,(1)}(N) \\
& \Delta \gamma_{q q}^{\mathrm{PS},(1)}(N)=16 C_{F} T_{F} N_{f} \frac{(N+2)\left(N^{3}+2 N+1\right)}{N^{3}(N+1)^{3}} \\
& \Delta \gamma_{q g}^{(1)}(N)=16 C_{A} T_{F} N_{f}\left\{\frac{N-1}{N(N+1)}\left[S_{1}^{2}+S_{2}+2 S_{-2}\right]-\frac{4}{N(N+1)^{2}} S_{1}\right. \\
& \left.-\frac{N^{5}+N^{4}-4 N^{3}+3 N^{2}-7 N-2}{N^{3}(N+1)^{3}}\right\}+8 C_{F} T_{F} N_{f}\left\{4 \frac{(N-1)}{N^{2}(N+1)} S_{1}\right. \\
& \left.-\frac{(N-1)\left(5 N^{4}+10 N^{3}-N+2\right)}{N^{3}(N+1)^{3}}+2 \frac{(N-1)}{N(N+1)}\left[S_{2}-S_{1}^{2}\right]\right\} \\
& \Delta \gamma_{g q}^{(1)}(N)=8 C_{A} C_{F}\left\{\frac{N+2}{N(N+1)}\left[-S_{1}^{2}+S_{2}+2 S_{-2}\right]+\frac{11 N^{2}+22 N+12}{3 N^{2}(N+1)} S_{1}\right. \\
& \left.-\frac{76 N^{5}+271 N^{4}+254 N^{3}+41 N^{2}+72 N+36}{9 N^{3}(N+1)^{3}}\right\}+4 C_{F}^{2}(N+2)\left\{2 \frac{1}{N(N+1)}\right. \\
& \left.\times\left[S_{1}^{2}+S_{2}\right]-2 \frac{3 N+1}{N(N+1)^{2}} S_{1}+\frac{(3 N+1)\left(3 N^{3}+3 N^{2}-N-2\right)}{N^{3}(N+1)^{3}}\right\} \\
& +32 C_{F} N_{f} T_{F}(N+2)\left[\frac{5 N+2}{9 N(N+1)^{2}}-\frac{1}{3 N(N+1)} S_{1}\right] \\
& \Delta \gamma_{g g}^{(1)}=4 C_{A}^{2}\left\{\frac{2\left(67 N^{4}+134 N^{3}+67 N^{2}+144 N+72\right)}{9 N^{2}(N+1)^{2}} S_{1}-8 S_{1}\left[S_{2}+S_{-2}\right]\right. \\
& -\frac{48 N^{6}+144 N^{5}+469 N^{4}+698 N^{3}+7 N^{2}+258 N+144}{9 N^{3}(N+1)^{3}}+16 \frac{S_{2}+S_{-2}}{N(N+1)}
\end{aligned}
$$




$$
\begin{aligned}
& \left.+8 S_{-2,1}-4 S_{3}-4 S_{-3}\right\} \\
& +32 C_{A} T_{F} N_{f}\left[\frac{3 N^{4}+6 N^{3}+16 N^{2}+13 N-3}{9 N^{2}(N+1)^{2}}-\frac{5}{9} S_{1}\right] \\
& +8 C_{F} T_{F} N_{f} \frac{N^{6}+3 N^{5}+5 N^{4}+N^{3}-8 N^{2}+2 N+4}{N^{3}(N+1)^{3}}
\end{aligned}
$$

In the above expressions one may identify the leading behaviour at small $x$ given by poles at $N=1$ resp. $N=0$, cf. Section 13. The leading large $x$ behaviour manifests itself by powers of $S_{1}(N) \propto \ln (N)$ in the limit $N \rightarrow \infty$, cf. Section 14 .

\subsection{3-Loop Order}

The unpolarized 3-loop anomalous dimensions have first been calculated for a series of fixed moments in [55, 58] and then in complete form in [59,60]. Independent checks for the moments were obtained as a by-product of the calculation of the massive operator matrix elements [67] for all color factors $\propto T_{F}$ and for the leading $N_{f}$ dependence [193 195]. In Mellin-space the anomalous dimensions may be represented by basic harmonic sums up to weight $\mathrm{w}=5$ [196]. Analytic continuations for these functions to complex values of $N$ were given in [197]. In $z$-space they are given by harmonic polylogarithms [198]. The corresponding expressions are very lengthly and will not be presented here. At a given value of $\alpha_{s}$ their size can be compared to the corresponding values at LO and NLO, cf. Figure 9. As present QCD-anlyses show, the seemingly small difference between the curves at NLO and NNLO account for a difference of $\Delta \alpha_{s}\left(M_{Z}^{2}\right) \approx 0.005$, which is essential given the experimental accuracy of $\sim 1 \%$ being reached at present, see Section 12 .
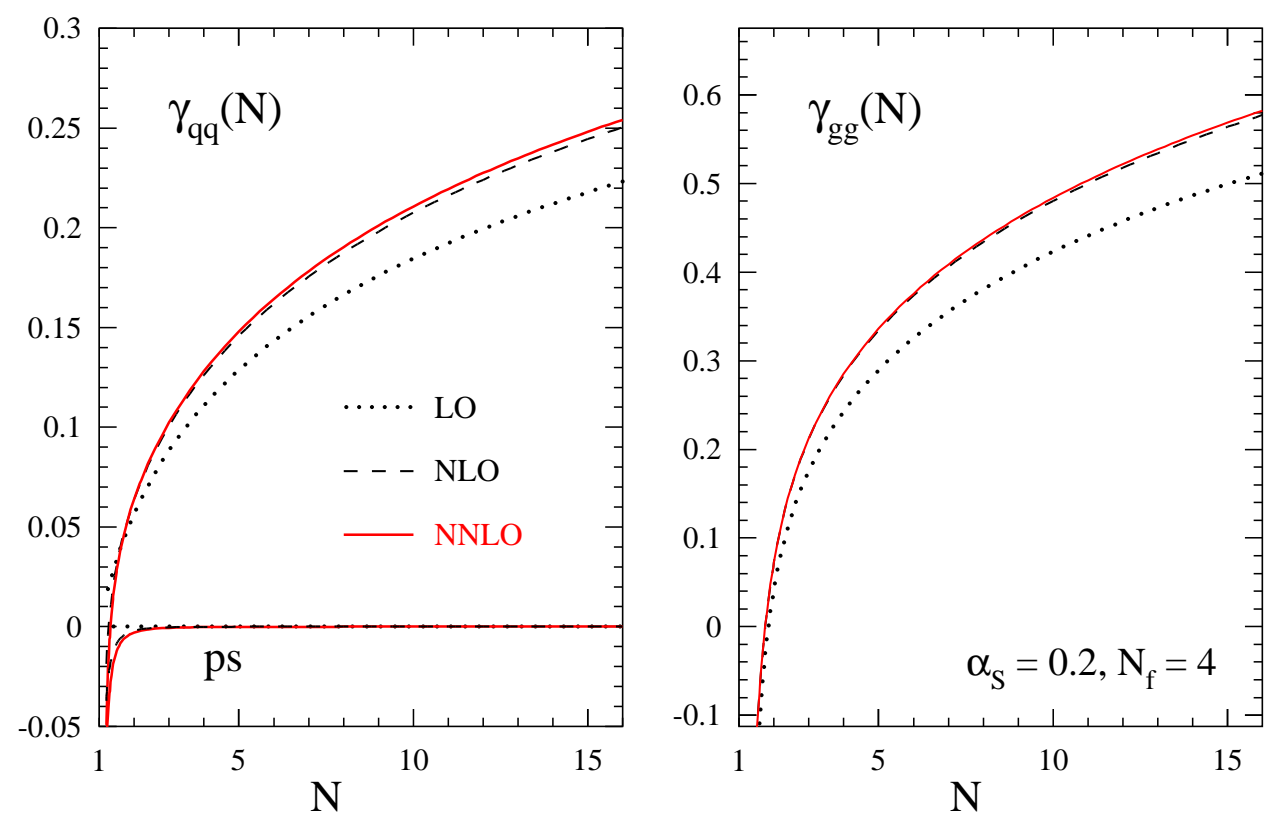

Figure 9: The perturbative expansion of the diagonal anomalous dimensions $\gamma_{q q}(N)$ and $\gamma_{g g}(N)$ for four flavors at $\alpha_{s}=0.2$. The pure-singlet (ps) contribution to $\gamma_{q q}(N)$ is shown separately. From [60], (C)(2004) by Elsevier Science.

First numeric results on the quarkonic contributions to the 3-loop polarized anomalous dimensions were given in [73]. 


\subsection{4-Loop Order}

At the 4-loop level at present the 2 nd and 3rd moment of the non-singlet anomalous dimension have been calculated [62 64]. Recently the general color structure of $\gamma^{(3), N S}(N=2)$ has been given in [64].

$$
\begin{aligned}
\gamma_{N_{f}=3}^{(3), \mathrm{NS}}(N=2)= & \frac{1680283336}{177147}-\frac{23873952}{6561} \zeta_{3}+\frac{5120}{3} \zeta_{4}-\frac{56960}{243} \zeta_{5},[62], \\
\gamma_{S U(3)_{c}}^{(3), \mathrm{NS}}(N=2)= & {\left[\frac{3100369144}{177147}+\frac{26060864}{6561} \zeta_{3}-\frac{7040}{27} \zeta_{4}-\frac{1249280}{243} \zeta_{5}\right] } \\
& +\left[-\frac{167219672}{59049}-\frac{6322976}{2187} \zeta_{3}+\frac{64640}{81} \zeta_{4}+\frac{14720}{9} \zeta_{5}\right] N_{f} \\
& +\left[\frac{1084904}{19683}+\frac{2560}{27} \zeta_{3}-\frac{1280}{27} \zeta_{4}\right] N_{f}^{2}+\left[-\frac{4096}{6561}+\frac{512}{243} \zeta_{3}\right] N_{f}^{3}, \text { 64]. }
\end{aligned}
$$

In numerical studies the 4-loop [62,63] moments were compared to the result of a Padé approximation [199.

$$
\gamma_{\mathrm{NS}}^{(3), \text { Padé }}(N)=\frac{\gamma_{\mathrm{NS}}^{(2)}(N)^{2}}{\gamma_{\mathrm{NS}}^{(1)}(N)}
$$

for $N_{f}=3$, as genuine number of massless flavors. For the second moment the Padé approximation and the exact result deviate by $20.9 \%$ and for the third moment by $14.9 \%$ only. In Ref. [199] an uncertainty of $\pm 100 \%$ has been assumed for (7.44).

\subsection{Anomalous dimensions in the large $N_{f}$ limit}

The leading and sub-leading coefficients in $1 / N_{f}$ can be calculated for the anomalous dimensions and the $\beta$-function using the method of Refs. [200] to all orders in the coupling constant in the $\overline{\mathrm{MS}}$-scheme. The result of these computations give very useful predictions for the complete diagrammatic calculation, which is more difficult. Results were obtained for the fermion mass anomalous dimensions [201 203], the QCD $\beta$-function [204], the unpolarized anomalous dimensions of composite twist-2 operators [193, 205], the flavor non-singlet Wilson coefficient of $F_{L}\left(x, Q^{2}\right)$ [206], the polarized anomalous dimensions [207], and the anomalous dimensions for transversity [208]. Here, the electron and quark mass anomalous dimension [201,202] have been calculated to $O\left(1 / N_{f}^{2}\right)$. As one example we show the prediction for the 4-loop flavor non-singlet anomalous dimension

$$
\begin{aligned}
\gamma^{(3), \mathrm{NS}}(N)= & C_{F}\left(T_{R} N_{f}\right)^{3}\left\{\frac{2}{27} S_{4}(N)-\frac{10}{81} S_{3}(N)-\frac{2}{81} S_{2}(N)-\frac{2}{81} S_{1}(N)+\frac{131}{1296}\right. \\
& \left.+\left[\frac{4}{27} S_{1}(N)-\frac{2}{27 N(N+1)}-\frac{1}{9}\right] \zeta_{3}+\frac{(2 N-1)\left(2 N^{5}-5 N^{4}-10 N^{3}+7 N+3\right)}{81 N^{4}(N+1)^{4}}\right\}
\end{aligned}
$$

which has been confirmed by an explicit calculation for $N=2$ [62, 64] and $N=3$ [63]. Another example concerns a combination of gluonic anomalous dimensions. At 3-loop order one obtains

$$
\tilde{\gamma}_{g g}^{(2)}+\tilde{\gamma}_{g q}^{(2)} \frac{\gamma_{q g}^{(0)}}{\tilde{\gamma}_{g g}^{(0)}}=C_{F} T_{R}^{2} N_{f}^{2}\left\{\frac{64}{3} \frac{\left(N^{2}+N+2\right)^{2}}{(N+2)(N+1)^{2}(N-1) N^{2}} S_{1}^{2}-\frac{4}{27} \frac{p_{1}(N)}{(N+2)(N+1)^{4}(N-1) N^{4}}\right.
$$




$$
\begin{aligned}
& \left.-\frac{64}{9} \frac{10 N^{6}+30 N^{5}+109 N^{4}+168 N^{3}+155 N^{2}+76 N+12}{(N+2)(N+1)^{3}(N-1) N^{3}} S_{1}\right\} \\
+ & C_{A} T_{R}^{2} N_{f}^{2}\left\{\frac{8}{27} \frac{8 N^{6}+24 N^{5}-19 N^{4}-78 N^{3}-253 N^{2}-210 N-96}{(N+2)(N+1)^{2}(N-1) N^{2}} S_{1}\right. \\
& \left.-\frac{2}{27} \frac{p_{2}(N)}{(N+2)(N+1)^{3}(N-1) N^{3}}\right\} \\
p_{1}(N)= & 33 N^{10}+165 N^{9}-32 N^{8}-1118 N^{7}-5807 N^{6}-12815 N^{5}-16762 N^{4}-13800 N^{3} \\
p_{2}(N)= & -7112 N^{2}-2112 N-288
\end{aligned}
$$

Here $\tilde{\gamma}_{i j}^{(l)}$ denotes the respective part of highest power in $N_{f}$. The result was confirmed in [60], and more recently also in [195].

\section{Coefficient functions}

The Wilson coefficients in Mellin space obey the following perturbative expansion

$$
C_{i}\left(N, a_{s}\right)=\delta_{i q}+\sum_{k=1}^{\infty} a_{s}\left(\mu^{2}\right) C_{i}^{(k)}(N)
$$

Similar expressions are also obtained for other hard scattering inclusive cross sections depending on a single momentum-fraction scale like for the Drell-Yan and hadronic Higgs-boson production cross section in the heavy top-quark approximation [209,210].

\subsection{First Order}

The first order unpolarized coefficient functions in the massless case in the $\overline{\mathrm{MS}}$-scheme are given by, cf. 152 :

$$
\begin{aligned}
& C_{2, q}^{(1)}(N)=C_{F}\left\{-\frac{9 N^{3}+2 N^{2}-5 N-2}{N^{2}(1+N)}+\frac{3 N^{2}+3 N-2}{N(1+N)} S_{1}+2 S_{1}^{2}-2 S_{2}\right\} \\
& C_{2, g}^{(1)}(N)=N_{f}\left\{\frac{-2\left(-2-N-4 N^{2}+N^{3}\right)}{N^{2}(1+N)(2+N)}-\frac{2\left(2+N+N^{2}\right)}{N(1+N)(2+N)} S_{1}\right\} \\
& C_{L, q}^{(1)}(N)=\frac{4 C_{F}}{1+N} ; \quad C_{L, g}^{(1)}(N)=\frac{8 N_{f}}{(1+N)(2+N)} \\
& C_{3, q}^{(1)}(N)=C_{2, q}^{(1)}(N)-2 C_{F} \frac{2 N+1}{N(N+1)} .
\end{aligned}
$$

Likewise the LO massless polarized Wilson coefficients for the twist-2 structure function $g_{1}\left(x, Q^{2}\right)$ read, [211 214], see also [215] :

$$
\begin{aligned}
\Delta C_{1, q}^{(1)}(N) & =C_{3, q}^{(1)}(N) \\
\Delta C_{1, g}^{(1)}(N) & =2 N_{f} \frac{N-1}{N(N+1)}\left[\frac{1}{N}-1-S_{1}\right] .
\end{aligned}
$$

The first moment of $\Delta C_{1, g}^{(1)}(N)$ vanishes, which is also observed for $\Delta C_{1, g}^{(2)}(N)$, [68]. 


\subsection{Higher Orders}

The massless Wilson coefficients for the unpolarized structure functions at $O\left(a_{s}^{2}\right)$ were calculated in [192,216] for $F_{2}\left(x, Q^{2}\right)$, in [192,216, 217] for $F_{L}\left(x, Q^{2}\right)$, and for $x F_{3}\left(x, Q^{2}\right)$ in [218]. A series of moments was calculated in [219]. The polarized $O\left(a_{s}^{2}\right)$ Wilson coefficients for the structure function $g_{1}\left(x, Q^{2}\right)$ were computed in [68]. All Wilson coefficients can be expressed in terms of harmonic sums up to weight $\mathrm{w}=4$. As an example we show the coefficient functions in case of $F_{L}\left(x, Q^{2}\right)$, with the quarkonic, the pure-singlet and the gluonic contribution :

$$
\begin{aligned}
& C_{L, q}^{(2)}(N)=C_{A} C_{F}\left\{\left(\frac{32}{N+1} S_{1}-\frac{32\left(N^{4}+2 N^{3}-N^{2}-2 N-6\right)}{(N-2) N(N+1)^{2}(N+3)}\right) S_{-2}+\frac{16}{N+1}\left[S_{-3}+S_{3}\right]\right. \\
& +\frac{92}{3(N+1)} S_{1}-\frac{32}{N+1} S_{-2,1}-\frac{16(-1)^{N} P_{6}(N)}{5(N-2)(N-1)^{2} N^{2}(N+1)^{4}(N+2)^{2}(N+3)^{3}} \\
& \left.+\frac{2 P_{7}(N)}{45(N-1)^{2} N^{2}(N+1)^{4}(N+2)^{2}(N+3)^{3}}+\frac{48}{N+1} \zeta_{3}\right\}+C_{F}^{2}\left\{-\frac{4\left(9 N^{2}+13 N+2\right)}{N(N+1)^{2}} S_{1}\right. \\
& +\left(\frac{64\left(N^{4}+2 N^{3}-N^{2}-2 N-6\right)}{(N-2) N(N+1)^{2}(N+3)}-\frac{64}{N+1} S_{1}\right) S_{-2}+\frac{8}{N+1} S_{1}^{2}-\frac{32}{N+1}\left[S_{-3}+S_{3}\right] \\
& -\frac{8}{N+1} S_{2}+\frac{64}{N+1} S_{-2,1}+\frac{32(-1)^{N} P_{6}(N)}{5(N-2)(N-1)^{2} N^{2}(N+1)^{4}(N+2)^{2}(N+3)^{3}} \\
& \left.-\frac{2 P_{8}(N)}{5(N-1)^{2} N^{2}(N+1)^{4}(N+2)^{2}(N+3)^{3}}+\frac{96}{N+1} \zeta_{3}\right\} \\
& +C_{F} N_{F}\left[\frac{-4\left(19 N^{2}+7 N-6\right)}{9 N(N+1)^{2}}-\frac{8}{3(N+1)} S_{1}\right] \\
& C_{L, q}^{(2), \mathrm{PS}}(N)=C_{F} N_{F}\left[\frac{-16\left(N^{5}+2 N^{4}+2 N^{3}-5 N^{2}-12 N-4\right)}{(N-1) N^{2}(N+1)^{3}(N+2)^{2}}-\frac{16\left(N^{2}+N+2\right)}{(N-1) N(N+1)^{2}(N+2)} S_{1}\right] \\
& C_{L, g}^{(2)}(N)=C_{A} C_{F}\left\{\frac{32\left(2 N^{3}-2 N^{2}-N-1\right)}{(N-1) N(N+1)^{2}(N+2)} S_{1}+\frac{16}{(N+1)(N+2)} S_{1}^{2}-\frac{32}{(N+1)(N+2)} S_{-2}\right. \\
& -\frac{16}{(N+1)(N+2)} S_{2}+\frac{32(-1)^{N}\left(N^{3}+4 N^{2}+7 N+5\right)}{(N+1)^{3}(N+2)^{3}} \\
& \left.-\frac{16\left(2 N^{5}+9 N^{4}+5 N^{3}-12 N^{2}-20 N-8\right)}{(N-1) N^{2}(N+1)^{2}(N+2)^{3}}\right\}+C_{F} N_{f}\left\{-\frac{8\left(3 N^{2}+3 N+2\right)}{N(N+1)^{2}(N+2)} S_{1}\right. \\
& +\frac{32(N-1)}{(N-2)(N+1)(N+3)} S_{-2}+\frac{8 P_{9}(N)}{15(N-1)^{2} N^{2}(N+1)^{3}(N+2)^{2}(N+3)^{3}} \\
& \left.+\frac{32(-1)^{N} P_{10}(N)}{15(N-2)(N-1)^{2} N^{2}(N+1)^{3}(N+2)^{2}(N+3)^{3}}\right\} \\
& P_{6}(N)=2 N^{11}+41 N^{10}+226 N^{9}+556 N^{8}+963 N^{7}+2733 N^{6}+7160 N^{5}+8610 N^{4}+1969 N^{3} \\
& -2748 N^{2}-864 N-216 \\
& P_{7}(N)=1075 N^{12}+14390 N^{11}+73464 N^{10}+160740 N^{9}+35682 N^{8}-516984 N^{7}-979012 N^{6} \\
& -627068 N^{5}+84099 N^{4}+300258 N^{3}+119124 N^{2}-648 N+7776 \\
& P_{8}(N)=85 N^{12}+1130 N^{11}+5472 N^{10}+9300 N^{9}-13574 N^{8}-85432 N^{7}-149336 N^{6}
\end{aligned}
$$




$$
\begin{aligned}
& -114524 N^{5}-11383 N^{4}+44894 N^{3}+22992 N^{2}-4104 N-432 \\
P_{9}(N)= & 26 N^{9}+539 N^{8}+3244 N^{7}+8465 N^{6}+9342 N^{5}+841 N^{4}-5720 N^{3}-2193 N^{2} \\
& +2484 N+1404 \\
P_{10}(N)= & N^{10}-13 N^{9}-39 N^{8}+222 N^{7}+1132 N^{6}+1787 N^{5}+913 N^{4}+392 N^{3}+645 N^{2} \\
& -324 N-108
\end{aligned}
$$

The other Wilson coefficients have a similar structure, cf. [220,221].

At $O\left(a_{s}^{3}\right)$ a series of moments for the Wilson coefficients of the structure functions was calculated in [55] 58]. The complete expressions were computed in [61] and combinations of charged current structure functions in [222]. In Figure 10 the relative size of the QCD corrections to two Wilson coefficients is illustrated comparing the corrections $O\left(a_{s}\right)$ to $O\left(a_{s}^{3}\right)$. In the flavor non-singlet case the corrections for the Wilson coefficients at NNLO are larger than in case of the anomalous dimension for large values of $N$, resp. in the large $x$ region.
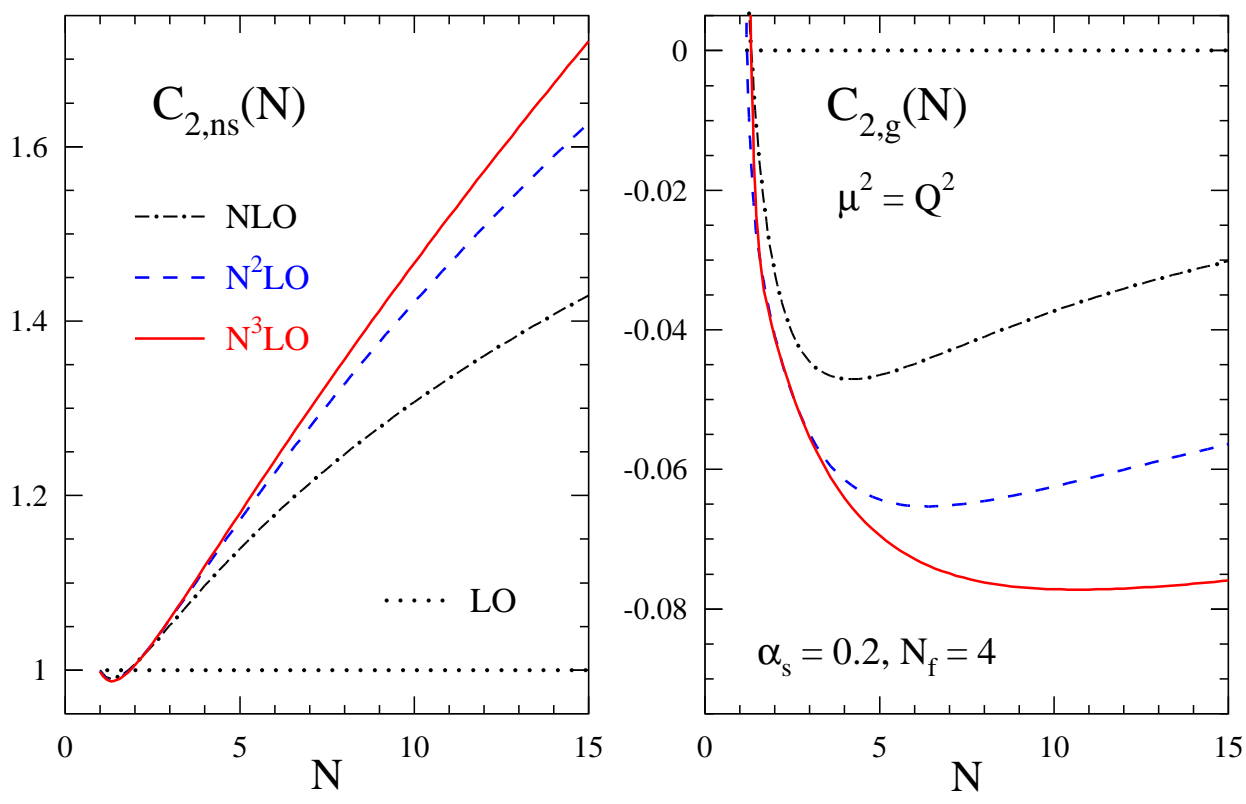

Figure 10: The perturbative expansion of the non-singlet (left) and gluon (right) $N$-space coefficient functions for $F_{2} / x$ at $\alpha_{s}=0.2, N_{f}=4$. $O\left(\alpha_{s}\right)$ dash-dotted lines, $O\left(\alpha_{s}^{2}\right)$ dashed lines, $O\left(\alpha_{s}^{3}\right)$ full lines. In the non-singlet case LO corresponds to $\alpha_{s}^{0}$, From [61], ( ${ }^{(2005)}$ by Elsevier Science.

The massless Wilson coefficient at $O\left(a_{s}^{3}\right)$ can be represented in terms of harmonic sums up to weight $\mathrm{w}=6$, resp. harmonic polylogarithms in $z$-space. The corresponding expressions are very large. Structural simplifications can be obtained applying algebraic [223] and structural relations [196] of these quantities. The Wilson coefficients obey difference equations of order $\sim 35$ and degree $\sim 1000$, which can be found in principle determining somewhat more than 5000 moments, [220]. The corresponding difference equations can be solved with the package Sigma [76].

\section{QED and Electro-weak Radiative Corrections to Deep- Inelastic Scattering}

The QED radiative corrections to the deep-inelastic scattering cross sections become rather large in part of the kinematic region due to logarithmic terms of $O\left(\alpha \ln \left(Q^{2} / m_{l}^{2}\right)\right)$, with $m_{l}$ the charged lepton 
mass. The corrections in case of $e^{ \pm} N$ are larger than for $\mu^{ \pm} N$ scattering. They have to be known precisely since their unfolding is usually performed for the scattering cross section itself prior to the QCD analyses of the deep-inelastic structure functions.

A first dedicated calculation of the QED radiative corrections to deep inelastic $e^{ \pm} N$ scattering was performed by Mo and Tsai [224] and applied in the analysis of the MIT-SLAC experiments, [15, 16]. Later calculations were carried out in [225, 226] for $l^{ \pm} N$ scattering.

The detailed knowledge of QED and electroweak radiative corrections was of special importance also for the measurements of the electroweak parameters in deep inelastic $\nu(\bar{\nu}) N$ scattering. Early calculations were carried out in [227]. More recent calculations have been performed in [228] in relation to the NuTeV anomaly [229]. The QED bremsstrahlung corrections apply to the lepton lines and the incoming quark line, since the corrections to the inclusive hadronic final state vanish according to the Kinoshita-Lee-Nauenberg theorem 230. In 231 the leading logarithmic corrections in $O(\alpha)$ were calculated, suggesting first to absorb the quarkonic QED corrections into the scaling violations of the quark distributions.

With the advent of HERA the radiative corrections were partly recalculated and dedicated calculations for deep inelastic neutral and charged current $e^{ \pm} p$ scattering were carried out by different groups, also using a variety techniques, $[84,90,226,232,250$. These approaches include both semi-analytic calculations [84, 90, 226, 234 247, 250] and calculations based on Monte Carlo methods [232, 233, 248, 249]. The virtual electro-weak 1-loop corrections to neutral and charged current deep-inelastic scattering were calculated in Refs. [232, 233, 235, 236]. The inclusive bremsstrahlung corrections are often presented integrating over the phase space of the emitted photons or lepton pairs, which can be performed analytically [234-243].

Dominant contributions to the QED radiative corrections may be obtained using leading log (LLA) techniques [84,226, 231, 238 242,244]. This approach, which is based on the factorization of (collinear) fermion mass singularities, allows to determine the terms $\propto \alpha \ln \left(Q^{2} / m_{f}^{2}\right)$ in a straightforward way for different settings of the measured kinematic variables. Also higher order terms were calculated within this approach [84,244.

The first order terms are described by:

$$
\frac{d^{2} \sigma^{\text {ini(fin),1loop }}}{d x d y}=\frac{\alpha}{2 \pi} L_{e} \int_{0}^{1} d z P_{e e}^{(0)}(z)\left\{\left.\theta\left(z-z_{0}\right) \mathcal{J}\left(x, y, Q^{2}\right) \frac{d^{2} \sigma^{0}}{d x d y}\right|_{x=\hat{x}, y=\hat{y}, S=\hat{S}}-\frac{d^{2} \sigma^{0}}{d x d y}\right\}
$$

where $P_{i j}^{(0)}(z)$ denote the leading order QED splitting functions with $i, j \in\{e, \gamma\}$. They are obtained from the QCD splitting functions given in Sect. 7 setting $C_{A}=0, C_{F}=1, T_{F}=1$. The scale of the correction is set by the logarithm

$$
L_{e}=\ln \frac{Q^{2}}{m_{e}^{2}}-1
$$

The second order corrections $\mathcal{O}\left(\left(\alpha L_{e}\right)^{2}\right)$ are:

$$
\begin{aligned}
& \frac{d^{2} \sigma^{l, 2 l o o p}}{d x d y}=\left[\frac{\alpha}{2 \pi} L_{e}\right]^{2} \int_{0}^{1} d z P_{e e}^{(2,1)}(z)\left\{\left.\theta\left(z-z_{0}\right) \mathcal{J}(x, y, z) \frac{d^{2} \sigma^{0}}{d x d y}\right|_{x=\hat{x}, y=\hat{y}, S=\hat{S}}-\frac{d^{2} \sigma^{0}}{d x d y}\right\} \\
& +\left.\left(\frac{\alpha}{2 \pi}\right)^{2} \int_{z_{0}}^{1} d z\left\{L_{e}^{2} P_{e e}^{(2,2)}(z)+L_{e} \sum_{f=l, q} \ln \frac{Q^{2}}{m_{f}^{2}} P_{e e, f}^{(2,3)}(z)\right\} \mathcal{J}(x, y, z) \frac{d^{2} \sigma^{0}}{d x d y}\right|_{x=\hat{x}, y=\hat{y}, S=\hat{S}}
\end{aligned}
$$




\begin{tabular}{|c|c|c|c|c|}
\hline \multicolumn{5}{|c|}{ Initial State Radiation $\hat{S}=z S$} \\
\hline Kinematics & $\hat{Q}^{2}$ & $\hat{y}$ & $\hat{z_{0}}$ & $\mathcal{J}(x, y, z)$ \\
\hline leptonic variables & $z Q_{l}^{2}$ & $\frac{z+y_{l}-1}{z}$ & $\frac{1-y_{l}}{1-x_{1} u_{l}}$ & $\frac{y_{l}}{z+y_{l}-1}$ \\
\hline mixed variables & $z Q_{l}^{2}$ & $\frac{\tilde{y} J B}{z}$ & $\begin{array}{c}1-x_{l} y_{l} \\
y_{J B}\end{array}$ & $\begin{array}{c}z+y_{l}-1 \\
1\end{array}$ \\
\hline hadronic variables & $Q_{h}^{2}$ & $\frac{\tilde{y} h}{z}$ & $\begin{array}{c}9 J B \\
y_{h}\end{array}$ & $\frac{1}{z}$ \\
\hline JB variables & $\underline{Q_{J B}^{2}\left(1-y_{J B}\right) z}$ & $\underline{y_{J B}}$ & $y_{J B}$ & $1-y_{J B}$ \\
\hline double anole method & $\begin{array}{c}\overline{z-y_{J B}} \\
z^{2} O^{2}\end{array}$ & $\begin{array}{c}z \\
u_{D+4}\end{array}$ & $\overline{1-x_{J B}\left(1-y_{J B}\right)}$ & $\overline{z-y_{J B}}$ \\
\hline$\theta_{1}$ & $O^{2} z\left(z-y_{J B}\right)$ & $\begin{array}{l}9 D A \\
y_{J B}\end{array}$ & 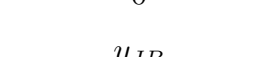 & $z-y_{J B}$ \\
\hline $\begin{array}{l}l, y_{J B} \\
\Sigma \text { method }\end{array}$ & $Q_{\theta y} \frac{1-y_{J B}}{Q_{\triangle}^{2}}$ & $\begin{array}{l}z \\
y_{\Sigma}\end{array}$ & $\begin{array}{c}y_{J B} \\
x_{\Sigma}\end{array}$ & $\begin{array}{l}1-y_{J B} \\
\underline{1}\end{array}$ \\
\hline$e \Sigma$ method & $z Q_{l}^{2}$ & $z y_{e \Sigma}$ & $x_{\Sigma}$ & $\stackrel{z}{1}$ \\
\hline \multicolumn{5}{|c|}{ Final State Radiation $\hat{S}=S$} \\
\hline leptonic variables & $\frac{Q_{l}^{2}}{\gamma}$ & $\frac{z+y_{l}-1}{s}$ & $1-y_{l}\left(1-x_{l}\right)$ & $\frac{y_{l}}{z\left(z+y_{l}-1\right)}$ \\
\hline mixed variables & $\frac{Q_{l}^{2}}{z}$ & $y_{J B}$ & $x_{m}$ & $\frac{1}{z}$ \\
\hline$\Sigma$ method & $Q_{\Sigma}^{2} \frac{\left(1-\tilde{y}_{\Sigma}(1-z)\right)}{z^{2}}$ & $\frac{y_{\Sigma} z}{1-y_{\Sigma}(1-z)}$ & $z_{0}^{\Sigma, f}$ & $\frac{1}{z^{2}}$ \\
\hline$e \Sigma$ method & $\frac{Q_{l}^{\tilde{2}}}{z}$ & $\frac{y_{e \Sigma} z^{2}}{\left(1-y_{\Sigma}(1-z)\right)^{2}}$ & $z_{0}^{\Sigma, f}$ & $\frac{1+y_{e \Sigma}(1-z)}{\left(1-y_{e \Sigma}(1-z)\right) z}$ \\
\hline
\end{tabular}

Table 1: Scaling properties of various sets of kinematic variables for leptonic initial and final state radiation, cf. [90].

This notion reproduces the soft photon terms of complete calculations in leptonic variables (cf. e.g. 90]). The shifted variables $\hat{x}, \hat{y}$, the threshold $z_{0}$ and the Jacobian $\mathcal{J}$ depend on the choice of the external kinematic variables, see Table 1, $S$ denotes the cms energy and the hats refer to variables in the sub-system.

Here the different second order splitting kernels are given by

$$
\begin{aligned}
P_{e e}^{(2,1)}(z) & =\frac{1}{2}\left[P_{e e}^{(0)} \otimes P_{e e}^{(0)}\right](z)=\frac{1+z^{2}}{1-z}\left[2 \ln (1-z)-\ln z+\frac{3}{2}\right]+\frac{1}{2}(1+z) \ln z-(1-z), \\
P_{e e}^{(2,2)}(z) & =\frac{1}{2}\left[P_{e \gamma}^{(0)} \otimes P_{\gamma e}^{(0)}\right](z) \equiv(1+z) \ln z+\frac{1}{2}(1-z)+\frac{2}{3} \frac{1}{z}\left(1-z^{3}\right) \\
P_{e e, f}^{(2,3)}(z) & =N_{c}(f) Q_{f}^{2} \frac{1}{3} P_{e e}^{(0)}(z) \theta\left(1-z-\frac{2 m_{f}}{E_{e}}\right)
\end{aligned}
$$

denoting double-photon radiation, scattering of a fermion into a fermion by a collinear photon, and collinear fermion pair production. Here, $m_{f}$ is the mass of the produced fermion, $Q_{f}$ its charge, $N_{c}(f)=$ 3 for quarks, $N_{c}(f)=1$ for leptons, respectively, and $\otimes$ denotes the Mellin-convolution (3.19). The soft-photon exponentiation is performed solving the non-singlet evolution equation in the range $z \rightarrow 1$ analytically, cf. e.g. [177]. Since the terms up to $\mathcal{O}\left(\alpha^{2}\right)$ were taken into account in Eq. (9.2) already the corresponding contributions have to be subtracted. One obtains [84]:

$$
\frac{d^{2} \sigma^{(>2, s o f t)}}{d x d y}=\int_{0}^{1} d z P_{e e}^{(>2)}\left(z, Q^{2}\right)\left\{\left.\theta\left(z-z_{0}\right) \mathcal{J}(x, y, z) \frac{d^{2} \sigma^{(0)}}{d x d y}\right|_{x=\hat{x}, y=\hat{y}, S=\hat{S}}-\frac{d^{2} \sigma^{(0)}}{d x d y}\right\}
$$

with

$$
P_{e e}^{>2}\left(z, Q^{2}\right)=\zeta(1-z)^{\zeta-1} \frac{\exp \left[\frac{1}{2} \zeta\left(\frac{3}{2}-2 \gamma_{E}\right)\right]}{\Gamma(1+\zeta)},-\frac{\alpha}{2 \pi} L_{e} \frac{2}{1-z}\left\{1+\frac{\alpha}{2 \pi} L_{e}\left[\frac{11}{6}+2 \ln (1-z)\right]\right\}
$$


with $\zeta=-3 \ln \left[1-(\alpha / 3 \pi) L_{e}\right]$.
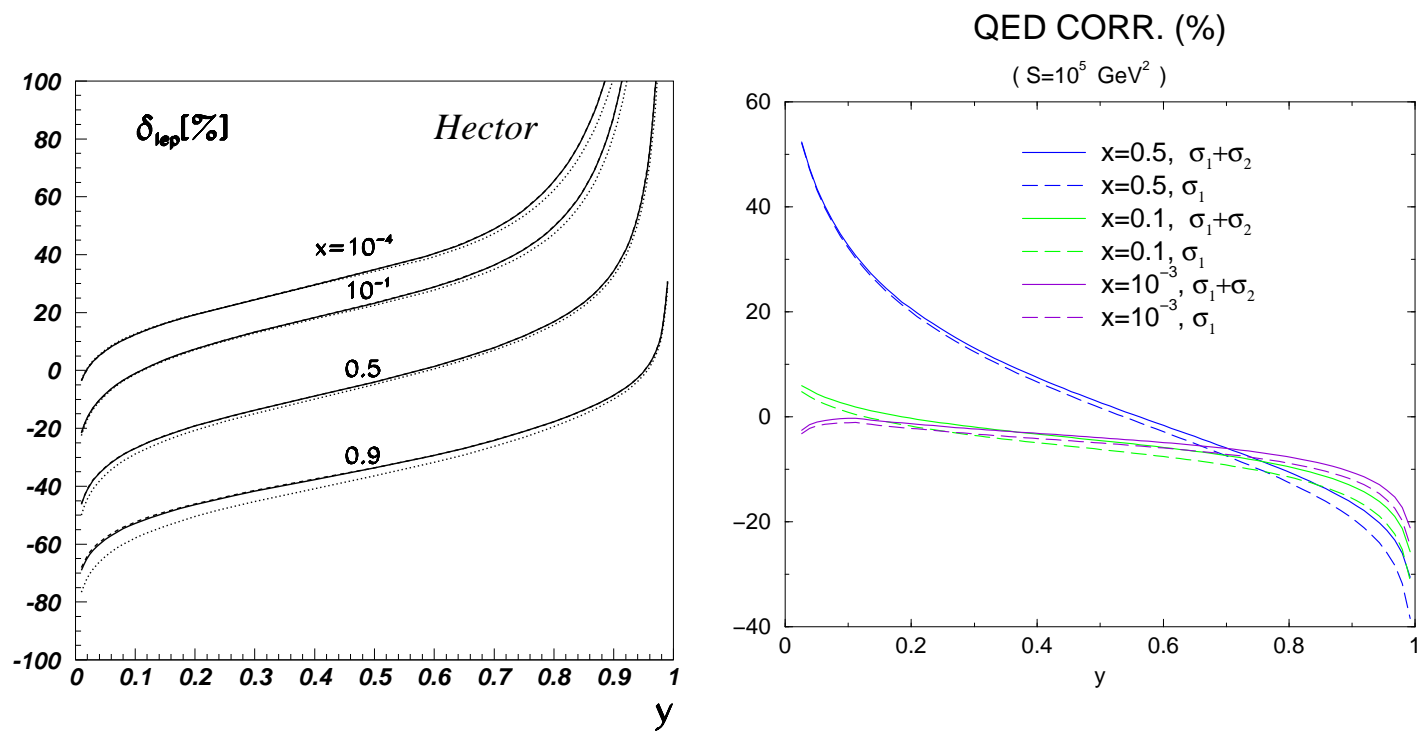

Figure 11: a) Radiative corrections in leptonic variables in per cent for $E_{e}=26.8 \mathrm{GeV}, E_{p}=820 \mathrm{GeV}$. Dotted lines: $O(\alpha)$, dashed lines: $O\left(\alpha^{2}\right)$, solid lines: in addition soft photon exponentiation, from [90], (C)(1995) by Elsevier Science; b) Radiative corrections in mixed variables in per cent for $E_{e}=26.8 \mathrm{GeV}, E_{p}=820 \mathrm{GeV}$. The lines left from above to below correspond to $x=0.5,0.1,0.001$ in consecutive order. Full lines $O(\alpha)+O\left(\alpha^{2} L^{2}\right)+O\left(\alpha^{2} L\right)$; dashed lines: $O(\alpha)$; Figure by courtesy of $\mathrm{H}$. Kawamura.

Finally, there is also a fermion conversion term in $\mathcal{O}\left(\alpha^{2} L_{e}^{2}\right), P\left(z, Q^{2} ; e^{-} \rightarrow e^{+}\right)=\left(\frac{\alpha}{2 \pi}\right)^{2} L_{e}^{2} P_{e e}^{(2,2)}(z)$, [84].

In Figure 11 we illustrate the size of QED radiative corrections for two types of the measurement of the kinematic variables in the case of neutral current deep inelastic scattering. In the first case the variables $x$ and $Q^{2}$ are measured from the scattered lepton only, while in the second case mixed variables, cf. Table 1, are applied, resulting into corrections of different type. The radiative corrections for leptonic variables become very large at small $x$ and high $y$. Choosing the double-angle method, one obtains corrections which behave rather flat in $y$, cf. [84]. The different methods to calculate the QED radiative corrections have been compared in $\mathcal{O}(\alpha)$ for a variety of kinematic measurements and are well understood. In 2nd order so far only LLA results are available [84, 244 for the full set of external kinematic variables studied by the HERA experiments. Only in the case of mixed variables also the $O\left(\alpha^{2} L\right)$ corrections were calculated [251].

In the region in which the virtuality $Q^{2}$ of the exchanged photon becomes very small the observed final state consists of a $p_{\perp}$-balanced electron-photon pair, with little hadronic activity near the beampipe. This contribution is called Compton peak and has been studied first in [224]. It is given by

$$
\frac{d^{2} \sigma^{C}}{d x_{l} d y_{l}}=\frac{\alpha^{3}}{x_{l} S}\left[1+\left(1-y_{l}\right)^{2}\right] \ln \left(\frac{Q_{l}^{2}}{M_{N}^{2}}\right) \int_{x_{l}}^{1} \frac{d z}{z^{2}} \frac{z^{2}+\left(x_{l}-z\right)^{2}}{x_{l}\left(1-y_{l}\right)} \sum_{f}\left[q_{f}\left(z, Q_{l}^{2}\right)+\bar{q}_{f}\left(z, Q_{l}^{2}\right)\right]
$$

cf. 239] for leptonic variables. The scale setting is the same as being used in [234] referring to the kinematic approach in which the hadronic structure is dealt with inclusively. A more refined expression than (9.8) was derived in Ref. [250]. LLA higher order corrections are easily obtained applying the corresponding radiators. The process was described using the parton model in [238] regulating the collinear singularity by finite light quark masses. 
In the initial calculations of the QED corrections to deep-inelastic scattering based on the parton model quark lines were treated as fermion lines with mass $m_{q}$ in the on-shell scheme [232, 233, 235, 236]. This led to logarithmic corrections of $O\left(\alpha e_{q}^{2} \ln \left(Q^{2} / m_{q}^{2}\right)\right)$ behaving flat in $y$ and growing with $x$ to $\sim 7-12 \%$ for $x=0.9 \ldots 0.99$. Here $e_{q}$ denotes the light quark's charge. The correct way in treating these contributions is to absorb them into the scaling violations of the partons along with the QCD corrections. At leading order one obtains the radiator

$$
\alpha_{s}\left(\mu^{2}\right)\left[1+e_{q}^{2} \frac{\alpha\left(\mu^{2}\right)}{C_{F} \alpha_{s}\left(\mu^{2}\right)}\right] P_{q q}^{0}(z) \ln \left(\frac{\mu^{2}}{m_{q}^{2}}\right) .
$$

In this way the correction becomes independent of the values of the light quark masses, cf. [231,239,243]. The overall effect is bounded by $<1.5 \%$ at HERA and hard to be resolved [252]. Later numerical studies of the resummation of the leading order QED effect were performed in [253].

The integral leptonic QED corrections to polarized deep-inelastic $l^{ \pm} N$ scattering, using the approach of form factors, have been calculated in [254], also presenting the leading log results. Here also the effect of the exchange of electro-weak gauge bosons was included, which is of relevance at high energy leptonnucleon colliders with polarized targets. Leading log results were given in [255]. Monte Carlo codes for the QED corrections in case of polarized deep-inelastic were designed in [256].

The well-known resummation of the soft corrections [177] to all orders in $O\left((\alpha L)^{k}\right)$ has been extended including the hard corrections to $O\left((\alpha L)^{3}\right)$ in [257, 258], to $O\left((\alpha L)^{5}\right)$ in the flavor non-singlet case in [259, 260], in the unpolarized flavor singlet case in [261], and in the polarized singlet case in [260]. These corrections are universal and can be applied as well for other scattering cross sections. For cms energies up to the $\mathrm{TeV}$ range these corrections are sufficient. However, depending on the process corrections to next-to-leading log may be needed.

Another higher order correction is due to small- $x$ resummation in case of QED. These are the resummed non-singlet corrections of $O\left(\left(\alpha \log ^{2}(x)\right)^{l}\right)$, cf. [262].

\section{Heavy Flavor Wilson Coefficients}

The heavy flavor contributions to the deep-inelastic structure functions are rather large in the unpolarized case for smaller values of $x$. Due to the different scaling violations of the massless and massive contributions to the structure functions the exact knowledge of the heavy flavor Wilson coefficients is important. At leading order they were calculated in the unpolarized case in [263] and in the polarized case in [214,264,265]. They are given by :

$$
\begin{aligned}
H_{g, F_{2}}^{(1)}\left(z, \frac{m^{2}}{Q^{2}}\right)= & 8 T_{F}\left\{v\left[-\frac{1}{2}+\left(4+\frac{2 m^{2}}{Q^{2}}\right) z(1-z)\right]+\left[-\frac{1}{2}+z(1-z)+2 \frac{m^{2}}{Q^{2}} z(3 z-1)+4 \frac{m^{4}}{Q^{4}} z^{2}\right]\right. \\
& \left.\times \ln \left(\frac{1-v}{1+v}\right)\right\} \\
H_{g, F_{L}}^{(1)}\left(z, \frac{m^{2}}{Q^{2}}\right)= & 16 T_{F}\left\{v z(1-z)+2 \frac{m^{2}}{Q^{2}} z^{2} \ln \left(\frac{1-v}{1+v}\right)\right\} \\
H_{g, g_{1}}^{(1)}\left(z, \frac{m^{2}}{Q^{2}}\right)= & 4 T_{F}\left\{v(3-4 z)+(1-2 z) \ln \left(\frac{1-v}{1+v}\right)\right\}
\end{aligned}
$$

with $v=\sqrt{1-4 m^{2} z /\left(Q^{2}(1-z)\right)}$ and $m$ the heavy quark mass. The heavy flavor contribution to the structure functions at $\mathrm{LO}$ is given by

$$
F_{2, L}^{Q \bar{Q}}\left(x, Q^{2}, m^{2}\right)=e_{Q}^{2} a_{s} \int_{a x}^{1} \frac{d z}{z} H_{g, F_{2, L}}^{(1)}\left(\frac{x}{z}, \frac{m^{2}}{Q^{2}}\right) G\left(z, Q^{2}\right), \quad a=1+\frac{4 m^{2}}{Q^{2}},
$$


and analogously for $g_{1}^{Q \bar{Q}}\left(x, Q^{2}, m^{2}\right)$. In the polarized case the heavy-flavor contributions to $g_{1}\left(x, Q^{2}\right)$ and $g_{2}\left(x, Q^{2}\right)$ show an oscillatory behavior [265] since the first moment of $H_{g, g_{1}}^{(1)}$ vanishes and $H_{g, g_{2}}^{(1)}$ obeys a Wandzura-Wilczek relation [266]. In the unpolarized case the heavy-flavor Wilson coefficients have been calculated at NLO in semi-analytic form in [65, 66] 6] A detailed proof of hard-scattering factorization including heavy quarks was given in [268.

In the region $Q^{2} \gg m^{2}$ the heavy-flavor Wilson coefficients factorize into massive operator matrix elements with massless external lines 7 and the corresponding massless Wilson coefficients [71]. For the structure function $F_{2}\left(x, Q^{2}\right)$ this representation holds at the $1 \%$ level for $Q^{2} \gtrsim 10 \mathrm{~m}^{2}$ [71], while for $F_{L}\left(x, Q^{2}\right)$ this is valid only at much higher scales $Q^{2} \gtrsim 800 \mathrm{~m}^{2}$. The results of [71] were confirmed in [270] and the $O\left(a_{s}^{2} \varepsilon\right)$ corrections were derived in [271]. In the polarized case the NLO heavy flavor Wilson coefficients were computed for the asymptotic region only [71,72]. At LO and NLO also heavy flavor corrections for the charged current case have been calculated [272].

Because of the high accuracy to which the structure function $F_{2}\left(x, Q^{2}\right)$ is measured, the heavy flavor Wilson coefficients have to be computed to NNLO, like the massless contributions. This is analytically possible using the method of massive operator matrix elements in the region $Q^{2} \gtrsim 10 \mathrm{~m}^{2}$. At 3-loop order the five Wilson coefficients $L_{q, i}^{\mathrm{NS}}, L_{q, i}^{\mathrm{PS}}, L_{g, i}^{\mathrm{S}}, H_{q, i}^{\mathrm{PS}}, H_{g, i}^{\mathrm{S}}$ contribute, cf. [67]. In [67] a series of Mellin moments has been calculated for the corresponding massive OMEs ranging up to $N=10, \ldots, 14$, depending on the process, applying the code MATAD [75]. First 3-loop results for general values of $N$ were derived in [194,195]. These calculations require completely different technologies. In 194 the Wilson coefficients $L_{q, i}^{\mathrm{PS}}$ and $L_{g, i}^{\mathrm{S}}$ were calculated completely. All logarithmic contributions $\propto \ln ^{k}\left(Q^{2} / m^{2}\right), k=1,2,3$ in the 3-loop case have been computed for general values of $N$ in [273]. The technology to calculate the ladder topologies is already available [274] and first results have been obtained for other more involved 3-loop topologies. Here codes like Sigma and HarmonicSums [76,275] are essential, which were developed further to cope with problems of this kind. In the asymptotic region also the 3-loop contributions for the heavy flavor Wilson coefficients to $F_{L}\left(x, Q^{2}\right)$ were calculated [276]. Furthermore, a series of moments of the massive OMEs contributing to transversity at $O\left(a_{s}^{3}\right)$ were computed in [277]. The heavy flavor Wilson coefficients at higher order can be expressed for the pole or running mass, which is implemented through the renormalization procedure, cf. Sect. 5, up to 3-loop order in [67]; for a phenomenological application at NLO see [278]. In the threshold region of heavy-quark pair production one may estimate higher order corrections using soft resummations. They have been studied in the unpolarized case in [279] and the polarized case in [280].

The above calculations were all performed in the fixed-flavor number scheme. As has been shown in [281, 282] this description is sufficient throughout the kinematic range at HERA for charm and bottom. Still one may consider to introduce a so-called variable flavor number scheme, in particular to match the universal contributions on the way a single massive quark is becoming light. Here one assumes that the transition always occurs for one heavy quark at the time. One problem in this context is that $m_{c}^{2} / m_{b}^{2} \approx 1 / 10$ and one cannot consider charm quarks as massless at $\mu^{2}=m_{b}^{2}$. The matching conditions for the flavor non-singlet and singlet parton distributions have been given at NLO in [283] and NNLO in [67] and the transition functions in [67, 71, 72, 283, 284, with first general $N$ results at NNLO in [194,195]. Different descriptions to interpolate between the heavy-quark threshold region and the asymptotic region are considered in the literature. As an example, in the BMSN-scheme [282, 283] the interpolation in $F_{2}\left(x, Q^{2}\right)$ is given by

$$
F_{2}^{Q \bar{Q}, \mathrm{BMSN}}\left(x, Q^{2}, N_{f}+1\right)=F_{2}^{Q \bar{Q}}\left(x, Q^{2}, N_{f}\right)+F_{2}^{Q \bar{Q}, \text { asymp }}\left(x, Q^{2}, N_{f}+1\right)-F_{2}^{Q \bar{Q}, \text { asymp }}\left(x, Q^{2}, N_{f}\right) .
$$

Here $F_{2}^{Q \bar{Q} \text {,asymp }}$ denotes the heavy flavor structure function in the limit of vanishing power corrections. Other schemes were proposed in [285 287]. The schemes differ w.r.t. the way how fast a massive

\footnotetext{
${ }^{6} \mathrm{~A}$ fast numerical implementation in Mellin-space has been given in [267].

${ }^{7}$ Massive OMEs with massive external lines have been dealt with in [269].
} 
quark becomes light. One may infer the correct behaviour from precision data. The matching scales are usually chosen by $\mu=m_{q}$. However, this scale is usually process dependent and may turn out to be much larger as has been pointed out in [288] comparing exact calculations with the interpolative description. In any case, the reordering of terms in the interpolation schemes, cf. [282, 283, 285 287, have to be compatible with the $\overline{\mathrm{MS}}$ scheme. Otherwise an according scheme-transformation has to be provided since in the PDF-fits usually $\overline{\mathrm{MS}}$ parton distribution functions are determined and nearly all partonic scattering cross sections in physical applications are computed in this scheme.

Finally we would like to mention that at 3-loop order also diagrams with two different fermion lines contribute [289]. Here the moments $N=2,4,6$ were calculated based on the code qexp [290]. These contributions are even universal. However, due to the fact the $m_{b}$ is not very much larger than $m_{c}$ one obtains here double-logarithmic contributions, which may not simply be absorbed into either the charm or the bottom distribution.

\section{Target Mass Corrections}

At low 4-momentum transfer $Q^{2}$ and large values of $x$ the nucleon mass $M$ modifies the scattering kinematics and therefore the nucleon structure functions. The corresponding relations have first been worked out by Nachtmann [291] in the unpolarized case 8 Besides the Bjorken variable $x$ the new variable

$$
\xi=\frac{2 x}{1+\sqrt{1+4 x^{2} M^{2} / Q^{2}}} \equiv \frac{2 x}{1+r}
$$

emerges. Here the mass of the quark in the scattering process is assumed to be the same, $m_{i}=m_{f}$. For $x \ll 1$ or $Q^{2} \rightarrow \infty$ both variables approach each other. The corrections for the different structure functions can be derived using the local operators as in the massless case, cf. Sect. 3, [293. In the following we discuss the corrections for deep inelastic scattering off unpolarized and polarized targets.

Let us define the integrals [93,293.

$$
\begin{array}{ll}
G_{1}(\xi)=\int_{\xi}^{1} \frac{d \xi^{\prime}}{\xi^{\prime}} F\left(\xi^{\prime}\right), & G_{2}(\xi)=\int_{\xi}^{1} \frac{d \xi^{\prime}}{\xi^{\prime}} \int_{\xi^{\prime}}^{1} \frac{d \xi^{\prime \prime}}{\xi^{\prime \prime}} F\left(\xi^{\prime \prime}\right), \quad G_{3}(\xi)=\int_{\xi}^{1} d \xi^{\prime} \int_{\xi^{\prime}}^{1} \frac{d \xi^{\prime \prime}}{\xi^{\prime \prime}} \int_{\xi^{\prime \prime \prime}}^{1} \frac{d \xi^{\prime \prime \prime}}{\xi^{\prime \prime \prime}} F\left(\xi^{\prime \prime \prime}\right), \\
H_{1}(\xi)=\int_{\xi}^{1} d \xi^{\prime} F\left(\xi^{\prime}\right), & H_{2}(\xi)=\int_{\xi}^{1} d \xi^{\prime} \int_{\xi^{\prime}}^{1} d \xi^{\prime \prime} F\left(\xi^{\prime \prime}\right)
\end{array}
$$

and the operators

$$
\begin{gathered}
O_{F_{1}}^{(1)}=\frac{x}{2 r}, \quad O_{F_{1}}^{(2)}=-\frac{M^{2}}{Q^{2}} x^{2} \frac{d}{d x} \frac{x}{\xi r}, \quad O_{F_{2}}=x^{2} \frac{d^{2}}{d x^{2}} \frac{x^{2}}{\xi^{2} r}, \quad O_{F_{3}}=-x \frac{d}{d x} \frac{x}{\xi r} \\
O_{g_{1}}=x \frac{d}{d x} x \frac{d}{d x} \frac{x}{r \xi}, \quad O_{g_{2}}=-x \frac{d^{2}}{d x^{2}} \frac{x^{2}}{r \xi}, \quad O_{g_{3}}=2 x^{2} \frac{d^{2}}{d x^{2}} \frac{x^{2}}{r \xi^{2}}, \quad O_{g_{4}}=-x^{2} \frac{d}{d x} x \frac{d^{2}}{d x^{2}} \frac{x^{2}}{r \xi^{2}}, \\
O_{g_{5}}^{(1)}=-x \frac{d}{d x} \frac{x}{r \xi}, \quad O_{g_{5}}^{(2)}=\frac{M^{2}}{Q^{2}} x^{2} \frac{d^{2}}{d x^{2}} \frac{x^{2}}{r \xi} .
\end{gathered}
$$

Here a generic function $F(\xi)$ has been introduced, which reproduces the corresponding structure function in the massless case containing the respective electro-weak couplings and parton content. As an example, the function $F$ in case of the unpolarized structure functions $F_{1}$ and $F_{2}$ is the same. The target mass

${ }^{8}$ The same method has been applied to the polarized case in [292]. 
corrections can now be written in the following compact form for the unpolarized structure functions $F_{1,2,3}$ and the polarized structure functions $g_{1, \ldots, 5}$ in the twist-2 approximation :

$$
\begin{array}{rlr}
F_{1}\left(x, Q^{2}\right) & =O_{F_{1}}^{(1)} F(\xi)+O_{F_{1}}^{(2)} H_{2}(\xi) & \\
F_{2}\left(x, Q^{2}\right) & =O_{F_{2}}^{(1)} H_{2}(\xi) & \\
F_{3}\left(x, Q^{2}\right) & =O_{F_{3}}^{(1)} H_{1}(\xi) & i=1,2 \\
g_{i}\left(x, Q^{2}\right) & =O_{g_{i}} G_{2}(\xi), & i=3,4 \\
g_{i}\left(x, Q^{2}\right) & =O_{g_{i}} G_{3}(\xi), & \\
g_{5}\left(x, Q^{2}\right) & =O_{g_{5}}^{(1)} G_{1}(\xi)+O_{g_{5}}^{(2)} G_{3}(\xi) . &
\end{array}
$$

These relations easily transform into the corresponding integral representations 9 For the implementation of the target mass corrections in Mellin-space one may refer to contour integral representations directly, see [93,293]. In the unpolarized case they are given by

$$
\begin{aligned}
& F_{1}\left(x, Q^{2}\right)=\frac{1}{2 \pi i} \int_{-i \infty}^{i \infty} d N x^{-N} \sum_{j=0}^{\infty}\left(\frac{M^{2}}{Q^{2}}\right)^{j}\left(\begin{array}{c}
N+j \\
j
\end{array}\right)\left[\frac{1}{2} a_{N+2 j}^{(2)}+x \frac{M^{2}}{Q^{2}} \frac{N a_{N+2 j+1}^{(2)}}{(N+2 j)(N+2 j+1)}\right], \\
& F_{2}\left(x, Q^{2}\right)=\frac{1}{2 \pi i} \int_{-i \infty}^{i \infty} d N x^{-N+1} \sum_{j=0}^{\infty}\left(\frac{M^{2}}{Q^{2}}\right)^{j}\left(\begin{array}{c}
N+j \\
j
\end{array}\right) \frac{N(N-1)}{(N+2 j)(N+2 j-1)} a_{N+2 j}^{(2)}, \quad[293] \\
& F_{3}\left(x, Q^{2}\right)=\frac{1}{2 \pi i} \int_{-i \infty}^{i \infty} d N x^{-N} \sum_{j=0}^{\infty}\left(\frac{M^{2}}{Q^{2}}\right)^{j}\left(\begin{array}{c}
N+j \\
j
\end{array}\right) \frac{N}{N+2 j} a_{N+2 j}^{(3)}
\end{aligned}
$$

with $a_{k}=\int_{0}^{1} d z z^{k} F(z)$. In the limit $M^{2} \rightarrow 0$ the structure functions $F_{1,3}$ contain one power in $x$ less than $F_{2}$. Note that the definition of the OMEs $a_{k}$ differ in the literature. The target mass corrections for the longitudinal structure function are obtained by [293]

$$
2 x F_{L}=r^{2} F_{2}-2 x F_{1} .
$$

The corresponding relations in the polarized case were given in [93. For lower values of $x<0.7$ and not too small values of $Q^{2} \gtrsim 1 \mathrm{GeV}^{2}$ the infinite series in (11.10 11.12) may be approximated using the first five terms to reach sufficient precision. In the large- $x$ domain convergence is reached using up to $\sim 250$ terms. This requires economic implementations of the OMEs $a_{k}$, changing during fits to data, for the respective complex values of $k$ along the integration contour, which has been given in [295] recently.

Target mass corrections lead to a violation of the Callan-Gross relation [20]. Furthermore, one may derive integral relations in the polarized case, cf. Sect. 15, In the limit $x \rightarrow 1$ the structure functions (11.4 11.9) do not vanish unlike the partonic functions $F(x)$. This has led to numerous discussions in the literature [296,297]. Effects in this region cannot be considered without of a careful study of higher twist effects. In [298] besides the usual target mass corrections contributions of the jet-function at large values of $x$ were considered to improve the description in this region. However, this approach has to be viewed in comparison with others w.r.t. a consequent twist expansion and the fact, that the whole hadronic final state Fock-space has to be summed over.

Let us mention that in [93] also the corresponding relations for the twist-3 contributions to the polarized structure functions were derived. The target mass corrections in case of twist-2 and 3, (11.7), were also derived in [299]. For non-forward and diffractive scattering they have been calculated in 300,301] and 302].

\footnotetext{
${ }^{9}$ We agree with the results of [293] up to the obvious typos in (4.19) and (4.22), cf. also [294].
} 


\section{Solution of the Evolution Equations and Parton Distribu- tion Functions}

In Mellin-space the singlet evolution equations can be solved analytically. We follow [303] and express the evolution equation (5.5) in terms of $a_{s}=\alpha_{s}\left(Q^{2}\right) / 4 \pi$. The r.h.s. is expanded in the coupling constant. The singlet evolution equation reads :

$$
\begin{aligned}
\frac{\partial \boldsymbol{q}\left(a_{s}, N\right)}{\partial a_{s}}= & \frac{a_{s} \boldsymbol{P}_{0}(N)+a_{s}^{2} \boldsymbol{P}_{1}(N)+a_{s}^{3} \boldsymbol{P}_{2}(N)+\ldots}{-a_{s}^{2} \beta_{0}-a_{s}^{3} \beta_{1}-a_{s}^{4} \beta_{2}-\ldots} \boldsymbol{q}\left(a_{s}, N\right)=-\frac{1}{\beta_{0} a_{s}}\left[\boldsymbol{P}_{0}(N)+a_{s}\left(\boldsymbol{P}_{1}(N)-\frac{\beta_{1}}{\beta_{0}} \boldsymbol{P}_{0}(N)\right)\right. \\
& \left.+a_{s}^{2}\left(\boldsymbol{P}_{2}(N)-\frac{\beta_{1}}{\beta_{0}} \boldsymbol{P}_{1}(N)+\left\{\left(\frac{\beta_{1}}{\beta_{0}}\right)^{2}-\frac{\beta_{2}}{\beta_{0}}\right\} \boldsymbol{P}_{0}(N)\right)+\ldots\right] \boldsymbol{q}\left(a_{s}, N\right) \\
= & -\frac{1}{a_{s}}\left[\boldsymbol{R}_{0}(N)+\sum_{k=1}^{\infty} a_{s}^{k} \boldsymbol{R}_{k}(N)\right] \boldsymbol{q}\left(a_{s}, N\right),
\end{aligned}
$$

with $\boldsymbol{R}_{0} \equiv \boldsymbol{P}_{0} / \beta_{0}, \boldsymbol{R}_{k} \equiv\left[\boldsymbol{P}_{k}-\sum_{i=1}^{k} \beta_{i} \boldsymbol{R}_{k-i}\right] / \beta_{0} 10$ Here $\beta_{k}, k \geq 0$ denote the expansion coefficients of the $\beta$-function and $\boldsymbol{P}_{k}, k \geq 0$ are the singlet matrices of the splitting functions and

$$
\boldsymbol{q}\left(a_{s}, N\right)=\left(\begin{array}{c}
\Sigma\left(a_{s}, N\right) \\
G\left(a_{s}, N\right)
\end{array}\right)
$$

with $\Sigma\left(a_{s}, N\right)=\sum_{i=1}^{N_{f}}\left[q\left(a_{s}, N\right)+\bar{q}\left(a_{s}, N\right)\right], G\left(a_{s}, N\right)$ the flavor singlet and gluon distributions.

One may obtain the evolution equations for the three flavor non-singlet cases by replacing the matrices in (12.1) by scalars. In the singlet case the matrices $\boldsymbol{R}_{k}$ do not commute in general. Firstly, the leading order solution is found by

$$
\boldsymbol{q}^{\mathrm{LO}}\left(a_{s}, N\right)=\left(a_{s} / a_{0}\right)^{-\boldsymbol{R}_{0}(N)} \boldsymbol{q}\left(a_{0}, N\right) \equiv \boldsymbol{L}\left(a_{s}, a_{0}, N\right) \boldsymbol{q}\left(a_{0}, N\right)
$$

with the starting distribution $\boldsymbol{q}\left(a_{0}, N\right)$ and

$$
\boldsymbol{L}\left(a_{s}, a_{0}, N\right)=\boldsymbol{e}_{-}(N)\left(a_{s} / a_{0}\right)^{-r_{-}(N)}+\boldsymbol{e}_{+}(N)\left(a_{s} / a_{0}\right)^{-r_{+}(N)} .
$$

The projectors $\boldsymbol{e}_{ \pm}$are

$$
\boldsymbol{e}_{ \pm}=\frac{1}{r_{ \pm}-r_{\mp}}\left[\boldsymbol{R}_{0}-r_{\mp} \boldsymbol{I}\right] ; \quad r_{ \pm}=\frac{1}{2 \beta_{0}}\left[P_{q q}^{(0)}+P_{g g}^{(0)} \pm \sqrt{\left(P_{q q}^{(0)}-P_{g g}^{(0)}\right)^{2}+4 P_{q g}^{(0)} P_{g q}^{(0)}}\right] .
$$

Here $r_{ \pm}$denote the leading order eigenvalues. The general solution is given by

$$
\begin{aligned}
\boldsymbol{q}\left(a_{s}, N\right) & =\boldsymbol{U}\left(a_{s}, N\right) \boldsymbol{L}\left(a_{s}, a_{0}, N\right) \boldsymbol{U}^{-1}\left(a_{0}, N\right) \boldsymbol{q}\left(a_{0}, N\right) \\
& =\left[1+\sum_{k=1}^{\infty} a_{s}^{k} \boldsymbol{U}_{k}(N)\right] \boldsymbol{L}\left(a_{s}, a_{0}, N\right)\left[1+\sum_{k=1}^{\infty} a_{0}^{k} \boldsymbol{U}_{k}(N)\right]^{-1} \boldsymbol{q}\left(a_{0}, N\right) .
\end{aligned}
$$

The matrices $\boldsymbol{U}_{k}$ obey

$$
\begin{aligned}
{\left[\boldsymbol{U}_{k}, \boldsymbol{R}_{0}\right] } & =\boldsymbol{R}_{k}+\sum_{i=1}^{k-1} \boldsymbol{R}_{k-i} \boldsymbol{U}_{i}+k \boldsymbol{U}_{k} \equiv \widetilde{\boldsymbol{R}}_{k}+k \boldsymbol{U}_{k}, k \geq 1 \\
\boldsymbol{U}_{k} & =-\frac{1}{k}\left[\boldsymbol{e}_{-} \widetilde{\boldsymbol{R}}_{k} \boldsymbol{e}_{-}+\boldsymbol{e}_{+} \widetilde{\boldsymbol{R}}_{k} \boldsymbol{e}_{+}\right]+\frac{\boldsymbol{e}_{+} \widetilde{\boldsymbol{R}}_{k} \boldsymbol{e}_{-}}{r_{-}-r_{+}-k}+\frac{\boldsymbol{e}_{-} \widetilde{\boldsymbol{R}}_{k} \boldsymbol{e}_{+}}{r_{+}-r_{-}-k} .
\end{aligned}
$$

\footnotetext{
${ }^{10}$ The solution up to $k=3$ was given in $[304$.
} 
Potential poles in $\boldsymbol{U}_{k}(N)$ are canceled by those in $\boldsymbol{U}^{-1}$ in (12.6). The perturbative solution in $\mathrm{N}^{k} \mathrm{LO}$ is obtained expanding (12.6) up to the $k$ th common power in $a_{s}$ and $a_{0}$ keeping $\boldsymbol{L}\left(a_{s}, a_{0}, N\right)$.

The different structure functions in $N$-space are represented by

$$
F_{l}\left(a_{s}, a_{0}, N\right)=\sum_{n=1}^{3} C_{n, l}^{\mathrm{NS}}\left(a_{s}, N\right) q_{n}^{\mathrm{NS}}\left(a_{s}, a_{0}, N\right)+C_{l}^{\mathrm{S}}\left(a_{s}, N\right) \Sigma\left(a_{s}, a_{0}, N\right)+C_{l}^{\mathrm{g}}\left(a_{s}, N\right) G\left(a_{s}, a_{0}, N\right),
$$

where $C_{n, l}^{\mathrm{NS}, \mathrm{S}, \mathrm{g}}$ denote the corresponding Wilson coefficients and $q_{n}^{\mathrm{NS}}$ are the flavor non-singlet distributions. In a consistent representation one also expands (12.8) in powers of $a_{s}, a_{0}$, similar to (12.6), matching the factorization scales. All these operations can be performed analytically.

To transform to $x$-space a single precise contour integral is carried out numerically around the singularities of the problem. Usually these are poles on the real axis for $\operatorname{Re}(\mathbf{z})<c$, with a given constant $c$. If small- $x$ resummations are included the singularities may be located in the complex plane, cf. Section 13, The contour integral is given by

$$
x f(x)=\frac{1}{\pi} \int_{0}^{\infty} d z \operatorname{Im}\left[e^{i \phi} x^{-C} f(N=C)\right],
$$

since usually $f^{*}(N)=f\left(N^{*}\right)$ is obeyed, [305], and $C=c+z e^{i \phi}$. The solution of the evolution equations in Mellin space allows for fast and very precise numerical implementations. In other approaches the evolution equations are solved in momentum-fraction space. Public codes for the solution of the evolution equations are e.g. QCD-Pegasus [306], Hoppet [307], QCDNUM [308], and Openqcdrad [309].

The distributions $q_{n}^{\mathrm{NS}}\left(a_{0}, N\right), \Sigma\left(a_{0}, N\right)$ and $G\left(a_{0}, N\right)$ are non-perturbative quantities, which have to be fitted to the world precision data on deep-inelastic scattering and suitable other hard scattering processes. Their shape is a prioiri unknown and the corresponding parameterizations, valid in the kinematic region to be analyzed, have to be found. One way to determine these shapes is to express the distribution functions in terms of orthogonal polynomials, which has been studied in detail in the past [310, 311]. The Laguerre polynomials $\mathrm{L}_{n}[\ln (1 / x)]$ converge fastest [311]. In general the number of orthogonal polynomials needed is too large compared to the possible amount of parameters which can be fitted from the data. One way out consists in designing shapes in terms of polynomials or related functions, which are suggested by the orthogonal polynomial analysis and extend these forms gradually. The NNPDF collaboration uses neural network techniques 312 to find the correct shape of the parton densities and to estimate their errors. Due to the finite experimental accuracy and the limited amount of data points available it seems that the present number of parameters cannot exceed 30-40 in the unpolarized case. In the polarized case this number is even lower. The errors of the statistical and systematic errors of the parton distribution functions can be determined in $\chi^{2}$-analyses.

MSTW 2008 NLO PDFs (68\% C.L.)
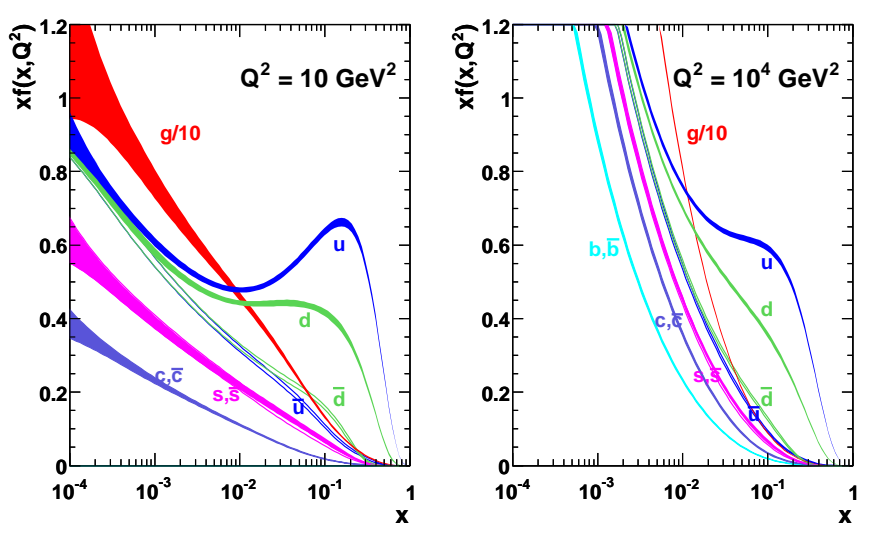

Figure 12: The MSTW 2008 NLO PDFs at $Q^{2}=10 \mathrm{GeV}^{2}$ and $10^{4} \mathrm{GeV}^{2}$; from [112], ((2009) by Springer Verlag. 
The valence quark distributions $u_{v}=u-\bar{u}$ and $d_{v}=d-\bar{d}$ are constrained by the following sum rules in case of the proton :

$$
\int_{0}^{1} d x u_{v}\left(x, Q^{2}\right)=2, \quad \int_{0}^{1} d x d_{v}\left(x, Q^{2}\right)=1 .
$$

Furthermore, assuming twist-2 dominance, the momentum sum rule reads :

$$
\int_{0}^{1} d x x\left[\Sigma\left(x, Q^{2}\right)+G\left(x, Q^{2}\right)\right]=1 .
$$

In the following we give a brief summary on the status reached for unpolarized 11 and polarized twist2 parton distribution functions (PDFs). They are obtained by the QCD analysis of the deep inelastic structure functions, supplemented by other hard scattering processes. The latter data sets mainly serve the purpose to resolve the flavor structure of the quarkonic sea and also might give better constraints on the gluon.
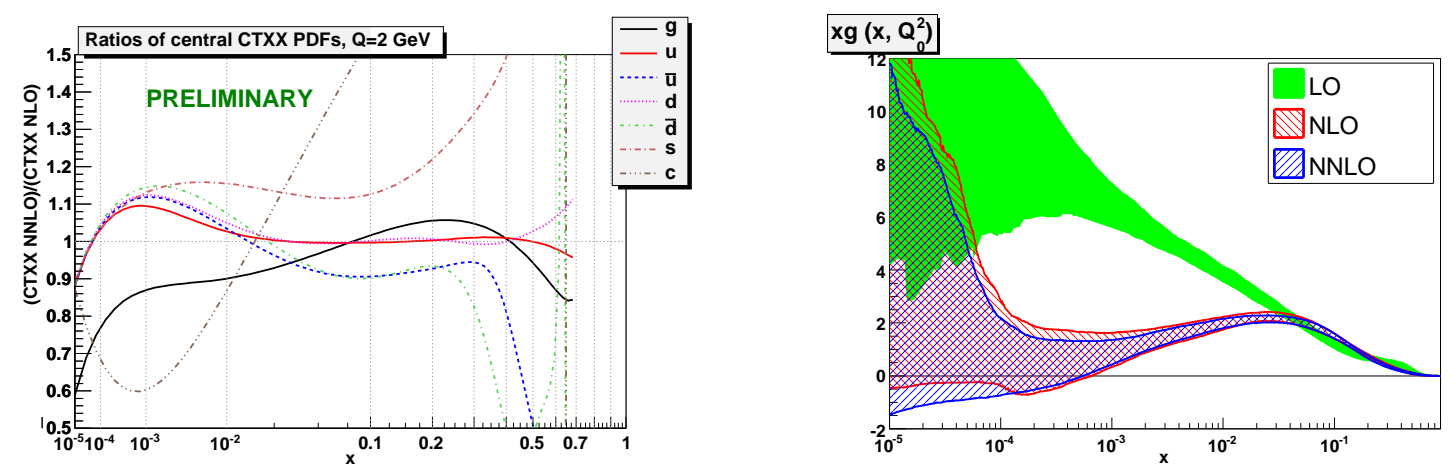

Figure 13: Left : Ratios of the PDFs at NNLO and NLO by CTEQ for $Q^{2}=4 \mathrm{GeV}^{2}$; courtesy P. Nadolsky. Right : Comparison of the gluon distribution by NNPDF2.1 at LO, NLO and NNLO at $Q^{2}=2 \mathrm{GeV}^{2}$; from [113], (2)(2012) by Elsevier Science.

\subsection{Unpolarized Nucleons}

Early QCD analyses of deep-inelastic structure functions have been performed starting in the late 1970ies in LO, and later in NLO, to derive first principal shapes of the parton densities, cf. [315 318. Analyses of the Dortmund group [316, 319], the MRS group [320], and the CTEQ (then Morfin-Tung) group [321] followed. In the first analyses the charm quark contributions were either treated as massless or at LO, since higher order corrections were not yet know.

At present the differential scattering cross sections of unpolarized deep-inelastic scattering in the massless case are known to NNLO and the heavy flavor corrections to NLO. The present highest order parton fits are based on this approximation. We note that some of the data contain significant higher twist contributions which have to be quantified, or, if possible for the corresponding reaction, cut away. Furthermore, target mass corrections have to be applied, since part of the scaling violations in the large $x$ and lower $Q^{2}$ region are due to these, cf. [199].

At present the six collaborations ABM [117], CTEQ [322], HERAPDF [323], JR [118], MSTW [112], and NNPDF [119] perform NNLO analyses, partly using different data sets. In Figure 12 an overview on the principal behaviour of the parton distributions as a function of $x$ are given for two typical scales

\footnotetext{
${ }^{11}$ For extensive recent reviews see [313, 314$]$.
} 
of $Q^{2}$ by MSTW at NLO. The distributions rise towards small values of $x$ and become steeper with growing values of $Q^{2}$.
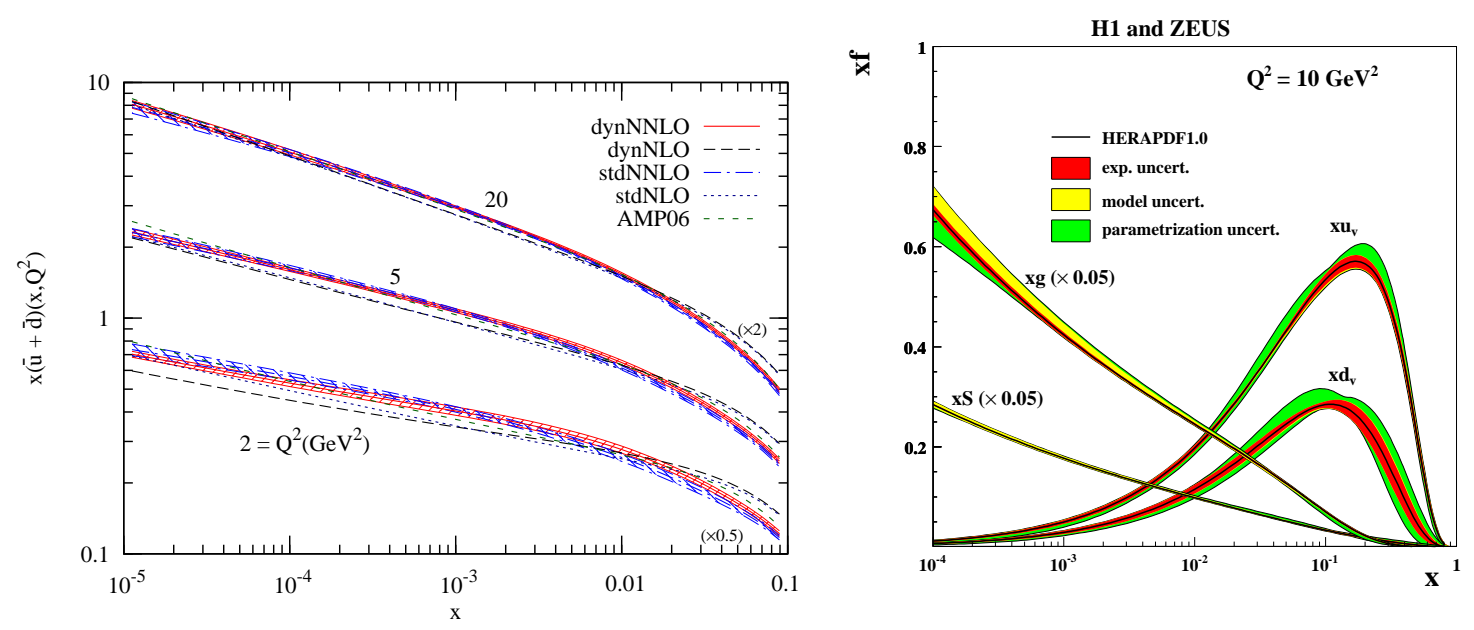

Figure 14: Left : Comparing the $1 \sigma$ error bands of the dynamical (dyn) and standard (std) NNLO gluon JR distributions [118] at small $x$ for various fixed values of $Q^{2}$. Note that $Q^{2}=2 \mathrm{GeV}^{2}$ is the input scale of the standard fit. The central NLO results are taken from [324] with uncertainties comparable to the ones shown for NNLO for $Q^{2}$ above $2 \mathrm{GeV}^{2}$. For comparison the 'standard' NNLO results of AMP06 [325] are shown as well. The results at $Q^{2}=2$ and $20 \mathrm{GeV}^{2}$ have been multiplied by 0.5 and 2, respectively, as indicated in the figure; from [118], ( $(2009)$ by the American Physical Society. Right: The parton distribution functions from HERAPDF1.0, $x u_{v}, x d_{v}, x S=2 x(\bar{U}+\bar{D}), x g$, at $Q^{2}=10 \mathrm{GeV}^{2}$. The gluon and sea distributions are scaled down by a factor 20 . The experimental, model and parameterization uncertainties are shown separately; from [104], ()(2009) by Elsevier Science.

Furthermore, the heavier flavors, charm and bottom, contribute above the corresponding thresholds. At large values of $x$ the up- and down-quark distributions show a different behaviour due to the valence quark contributions and the various sea quark contributions are of different size. In Figure 13 recent results of the CTEQ and NNPDF analyses are shown. The size of the ratios of the PDFs by CTEQ obtained in NNLO and NLO are compared at a low scale for the different partons showing a particular sensitivity in the region of small and large values of $x$. The gluon density is an important distribution for many processes at hadron colliders. A comparison is shown for the shapes obtained for $G\left(x, Q_{0}^{2}\right)$ in the NNPDF analysis from LO to NNLO. Here the gluon distribution is becoming lower at higher orders in the small $x$ region, with still a wide error band. In Figure 14 the evolution of the light sea-quark distributions of the JR NNLO analysis are shown in the small- $x$ region. Both the dynamical approach and the standard fit do agree rather well. The results of the HERAPDF 1.0 NLO analysis, which are based on data taken at HERA only, are shown also quantifying the parameterization uncertainty. It turns out, that the latter is of the size of the present experimental uncertainty. Figure 15 shows the $1 \sigma$ error bands obtained in the ABM11 analysis and compares the different massless parton distribution to the results found by the latest JR, MSTW, NNPDF analyses at the scale $\mu=2 \mathrm{GeV}$. While the valence distributions and the sum of the up and down sea-quarks do agree rather well in all the four fits, there are still important differences in the gluon distribution at lower values of $x$. In particular the gluon distribution of MSTW is taking low values in the small $x$ region, and eventually becomes negative unlike in all the other analyses. There are also differences in the $d_{s}-u_{s}=\bar{d}-\bar{u}$ distribution and for the strange quark density at medium values of $x$.

In Figure 16] different lattice determinations of the moment $\int_{0}^{1} d x\left[x\left(u_{v}-d_{v}\right)-x(\bar{u}-\bar{d})\right]$ are compared with the corresponding value obtained from PDF fits, see also [326]. Very similar values are obtained by the different fitting groups, cf. [117]. There is still a difference between the lattice results and the 
value from the PDF analyses, despite lower pion masses are used in the present lattice simulations, which has to be understood further. A determination of the gluon momentum with lattice methods has recently been performed in [327]. For a review on the status of the calculations of PDF-moments with lattice methods see [328].

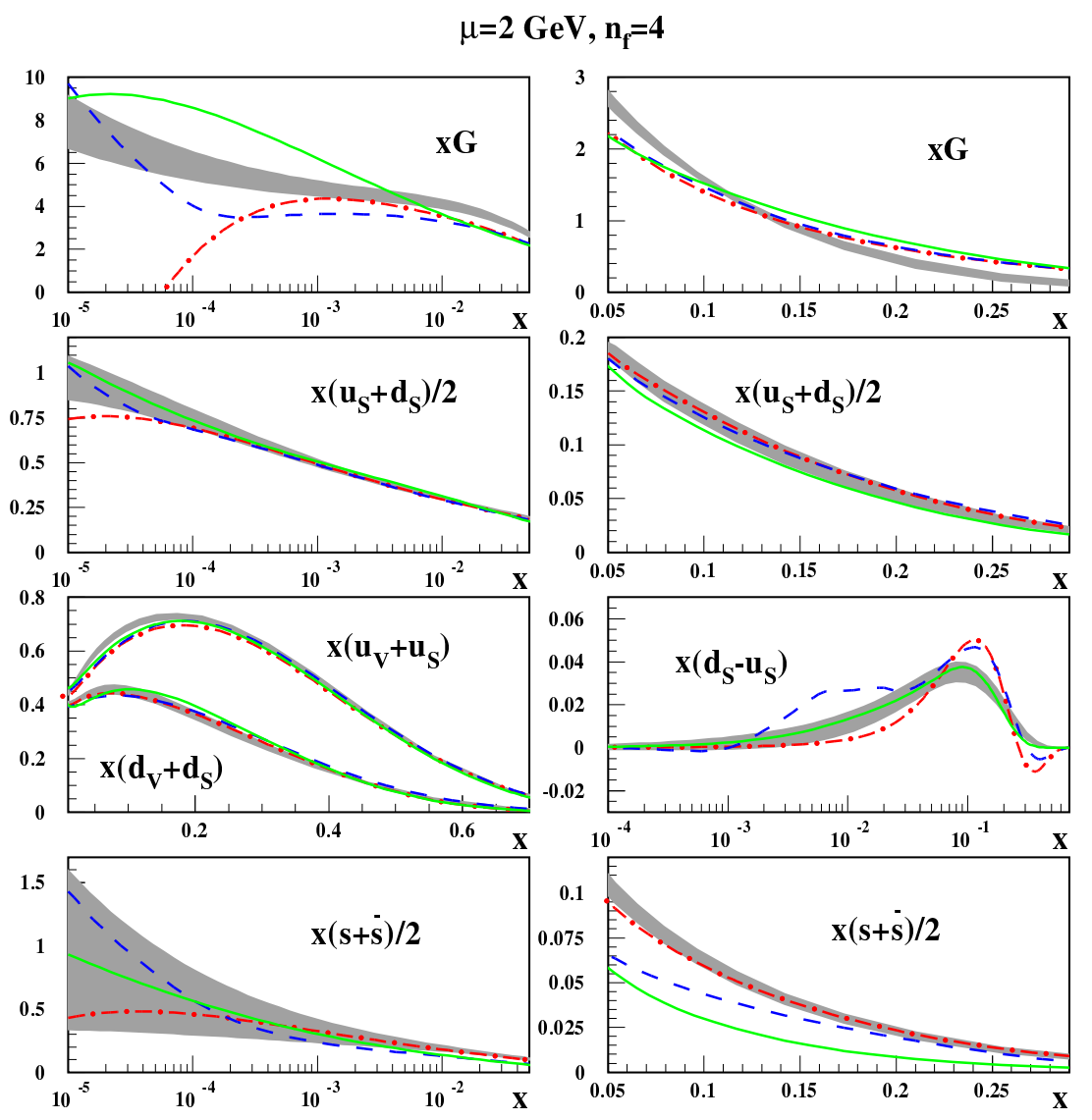

Figure 15: The $1 \sigma$ error band for the 4-flavor NNLO ABM11 PDFs at the scale of $\mu=2 \mathrm{GeV}$ versus $x$ obtained in [117] (shaded area) compared with the ones obtained by other groups. Solid lines: JR09 [118], dashed dots: MSTW [112], dashes: NN21 [119]; from [117].

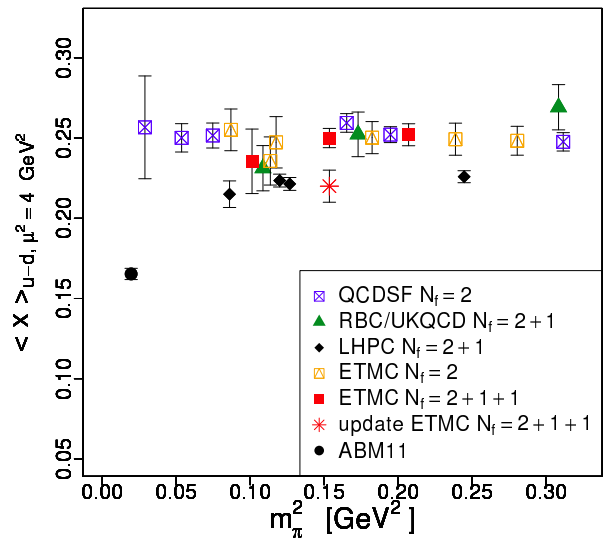

Figure 16: Comparison of lattice computations for the second moment of the non-singlet distribution as a function of the pion mass $m_{\pi}$ with the result of ABM11 [117] along with the uncertainties of the respective measurement; from [117], by courtesy of V. Drach. 

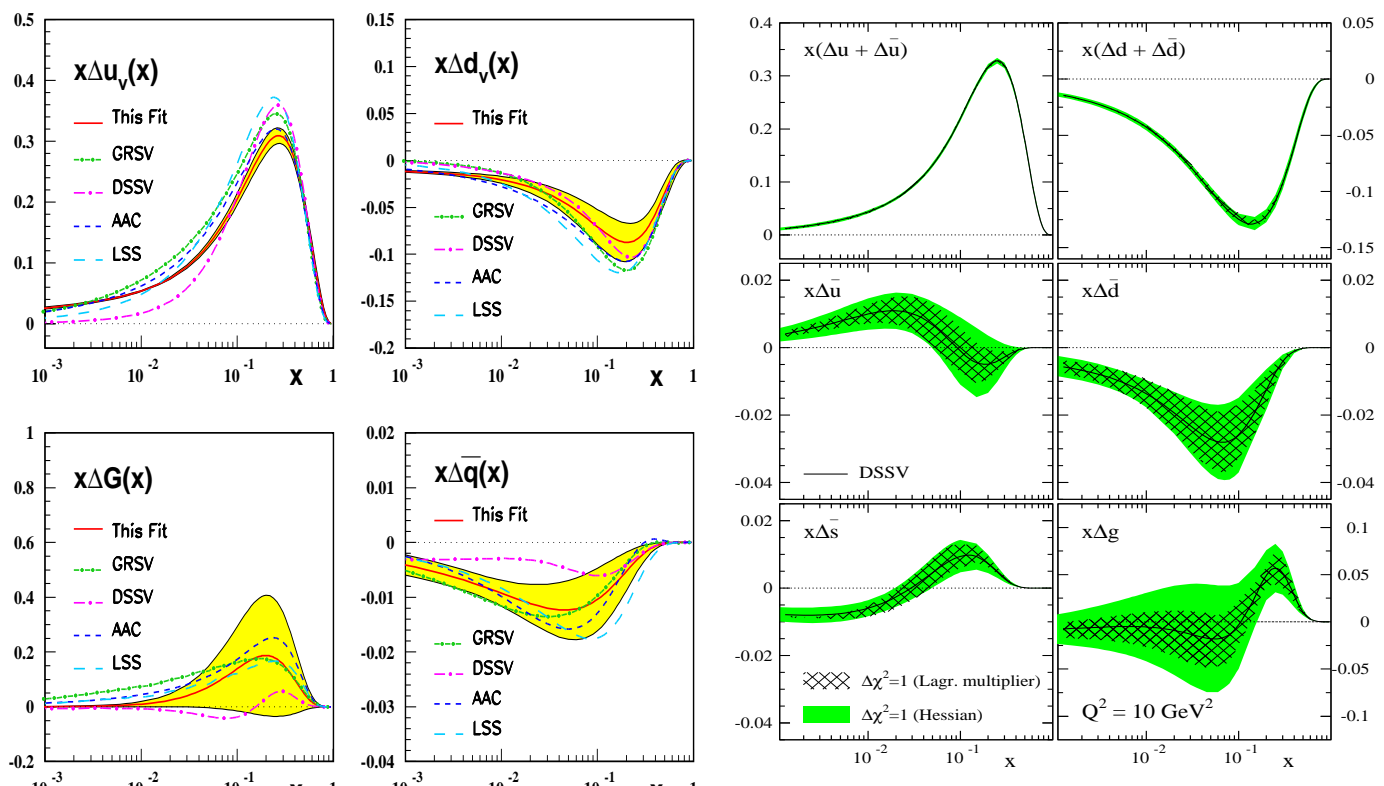

Figure 17: Left: The NLO polarized parton BB distributions [329] at the input scale $Q_{0}^{2}=4.0 \mathrm{GeV}^{2}$ (solid line) compared to results obtained by GRSV [330] (dashed-dotted line), DSSV (long dashed-dotted line) [331], AAC (dashed line) [332], and LSS (long dashed line) [333]. The shaded areas represent the fully correlated $1 \sigma$ error bands calculated by Gaussian error propagation; from [329], ( ${ }^{(2010)}$ by Elsevier Science. Right : The polarized DSSV PDFs [331] of the proton at $Q^{2}=10 \mathrm{GeV}^{2}$ in the $\overline{\mathrm{MS}}$ scheme, along with their $\Delta \chi^{2}=1$ uncertainty bands computed with Lagrange multipliers and the improved Hessian approach; from [331], (C)(2009) by the American Physical Society.

\subsection{Polarized Nucleons}

Early determinations of the polarized parton densities were carried out at the beginning of the 1990ies at LO under a series of model assumptions, see e.g. [334,335]. With the advent of the NLO polarized anomalous dimension NLO analyses were carried out [215, 333, 334, 336, 337]; cf. also [338, 339]. In these analyses $S U(3)_{F}$ symmetry was assumed for the sea quarks. In Figure 17 the results of different polarized PDF-analyses are compared (left panel). The errors on these distributions are larger than in the unpolarized case, with a basic agreement in the results for the valence distributions. For the sea-quark and gluon distribution the errors are larger and there is a stronger variation of the current predictions, widely within the present errors. We note also other approaches based on statistical distributions [340], which allow for a very efficient modeling.

To resolve the flavor dependence of the sea-quarks data on semi-inclusive measurements, as e.g. [341, are used. Furthermore, data on photo- and electro-production of hadrons and charm and proton-proton collisions at RHIC can be used, along with the structure function data. A recent analysis based on these data was carried out in [331]. The semi-inclusive data were also used in the analysis [342]. The polarized PDFs of [331] are shown in Figure 17 (right panel) and allow, yet with larger errors, to derive different shapes for the polarized sea quarks. The gluon distribution is found to be much lower than in the case of the analysis of only the deep-inelastic structure function data.

\section{$12.3 \alpha_{s}\left(M_{Z}^{2}\right)$}

The strong coupling constant can be determined as one parameter in the QCD-analysis of the deep inelastic world data along with the parameters of the non-perturbative parton distributions at a given 
scale $Q_{0}^{2}$. In the following we will compare the results on $\alpha_{s}\left(M_{Z}^{2}\right)$ in different NNLO analyses. The results are summarized in Table 2, cf. [117].

\begin{tabular}{|l|l|l|}
\hline & \multicolumn{1}{|c|}{$\alpha_{s}\left(M_{Z}^{2}\right)$} & \\
\hline BBG & $0.1134_{-0.0021}^{+0.0019}$ & valence analysis, NNLO [199] \\
GRS & 0.112 & valence analysis, NNLO [343] \\
ABKM & $0.1135 \pm 0.0014$ & HQ: FFNS $n_{f}=3$ [282] \\
ABKM & $0.1129 \pm 0.0014$ & HQ: BSMN-approach [282] \\
JR & $0.1124 \pm 0.0020$ & dynamical approach [118] \\
JR & $0.1158 \pm 0.0035$ & standard fit [118] \\
ABM11 & $0.1134 \pm 0.0011$ & [117] \\
MSTW & $0.1171 \pm 0.0014$ & 344] \\
NN21 & $0.1173 \pm 0.0007$ & 345] \\
CT10 & $0.118 \pm 0.005$ & 346] \\
\hline BBG & $0.1141+0.0020$ & valence analysis, $\mathrm{N}^{3} \mathrm{LO}\left(^{*}\right)[199]$ \\
\hline world average & $0.1183 \pm 0.0010$ & 347] (2011) \\
\hline
\end{tabular}

Table 2: Summary of recent NNLO QCD analyses of the DIS world data.

There are two valence analyses [199,343], effectively limited to the region of $x \gtrsim 0.3$. In [199] an effective $\mathrm{N}^{3} \mathrm{LO}$ fit has been performed, including the 3-loop Wilson coefficient, noting that the effect of the 4-loop non-singlet anomalous dimension is very small. The other analyses cover the whole kinematic range. In the valence analyses and [117, 118, 282] very similar values of $\alpha_{s}\left(M_{Z}^{2}\right)$ are obtained, which agree within their errors. The way in which the heavy flavor corrections are treated implies a systematic error of 0.0006 . The $\mathrm{N}^{3} \mathrm{LO}$ value is well compatible with the results obtained at NNLO. These values are lower than the current world average [347]. Larger values are found in the MSTW [344] and NNPDF analyses [345]. A preliminary value has been reported by CTEQ [346] at NNLO, yet with a rather large error. In Ref. [117] a detailed analysis has been performed comparing the above results also with respect to the pulls given by the different data sets used. Despite the final $\alpha_{s}\left(M_{Z}^{2}\right)$ values of MSTW and NNPDF are quite similar, there are still significant differences in the pulls. The Tevatron jet data do not cause the larger values. It rather seems that the response of the MSTW and NNPDF fits to the SLAC and also the HERA data is causing this difference, which needs to be investigated further.

In different other reactions larger values of $\alpha_{s}\left(M_{Z}^{2}\right)$ were found. This applies to the analysis of the 3-jet rate with $\alpha_{s}\left(M_{Z}^{2}\right)=0.1175 \pm 0.0025$ [348] and inclusive $Z$-decay yielding $\alpha_{s}\left(M_{Z}^{2}\right)=0.1189 \pm 0.0026$ [349,350]. This also applies to the $\alpha_{s}\left(M_{Z}^{2}\right)$ values measured for hadronic $\tau$-decays, cf. [347]. On the other hand, the analysis of thrust in $e^{+} e^{-}$annihilation led to $\alpha_{s}\left(M_{Z}^{2}\right)=0.1153 \pm 0.0017 \pm 0.0023$ [351], resp., $\alpha_{s}\left(M_{Z}^{2}\right)=0.1135 \pm 0.0011 \pm 0.0006$ [352]. In a NLO analysis of $e^{+} e^{-} \rightarrow 5$ jets $\alpha_{s}\left(M_{Z}^{2}\right)=0.1156_{-0.0034}^{+0.0041}$ was obtained. There are also first results on the measurements of $\alpha_{s}\left(M_{Z}^{2}\right)$ with including dynamical fermions from the lattice [353. Very recently a NLO analysis of the ATLAS jet data resulted in $\alpha_{s}\left(M_{Z}^{2}\right) \sim 0.1156$ with a larger error [354]. Similar results are obtained in a multi-jet analysis of $e^{+} e^{-}$ data [355]. Usually the values of $\alpha_{s}\left(M_{Z}^{2}\right)$ obtained at NNLO are lower than those at NLO. In various classes of high energy reactions partly larger and lower values of $\alpha_{s}\left(M_{Z}^{2}\right)$ are obtained at present. This is due to systematics sources, which have to be understood better in the future. Further clarification can be obtained from better hard scattering data. Here the LHC jet data will be of importance in the near future. 


\section{Small $x$ Resummations}

Approaching very small values of $x$ at a fixed virtuality $Q^{2}$ the criteria for the parton model, Section 4 , are no longer valid and new phenomena are expected to contribute. The small $x$ region was probed at HERA to values of $x \sim 10^{-4}$ at $Q^{2}=10 \mathrm{GeV}^{2}$ and at LHC values of $x \sim 10^{-6}$ at $Q^{2}=10^{2} \mathrm{GeV}^{2}$ can be reached. The potentially new effects will influence the size and scaling violations of the structure functions both in the unpolarized and polarized case. To which extent these phenomena can be dealt with using perturbative methods and resummations based on them, is not finally clear at present. Two main extensions of the perturbative approach based on fixed order perturbative QCD were proposed: i) the linear BFKL small- $x$ resummation [356] of large logarithms $\left(\alpha_{s}\left(N_{c} / \pi\right)\right)^{k}(1 / x) \ln ^{k}(1 / x) / k$ !, and ii) saturation models with highly non-linear gluo-dynamics, cf. [357] related to Glauber-models [358] 12 In both approaches elements known from the parton picture are used. Since perturbative kernels are evaluated it has to be clarified whether factorization holds. In case of the saturation corrections this also applies to multi-parton states. Due to the connection between the non-perturbative distributions and the perturbative kernels, usually in terms of a convolution, both effects at small and larger values of $x$ will contribute. In the following we will not cover the saturation models and related theoretical developments in nucleon-nucleus and nucleus-nucleus scattering, being an interesting broad topic in its own right. We refer to recent detailed surveys, as e.g. given in [138,361], and will discuss the effects due to BFKL-type resummations.

The resummation of the leading small $x$ terms [356] are often confronted to those in $\ln \left(Q^{2}\right)$, stating that the first ones results from the strong ordering $x_{1} \gg \ldots x_{i} \gg x_{i+1} \ldots$ and the second stem from strong ordering in the transverse momentum $k_{\perp, 1} \ll \ldots k_{\perp, i} \ll k_{\perp, i+1} \ldots$ along a ladder 13 Note that this is a gauge-dependent statement and requires to use effective vertices in case of the first approach. The second case refers to fixed-order perturbation theory using the renormalization group, as outlined in the previous sections. In the first approach one would calculate in a more systematic way the scale-invariant contributions to the evolution equation (5.5) in the massless case setting $\beta=0$, i.e. considering the strong coupling as const. One obtains

$$
E_{k}^{n}\left(\mu^{2}\right)=E_{k}^{n}\left(\mu_{0}^{2}\right)\left(\frac{\mu^{2}}{\mu_{0}^{2}}\right)^{\frac{1}{2}\left(\gamma_{O_{k}}-n \gamma_{\Phi}\right)} .
$$

The scale invariant part of the anomalous dimension has the representation

$$
\gamma_{O_{k}}-n \gamma_{\Phi}=\sum_{l=1}^{\infty} \gamma_{O}^{(l)} a_{s}^{l}
$$

and exponentiates to all orders. The representation (13.1) applies also for higher order resummations under the above requirements. To treat running coupling effects one has to systematically account for scale-invariance breaking effects. Furthermore, to form observables, the small- $x$ resummed Wilson coefficients have to be calculated as well.

Both in the flavor non-singlet and singlet cases for scattering off unpolarized and polarized targets the leading order small- $x$ resummations were derived in form of evolution kernels. They seem to correspond to a resummation of the leading order anomalous dimensions, as the comparison in the known orders show, because the corresponding singularity in the Wilson coefficients turns out to be one order lower [59,60, 363 .

\footnotetext{
${ }^{12}$ For early estimates of the transition line between the perturbative and non-perturbative domain and the onset the possible of saturation effects see [359, 360.

${ }^{13}$ One may study this process under a more general point of view and consider angularly ordered emissions covering both the above cases [362], which allows for interesting applications through Monte Carlo studies. Whereas this unified treatment is possible at $\mathrm{LO}$, higher order corrections cannot be cast into this form in general.
} 


\subsection{The Non-singlet and Polarized Singlet Contribution}

The most singular contributions to the Mellin transforms of the structure-function evolution kernels at all orders in $a$ stem from the poles at $N=0$ in each individual order. In the flavor non-singlet case the kernels $K^{ \pm}$can be obtained from the positive and negative signature amplitudes studied in [364] for QCD via

$$
\mathcal{M}\left[K_{x \rightarrow 0}^{ \pm}(a)\right](N) \equiv \int_{0}^{1} d x x^{N-1} K_{x \rightarrow 0}^{ \pm}(x, a) \equiv-\frac{1}{2} \Gamma_{x \rightarrow 0}^{ \pm}(N, a),
$$

with

$$
\begin{gathered}
\Gamma_{x \rightarrow 0}^{+}(N, a)=-N\left\{1-\sqrt{1-\frac{8 a C_{F}}{N^{2}}}\right\} \\
\Gamma_{x \rightarrow 0}^{-}(N, a)=-N\left\{1-\sqrt{1-\frac{8 a C_{F}}{N^{2}}\left[1-\frac{8 a N_{c}}{N} \frac{d}{d N} \ln \left(e^{z^{2} / 4} D_{-1 /\left[2 N_{c}^{2}\right]}(z)\right)\right]}\right\},
\end{gathered}
$$

where $z=N / \sqrt{2 N_{c} a}, D_{p}(z)$ denotes the function of the parabolic cylinder, and $N_{c}=3$ in case of QCD.

The LO small $x$ evolution kernels in the case of the polarized singlet evolution were derived in [365]. The resummed splitting function is given by

$$
\boldsymbol{P}\left(x, a_{s}\right) \equiv \sum_{l=0}^{\infty} \boldsymbol{P}_{x \rightarrow 0}^{(l)} a_{s}^{l+1} \log ^{2 l} x=\frac{1}{8 \pi^{2}} \mathcal{M}^{-1}\left[\boldsymbol{F}_{0}\left(N, a_{s}\right)\right](x) .
$$

The matrix valued function $\boldsymbol{F}_{0}\left(N, a_{s}\right)$ is obtained as the solution of

$$
\boldsymbol{F}_{0}\left(N, a_{s}\right)=16 \pi^{2} \frac{a_{s}}{N}\left(\begin{array}{cc}
C_{F} & -2 T_{R} N_{f} \\
2 C_{F} & 4 C_{A}
\end{array}\right)-\frac{8 a_{s}}{N^{2}} \boldsymbol{F}_{8}\left(N, a_{s}\right)\left(\begin{array}{cc}
C_{F} & 0 \\
0 & C_{A}
\end{array}\right)+\frac{1}{8 \pi^{2}} \frac{1}{N} \boldsymbol{F}_{0}^{2}\left(N, a_{s}\right)
$$

with

$$
\boldsymbol{F}_{8}\left(N, a_{s}\right)=16 \pi^{2} \frac{a_{s}}{N} \boldsymbol{M}_{8}=\left(\begin{array}{cc}
C_{F}-C_{A} / 2 & -T_{R} N_{f} \\
C_{A} & 2 C_{A}
\end{array}\right)+\frac{2 a_{s}}{N} C_{A} \frac{d}{d N} \boldsymbol{F}_{8}\left(N, a_{s}\right)+\frac{1}{8 \pi^{2}} \frac{1}{N} \boldsymbol{F}_{8}^{2}\left(N, a_{s}\right) .
$$

Eq. (13.6) obeys [366]

$$
P_{q g}^{(l)} /\left(T_{R} N_{f}\right)=-P_{g q}^{(l)} / C_{F}
$$

to all orders, where $T_{R}=1 / 2$ and $N_{f}$ denotes the number of flavors. The leading contributions of the fixed order results in LO and NLO $(\overline{\mathrm{MS}})$ are correctly described. In the supersymmetric limit $C_{A}=C_{F}=N_{f}=1, T_{R}=1 / 2$ the relations

$$
P_{q q}^{(l)}+P_{g q}^{(l)}=P_{q g}^{(l)}+P_{g g}^{(l)}
$$

are obeyed for all $l$ and Eq. (13.6) can be given in a simple analytic form [366]. It is evident from Eqs. (13.4 13.6) that the poles at $N=0$ being present in the individual orders are resummed into branch cuts, which usually exhibit a milder singularity.

To perform numerical studies it is interesting to consider the effect of less singular terms to estimate the stability of the leading order resummed terms. In case of the fixed-order anomalous dimensions they are known and one may try Ansätze like
(a) $\Gamma\left(N, a_{s}\right) \rightarrow \Gamma\left(N, a_{s}\right)-\Gamma\left(1, a_{s}\right)$
(b) $\Gamma\left(N, a_{s}\right) \rightarrow \Gamma\left(N, a_{s}\right)(1-N)$
(c) $\Gamma\left(N, a_{s}\right) \rightarrow \Gamma\left(N, a_{s}\right)(1-N)^{2}$
(d) $\Gamma\left(N, a_{s}\right) \rightarrow \Gamma\left(N, a_{s}\right)\left(1-2 N+N^{3}\right)$, 
cf. 303]. It turns out that the corrections to the flavor non-singlet contributions are numerically very small [363]. Larger corrections are obtained in the polarized singlet case, see Figure 18. However, here sub-leading terms e.g. of the type $(13.11(b))$ do nearly completely cancel these contributions again. Comparable results were obtained in [367]. Large effects as anticipated in [368] are not confirmed.
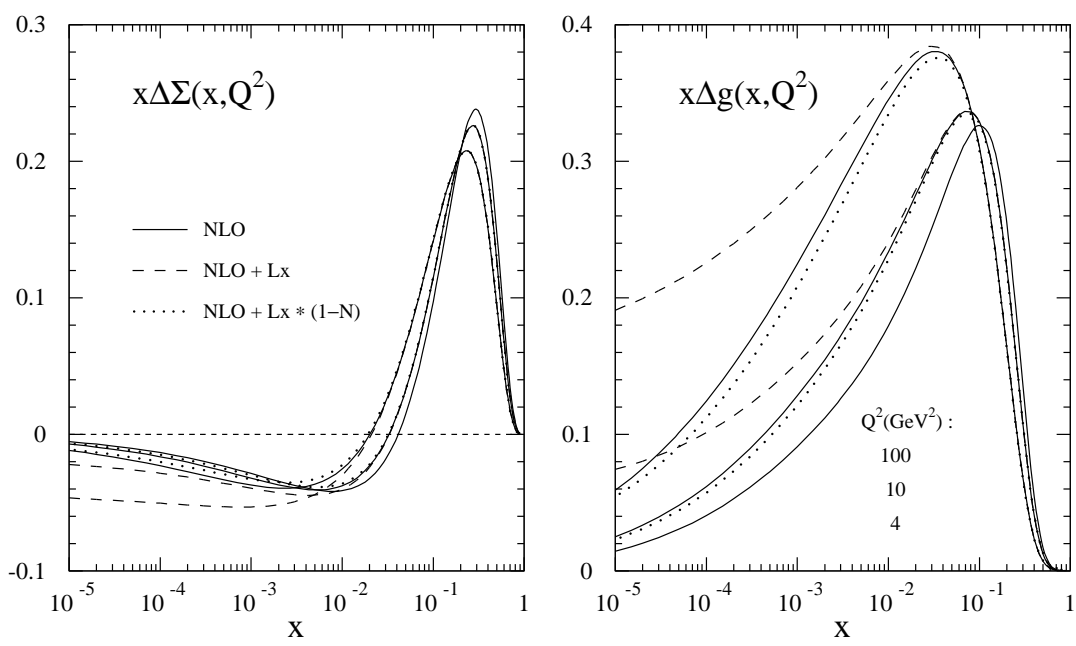

Figure 18: The $Q^{2}$ evolution of the polarized quark singlet and gluon momentum distributions evolving from $Q_{0}^{2}=4 \mathrm{GeV}^{2}$; from [366], ()(1996) by Elsevier Science.
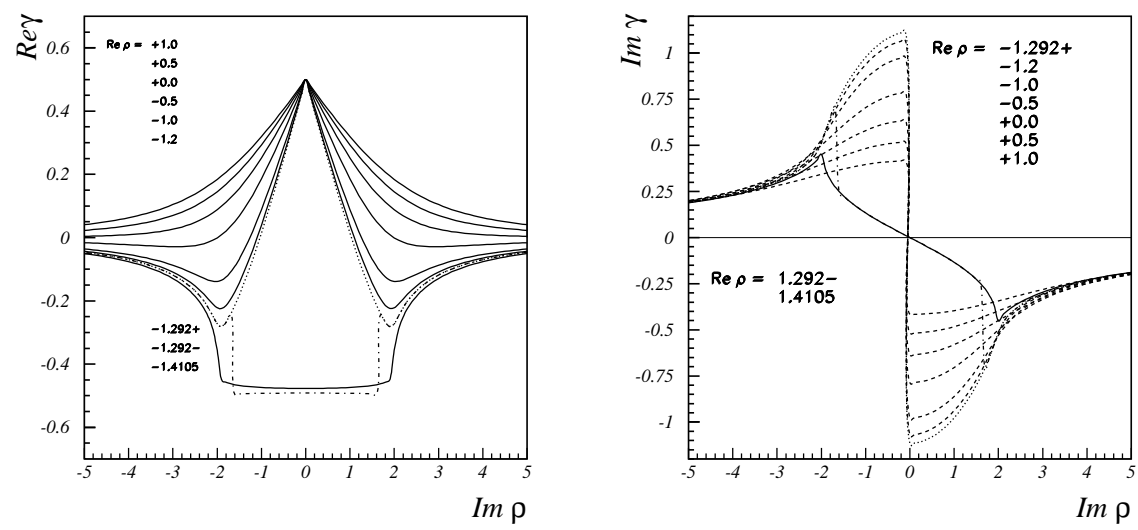

Figure 19: Real and imaginary part of the LO BFKL anomalous dimension; from [303, ()(1998) by the American Physical Society.

\subsection{Unpolarized Singlet Distributions}

The LO resummation for the evolution kernel of the unpolarized singlet distributions was derived in [356]. It was shown in [369] that the eigenvalue

$$
(N-1)=\frac{\alpha_{s} N_{c}}{\pi} \chi_{0}\left(\gamma_{L}\right) \equiv \frac{\alpha_{s} N_{c}}{\pi}\left[2 \psi(1)-\psi\left(\gamma_{L}\right)-\psi\left(1-\gamma_{L}\right)\right]
$$

represents the LO resummed gluon-gluon anomalous dimension $\gamma_{L}=\gamma_{g g}^{(0)}\left(N, a_{s}\right)$. The resummed LO gluon-quark anomalous dimension is given by $\gamma_{g q}^{(0)}\left(N, a_{s}\right)=\left(C_{F} / C_{A}\right) \gamma_{L}$ and the quarkonic terms do not 
contribute in $O\left[\left(a_{s} /(N-1)\right)^{l}\right]$. Eq. (13.12) can be solved iteratively demanding $\gamma_{L}\left(N, a_{s}\right) \rightarrow \bar{\alpha}_{s} /(N-1)$ as $|N| \rightarrow \infty$ for $N \in \mathbb{C}$, which selects the physical branch of the resummed anomalous dimension,

$$
\gamma_{L} \equiv \gamma_{g g, 0}\left(N, \alpha_{s}\right)=\frac{\bar{\alpha}_{s}}{N-1}\left\{1+2 \sum_{l=1}^{\infty} \zeta_{2 l+1} \gamma_{g g, 0}^{2 l+1}\left(N, \alpha_{s}\right)\right\}
$$

Here we rewrite $\bar{\alpha}_{s}=N_{c} \alpha_{s} / \pi \cdot \gamma_{L}$ has the serial representation

$$
\gamma_{g g, 0}\left(N, \alpha_{s}\right)=\frac{\bar{\alpha}_{s}}{N-1}+2 \zeta_{3}\left(\frac{\bar{\alpha}_{s}}{N-1}\right)^{4}+2 \zeta_{5}\left(\frac{\bar{\alpha}_{s}}{N-1}\right)^{6}+12 \zeta_{3}^{2}\left(\frac{\bar{\alpha}_{s}}{N-1}\right)^{7}+\ldots
$$

Under the above conditions one may calculate $\gamma_{L}\left(N, a_{s}\right)$ in the whole complex plane. It is a bounded function of $\rho=(N-1) / \bar{\alpha}_{s}$, the singularities of which are branch points [370, 371] at

$$
\rho_{1}=4 \log 2, \quad \rho_{2,3}=-1.41048 \pm 1.97212 i .
$$

Its analytic structure is shown in Figure 19. Note, that the resummed form of $\gamma_{L}\left(N, a_{s}\right)$ removes all the fixed-order pole singularities of Eq. (13.14) into branch cuts. Since the known NLO resummed anomalous dimensions are functions of $\gamma_{L}\left(N, a_{s}\right)$ which introduce no further singularities the contour integral around the singularities of the problem has to cover the three BFKL branch points, the singularities of the input distributions along the real axis left of $N=1$, and the remaining singularities of the fixed order anomalous dimensions at the non-positive integers [303, 371. Any finite correction to $\gamma_{L}$ may thus lead to essential changes of the corresponding numerical results. Early numerical studies on the impact of the LO resummed anomalous dimensions were performed in [372] and more recently in Refs. [303, 370, 373].

The next-to-leading order resummed anomalous dimensions are given by

$$
\widehat{\gamma}_{N L}\left(N, \alpha_{s}\right)=-2\left(\begin{array}{ll}
\frac{C_{F}}{C_{A}}\left[\gamma_{q g}^{N L}-\frac{8}{3} a_{s} T_{F}\right] & \gamma_{q g}^{N L} \\
\gamma_{g q}^{N L} & \gamma_{g g}^{N L}
\end{array}\right)
$$

with $T_{F}=T_{R} N_{f}$. The quarkonic contributions were calculated in Ref. [374], as well as the resummed coefficient functions $c_{2}\left(N, a_{s}\right)$ and $c_{L}\left(N, a_{s}\right)$. In [375] $c_{L}\left(N, a_{s}\right)$ has also been calculated, giving a result which differs form the one in [374] starting with $O\left(a_{s}^{4}\right) . \quad \gamma_{g g}^{N L}$ was computed in [376, 377]. In the DIS-scheme $\gamma_{q g}^{N L}$ is found to be an analytic, scale-independent function of $\gamma_{L}\left(N, a_{s}\right)$ and reads

$$
\gamma_{q g}^{N L, D I S}\left(N, \alpha_{s}\right)=T_{F} \frac{\alpha_{s}}{6 \pi} \frac{2+3 \gamma_{L}-3 \gamma_{L}^{2}}{3-2 \gamma_{L}} \frac{\left[B\left(1-\gamma_{l}, 1+\gamma_{L}\right)\right]^{3}}{B\left(2+2 \gamma_{L}, 2-2 \gamma_{L}\right)} R\left(\gamma_{L}\right)
$$

where $R(\gamma)$ is given by

$$
R(\gamma)=\frac{1}{\gamma \sqrt{-\chi_{0}^{\prime}(\gamma)}} \exp \left\{\frac{1}{2} \int_{0}^{\gamma} d z \frac{2 \psi^{\prime}(1)-\psi^{\prime}(1-z)-\psi^{\prime}(z)}{\chi_{0}(z)}+\chi_{0}(z)\right\} .
$$

in [374]. In a re-analysis in 375]

$$
R(\gamma)=\frac{1}{-\gamma^{2} \chi_{0}^{\prime}(\gamma)} \exp \left\{\frac{1}{2} \int_{0}^{\gamma} d z \frac{2 \psi^{\prime}(1)-\psi^{\prime}(1-z)-\psi^{\prime}(z)}{\chi_{0}(z)}+\chi_{0}(z)\right\} .
$$

has been obtained, with a different pre-factor. A future calculation of the 4th order Wilson coefficient for the longitudinal structure function in the $\overline{\mathrm{MS}}$ scheme may clarify this question further. 
The NLO resummed gluon anomalous dimension $\gamma_{g g}^{N L}$ was calculated in the $Q_{0}$-scheme [378]. Here, a scale $Q_{0}^{2} \gg \Lambda_{\mathrm{QCD}}^{2}$ is introduced suppressing $k_{\perp}$ effects for $k^{2}<Q_{0}^{2}$, see also [379].14 One has to solve the Bethe-Salpeter equation

$$
(N-1) G_{N}\left(q_{1}, q_{2}\right)=\delta^{D-2}\left(q_{1}-q_{2}\right)+\int d^{D-2} q_{3} K\left(q_{1}, q_{2}\right) G_{N}\left(q_{3}, q_{2}\right)
$$

with

$$
K\left(q_{1}, q_{2}\right)=\delta^{D-2}\left(q_{1}-q_{2}\right) 2 \omega\left(q_{1}\right)+K_{\text {real }}\left(q_{1}, q_{2}\right)+K_{\text {virtual }}\left(q_{1}, q_{2}\right)
$$

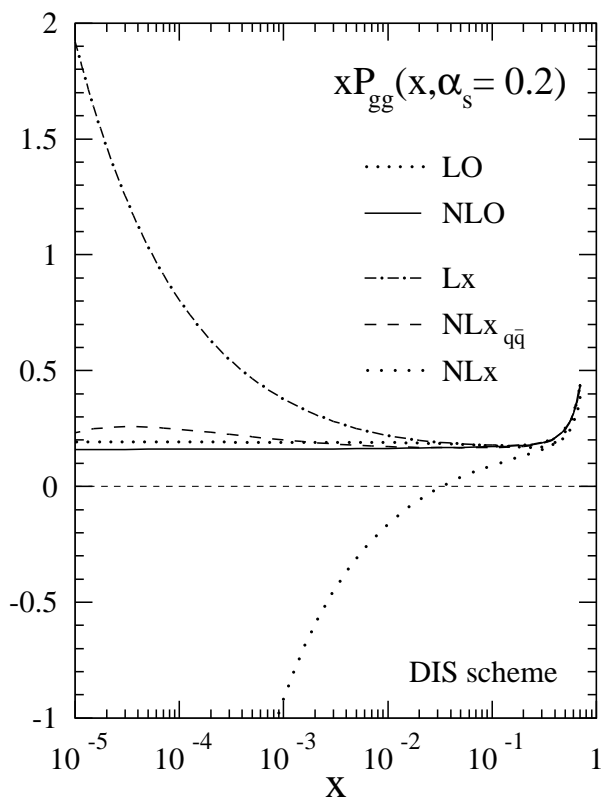

Figure 20: Different contributions to the resummed splitting function $x P_{g g}\left(x, \alpha_{s}\right)$ in the DIS-scheme; Lx: LO BFKL; NLx $q \bar{q}$ : NLO BFKL quarkonic contr.; NLx: NLO BFKL; from [380], (C)(1998) by World Scientific.

For $q_{1}^{2} \gg q_{2}^{2}$ one diagonalizes as in the LO case using formally the same Ansatz:

$$
\int d^{D-2} d q_{2} K\left(q_{1}, q_{2}\right)\left(q_{2}^{2}\right)^{\gamma-1}=\bar{\alpha}_{s}\left[\chi_{0}(\gamma)-\frac{\bar{\alpha}_{s}}{4} \delta\left(\gamma, q_{1}^{2}, \mu^{2}\right)\right]\left(q_{1}^{2}\right)^{\gamma-1} .
$$

Here the scale-invariant LO eigenvalue $\bar{\alpha}_{s} \chi_{0}(\gamma)$ is supplemented by the NLO correction term $\left(\bar{\alpha}_{s}^{2} / 4\right) \delta\left(\gamma, q_{1}^{2}, \mu^{2}\right)$

$$
\begin{aligned}
\delta\left(\gamma, q_{1}^{2}, \mu^{2}\right)= & -\left(\frac{67}{9}-2 \zeta(2)-\frac{10}{27} N_{f}\right) \chi_{0}(\gamma)+4 \Phi(\gamma)-\frac{\pi^{3}}{\sin ^{2}(\pi \gamma)} \\
& +\frac{\pi^{2}}{\sin ^{2}(\pi \gamma)} \frac{\cos (\pi \gamma)}{1-2 \gamma}\left[\left(22-\beta_{0}\right)+\frac{\gamma(1-\gamma)}{(1+2 \gamma)(3-2 \gamma)}\left(1+\frac{N_{f}}{3}\right)\right] \\
& +\frac{\beta_{0}}{3} \chi_{0}(\gamma) \log \left(\frac{q_{1}^{2}}{\mu^{2}}\right)+\left[\frac{\beta_{0}}{6}+\frac{d}{d \gamma}\right]\left[\chi_{0}^{2}(\gamma)+\chi_{0}^{\prime}(\gamma)\right]-6 \zeta_{3},
\end{aligned}
$$

with

$$
\Phi(\gamma)=\int_{0}^{1} \frac{d z}{1+z}\left[z^{\gamma-1}+z^{\gamma}\right]\left[\operatorname{Li}_{2}(1)-\operatorname{Li}_{2} 3(z)\right] .
$$

\footnotetext{
${ }^{14}$ For a transformation into the DIS-scheme cf. 303.
} 


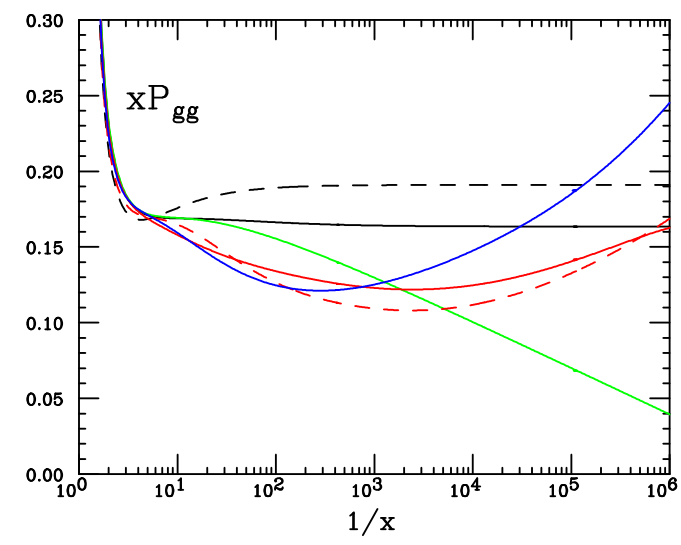

Figure 21: The gluon splitting functions $x P_{g g}$ plotted with $\alpha_{s}=0.2$ and $N_{f}=4$ The curves are (from top to bottom for $x P_{g g}$ at $x \sim 0.2$ ): fixed order perturbation theory LO (black dashed), NLO (black solid), NNLO (green), resummed LO (red dashed) and NLO in $Q_{0} \overline{\mathrm{MS}}$ scheme (red solid) and in the $\overline{\mathrm{MS}}$ scheme (blue); from [381], (C)(2008) by Elsevier Science.

Whereas the terms in the first two lines of Eq. (13.23) do contain contributions to the anomalous dimension up to $O\left(a_{s}^{2}\right)$ the third line contributes only with three-loop order. The agreement with the perturbative results to 2-loop order has been known in the 1990ies. The $O\left(a_{s}^{3}\right)$ from (13.23), given in the DIS-scheme, agree with the perturbative result [60] after transforming it to the $\overline{\mathrm{MS}}$ scheme. The test of even further agreement in higher orders is left for the future. Note that the addend $-6 \zeta_{3}$, being numerically large, contains contributions of the gluonic contribution to the trajectory function $\omega\left(q_{1}^{2}\right)$. The result given in Ref. 382 was confirmed in a different calculation by Ref. [383. A departing value was reported in 384,15

Numerical results on the impact of the leading and next-to-leading anomalous dimensions and coefficient functions were provided in a series of detailed studies, see e.g. [303, 370,380,385] and references therein. The matrix formalism for the solution of the all order evolution equations, extending a first approach in Ref. 304 to all orders, both for hadronic and photon structure functions, is described in Ref. 303] in detail. The quarkonic contributions lead to a strong enhancement of both $F_{2}\left(x, Q^{2}\right)$ and $F_{L}\left(x, Q^{2}\right)$ at small $x$ during the evolution. However, already simple choices for the yet unknown less singular contributions, cf. (13.11), diminish these effects sizably so that a final conclusion cannot be drawn at present. In the case of the resummed gluon anomalous dimension the NLO contributions are found to be extremely large and negative. The large rise due to the LO BFKL term is already canceled to the level of the fixed order contributions by the purely quarkonic contribution to $\gamma_{g g}^{N L}$, see Figure 20. Adding also the gluonic contribution leads to negative values for the resummed splitting function already for $\alpha_{s}=0.2$ and $x \simeq 0.01$ which has to be regarded as unphysical. The LO and NLO resummed contributions to the gluon anomalous dimension seem to represent the first terms of a diverging series, which might be eventually resummed.

An interesting approach in this direction was performed in Refs. [381, 386], and related work in [387,388]. In [381,386] the coupled evolution equations

$$
\frac{d}{d t} f^{+}(N, t)=\gamma^{+}\left(a_{s}(t), N\right) f^{+}(N, t), \quad \frac{d}{d \xi} f^{+}(x, M)=\chi\left(\hat{a_{s}}, M\right) f^{+}(x, M),
$$

with $t=\log \left(Q^{2} / Q_{0}^{2}\right), \xi=\ln (1 / x), f^{+}(x, M)=\int_{-\infty}^{+\infty} d t \exp (-M t) f^{+}(x, t)$, and $N$ the Mellin variable. $\gamma^{+}\left(a_{s}, N\right)$ is the eigenvalue of the singlet-evolution kernel containing poles at $N=1$ and $\chi\left(\hat{a}_{s}, M\right)$

\footnotetext{
${ }^{15}$ Despite the fact that in Ref. [384 the quark contribution to $\omega\left(q_{1}^{2}\right)$ agrees with [382, 383, it may still be that the calculation in [384] addresses a different quantity.
} 
denotes the BFKL-kernel, where $\hat{a}_{s}$ is the operator $a_{s}(t \rightarrow-\partial / \partial M)$. Eqs. (13.25) give rise to the so-called duality relation

$$
\chi\left(a_{s}, \gamma^{+}\left(a_{s}, N\right)\right)=N, \quad \gamma^{+}\left(a_{s}, \chi\left(a_{s}, M\right)\right)=M
$$

in the small- $x$ region. These relations are used to organize the resummation of the small- $x$ contributions, which leads to a gradual stabilization of the dominant splitting functions at low $x$. For the splitting function $x P_{g g}(x)$ this is illustrated in Figure 21, including all the presently known information. In the $\overline{\mathrm{MS}}$ scheme significantly larger values are found than in the 3-loop fixed order calculation at $x \sim 10^{-4}$ and below.

Finally, we would like to mention that the effect of potential sub-leading contributions to the LO resummed anomalous dimension may be studied within $\Phi^{3}$ theory in $D=6$ dimensions, as a simple model with 3-boson interactions at the perturbative level. Here the leading order resummed anomalous dimension can be calculated for all values of $x$ solving a Bethe-Salpeter equation [389]. The corresponding singularity at fixed orders is located at $N=-1$ due to the scalar field. In [390] the LO small- $x$ resummed terms were compared to the complete ladder solution adding the NLO corrections. In this case it turns out that the pure small- $x$ resummed terms do not give the correct result. To further consolidate the knowledge on parton distributions in the small- $x$ region it seems to be necessary to calculate at least one further resummed series, i.e. NNLO BFKL.

\section{Resummation at Large Values of $x$}

The dominant contributions to the splitting functions and Wilson coefficients in the large- $x$ region result form the terms

$$
\left[\frac{\ln ^{k}(1-x)}{1-x}\right]_{+}, \quad \delta(1-x), \quad \ln ^{l}(1-x), \quad k, l \in \mathbb{N}, k \geq 0, l>0 .
$$

This is best seen in Mellin space, where

$$
\begin{aligned}
\frac{1}{k !} \mathbf{M}\left[\left(\frac{\ln ^{k}(1-x)}{1-x}\right)_{+}\right](N) & =(-1)^{k+1} S_{\underbrace{S_{1} \ldots, 1}_{k+1}}(N-1) \\
\mathbf{M}[\delta(1-x)](N) & =1 \\
\frac{1}{k !} \mathbf{M}\left[\ln ^{k}(1-x)\right](N) & =(-1)^{k} \frac{1}{N} \underbrace{S_{1, \ldots, 1}}_{k}(N) .
\end{aligned}
$$

The harmonic sums with equal index form a polynomial in single harmonic sums, cf. [174, 223],

$$
S_{\underbrace{1, \ldots, 1}_{k}}(N)=\frac{1}{k} \sum_{l=1}^{k} S_{\underbrace{1, \ldots, 1}_{k-l}}(N) S_{l}(N) .
$$

In the limit $|N| \rightarrow \infty$ one obtains

$$
S_{\underbrace{1, \ldots, 1}}(N) \propto \frac{1}{k !} S_{1}^{k}(N)+O\left(S_{1}^{k-2}(N) S_{2}(N)\right)
$$

and

$$
S_{1}(N) \propto \ln (\bar{N})+\frac{1}{2 N}+\frac{1}{12 N^{2}}-\frac{1}{120 N^{4}}+O\left(\frac{1}{N^{6}}\right)
$$


with $\bar{N}=N+\gamma_{E}$.

Let us consider the splitting functions $P_{i k}$ and Wilson coefficients $C_{p, i}$

$$
\begin{aligned}
P_{i k}\left(x, a_{s}\right) & =\sum_{j=0}^{\infty} a_{s}^{j+1} P_{i k}^{(j)}(x) \\
C_{p, i}\left(x, a_{s}\right) & =\delta_{p, 2(3)} \delta(1-x)+\sum_{j=1}^{\infty} a_{s}^{j} c_{p, i}^{(j)}(x) .
\end{aligned}
$$

The large $x$ structure for the spitting functions $P_{i k}$ and Wilson coefficients known up to $O\left(\alpha_{s}^{3}\right)$ [59 61] is

$$
\begin{aligned}
P_{k k}^{(l)}(x) & =A_{l+1}(1-x)_{+}+B_{l+1} \delta(1-x)+C_{l+1} \ln (1-x)+O\left[(1-x)^{k \geq 1} \ln ^{l+1}(1-x)\right] \\
P_{i \neq k}^{(l)}(x) & =\sum_{j=0}^{2 l-1} D_{k l}^{(l, j)} \ln ^{2 l-j}(1-x)+O(1) \\
c_{a, l}(x) & =\frac{\left(2 C_{F}\right)^{l}}{(l-1) !} p_{q q}(x) \ln ^{2 l-1}(1-x)+O\left[(1-x)^{k \geq-1} \ln ^{2 l-2}(1-x)\right],
\end{aligned}
$$

cf. [391,392]. Here $c_{a, l}(x)$ denotes the non-singlet Wilson coefficient with $p_{q q}(x)=2 /(1-x)_{+}-(1+x)$. The singlet Wilson coefficients are of a similar structure. The splitting function $P_{q q}^{s}(x)$ related to the color factor $d_{a b c} d^{a b c}(7.5)(7.6)$ behaves like $(1-x)^{k \geq 1} \ln (1-x)$ for large values of $x$, [391].

In Mellin-space the resummed Wilson coefficients have the structure [393, 394]

$$
C(N)=g_{0}\left(a_{s}\right) \exp \left\{\ln (N) g_{1}(\lambda)+g_{2}(\lambda)+a_{s} g_{3}(\lambda)+O\left(a_{s}^{2} f(\lambda)\right)\right\},
$$

with $g_{0}\left(a_{s}\right)$ the normalization and $\lambda=a_{s} \beta_{0} \ln (N)$. In the flavor non-singlet case the functions $g_{1,2}\left(a_{s}\right)$ are given by

$$
\begin{aligned}
& g_{1}\left(a_{s}\right)=\frac{A_{1}}{\beta_{0} \lambda}[\lambda+(1-\lambda) \ln (1-\lambda)] \\
& g_{2}\left(a_{s}\right)=-\frac{\gamma_{E} A_{1}-B_{1}}{\beta_{0}} \ln (1-\lambda)-\frac{A_{2}}{\beta_{0}^{2}}[\lambda+\ln (1-\lambda)]+\frac{A_{1} \beta_{1}}{\beta_{0}^{3}}\left[\lambda \ln (1-\lambda)+\frac{1}{2} \ln ^{2}(1-\lambda)\right],
\end{aligned}
$$

with

$$
A_{1}=4 C_{F}, \quad A_{2}=8 C_{F}\left[\left(\frac{67}{18}-\zeta_{2}\right) C_{A}-\frac{5}{9} N_{f}\right], \text { 393] } B_{1}=-3 C_{F} \text { [394. }
$$

The universal part of $g_{3} \propto a_{s} \ln (N)$ was derived in [395].

The leading order resummation corrections beyond $O\left(a_{s}^{3}\right)$ valid for large values of $N$ in the non-singlet case have been calculated in [396]. Next-to-leading-log contributions were accounted for in [391, 397].

One may investigate the large- $x$ structure of the 4-loop anomalous dimensions studying schemeinvariant evolution equations, cf. [152-155], for the $F_{2}, F_{\phi}$-system, with $\phi$ a scalar particle coupling to the gluon as the photon couples to the quarks. The specific structure of the scheme-invariant kernels to the level of $a_{s}^{3}$ is assumed to hold at $a_{s}^{4}$. Knowing the 3-loop Wilson coefficients, one may determine under this assumption the large $x$ behaviour of the singlet splitting functions $P_{i j}^{(3)}(x)$ in the highest two powers in $\ln (1-x)$, cf. [398]. A resummation of the contributions to $P_{q g}, P_{g q}, C_{2, g}$ and $C_{L, g}$ has been performed in [392]. A systematic approach to obtain even higher order terms in case of the large- $x$ resummation has been proposed in Ref. [399]. 


\section{Sum Rules and Integral Relations}

Deep-inelastic structure functions obey a series of sum rules for special moments or even integral relations, which are of interest for experimental tests. These relations are of different rigor. In most of the cases they receive radiative corrections, mass corrections, and in some cases even non-perturbative corrections a priori. Using current algebra techniques many sum rules have been investigated in [400]. A larger series of sum rules having been proposed for polarized scattering have been analyzed in [94]. Quark mass and QCD corrections have been given in [288, 400]. In the following we give a brief description of the main sum rules and comment on the status of available QCD corrections.

Adler sum rule [401:

This sum rule is rigorous and neither obtains QCD nor mass corrections.

$$
\int_{0}^{1} \frac{d x}{x}\left[F_{2}^{\bar{\nu} p}\left(x, Q^{2}\right)-F_{2}^{\nu p}\left(x, Q^{2}\right)\right]=K\left(N_{f}\right),
$$

with $K(3)=2+2 \sin ^{2} \theta_{c},\left(S U_{F}(3)\right)$ and $K(4)=2,\left(S U_{F}(4)\right)$, and $\theta_{c}$ the Cabibbo angle, cf [288, 400]. Unpolarized Bjorken sum rule 402]:

The sum rule refers to the charged current structure functions $F_{1}$

$$
\int_{0}^{1} d x\left[F_{1}^{\bar{\nu} p}\left(x, Q^{2}\right)-F_{1}^{\nu p}\left(x, Q^{2}\right)\right]=K\left(N_{f}\right) A^{\mathrm{F}_{1}}\left(N_{f}, Q^{2}\right),
$$

with $K(3(4))=1+\sin ^{2} \theta_{c}$, (1) and $A^{\mathrm{F}}\left(N_{f}, Q^{2}\right)=1+O\left(a_{s}\right)$. The 3-loop QCD corrections to $A^{F_{1}}$ were given in [403].

Gross-Llewellyn Smith sum rule [404]:

Likewise, the combination for the charged current structure functions $F_{3}$ yields

$$
\int_{0}^{1} d x\left[F_{3}^{\bar{\nu} p}\left(x, Q^{2}\right)+F_{3}^{\nu p}\left(x, Q^{2}\right)\right]=K\left(N_{f}\right) A^{\mathrm{F}_{3}}\left(N_{f}, Q^{2}\right),
$$

with $K(3(4))=6-2 \sin ^{2} \theta_{c}, \quad(6)$. The 3-loop QCD corrections to $A^{\mathrm{F}_{3}}\left(N_{f}, Q^{2}\right)$ were given in [405] and the 4-loop corrections in [406].

Polarized Bjorken sum rule [407]:

The sum rule refers to the flavor non-singlet combination

$$
\int_{0}^{1} d x\left[g_{1}^{e p}\left(x, Q^{2}\right)-g_{1}^{e n}\left(x, Q^{2}\right)\right]=\frac{1}{6}\left|\frac{g_{A}}{g_{V}}\right| A^{\mathrm{g}_{1}}\left(N_{f}, Q^{2}\right),
$$

with $g_{A, V}$ the neutron decay constants, $g_{A} / g_{V} \approx-1.26$. The 3 -loop QCD corrections to $A^{\mathrm{g}_{1}}\left(N_{f}, Q^{2}\right)$ were given in [405] and the 4-loop corrections in [408].

Gerasimov-Drell-Hearn sum rule [409, 410]:

This sum rule is given by the first moment of the polarized structure function $g_{1}^{p, n}\left(x, Q^{2}\right)$ in the form, cf. e.g. [338, 411, 412],

$$
I_{p, n}\left(Q^{2}\right)=2 \frac{M^{2}}{Q^{2}} \int_{0}^{x_{0}} d x g_{1}^{p, n}\left(x, Q^{2}\right)= \begin{cases}-\frac{1}{4} \mu_{p, n}^{2}, & Q^{2} \rightarrow 0 \\ \frac{2 M^{2}}{Q^{2}} \Gamma_{1}^{p, n}, & Q^{2} \rightarrow \infty\end{cases}
$$

at proton and neutron targets, with $x_{0}=Q^{2} /\left(2 M m_{\pi}+m_{\pi}^{2}+Q^{2}\right), \mu_{p, n}$ the anomalous magnetic moment of the proton or nucleon (1.1), and $\Gamma_{1}$ the first moment of the structure function $g_{1}$ at infinite space-like momentum transfer. The sum-rule has a very strong $Q^{2}$-evolution for low values of the virtuality. In case of proton targets it changes sign between $Q^{2}=0$ and $Q^{2} \approx 1 \mathrm{GeV}^{2},[338$. 


$$
\int_{0}^{1} d x g_{2}\left(x, Q^{2}\right)=0
$$

In the derivation of the sum rule it was assumed that the large $\nu$-behaviour of the associated amplitude $A_{2}$, cf. [94], is governed by Regge-theory and it behaves as $A_{2}\left(Q^{2}, \nu\right) \sim \nu^{-1-\epsilon}, \epsilon<0$. However, this may be questioned, see [414. It was argued in [415] that Regge cuts spoil the sum rule, which would vanish for large $Q^{2}$, however. The sum-rule may be invalidated in case of a short-distance singularity [414], see also [16]. The Burkhardt-Cottingham sum rule cannot be expressed in terms of expectation values of (axial-)vector current operators and not be derived using the light-cone expansion, see e.g. [94], although a formal analytic continuation for general values of the Mellin variable $N$ would suggest it. It is wellknown, however, that specific moments may have a particular behaviour. The $O\left(\alpha_{s}\right)$ corrections [400], target mass corrections [93] and massive quark corrections [417] do not alter this relation. A brief review on this sum rule has been given in Ref. [414.

\section{Efremov-Teryaev-Leader sum rule 418:}

This sum rule refers to the valence $(\mathrm{V})$ contributions of the polarized structure functions $g_{1,2}$ related to the twist-3 operator matrix element $d_{1}^{\gamma q, V}$

$$
\int_{0}^{1} d x x\left[g_{1}^{\gamma q, \mathrm{~V}}\left(x, Q^{2}\right)+2 g_{1}^{\gamma q, \mathrm{~V}}\left(x, Q^{2}\right)\right]=\frac{e_{q}^{2}}{8} d_{1}^{\gamma q, V}=0 .
$$

It receives quark mass corrections and is thus only valid in the limit $m_{q} \rightarrow 0$, cf. [94]. It holds under certain conditions also in the presence of target mass corrections [93].

Ellis- Jaffe sum rule [419]:

This sum rule is given by the integral

$$
\int_{0}^{1} d x g_{1}^{p(n)}\left(x, Q^{2}\right)=C^{\mathrm{NS}}\left(Q^{2}\right) A^{\mathrm{NS}}\left(Q^{2}\right)+C^{\mathrm{S}}\left(Q^{2}\right) A^{\mathrm{S}}\left(Q^{2}\right)
$$

It was originally devised to describe the nucleon spin composition. However, the nucleon spin receives also gluonic and angular momentum contributions. The latter can in principle be measured using nonforward scattering [80]. Here $C^{\mathrm{NS}, \mathrm{S}}$ denote Wilson coefficients and $A^{\mathrm{NS}, \mathrm{S}}$ the corresponding OMEs in the flavor non-singlet and singlet case. The non-singlet current is conserved for massless quarks, while the singlet current is not conserved, which causes a non-vanishing singlet anomalous dimension at $O\left(a_{s}^{2}\right)$. The 3- and 4-loop QCD corrections to this sum rule have been calculated in [420,421].

Gottfried sum rule [422]:

$$
\left.\int_{0}^{1} \frac{d x}{x}\left[F_{2}^{\gamma p}\left(x, Q^{2}\right)-F_{2}^{\gamma n}\left(x, Q^{2}\right)\right]=\frac{1}{3} \int_{0}^{1} d x\left[u_{v}\left(x, Q^{2}\right)-d_{v}\left(x, Q^{2}\right)-\left(\bar{d}\left(x, Q^{2}\right)-\bar{u}\left(x, Q^{2}\right)\right)\right)\right] .
$$

This sum rule is non-rigorous since the distributions $\bar{d}\left(x, Q^{2}\right)$ and $\bar{u}\left(x, Q^{2}\right)$ are found to be different and are non-perturbative quantities. It is currently studied in lattice simulations, cf. Figure 16. The 3-loop corrections to the sum-rule follow from the Wilson coefficients [61]. The large- $N_{c}$ limit was studied in [423].

Integral Relations at Twist 2 and 3 [93, 266]:

The twist-2 and twist-3 contributions to the structure functions $g_{1,2}\left(x, Q^{2}\right)$ obey the relations

$$
\begin{aligned}
& g_{2}^{\tau=2}\left(x, Q^{2}\right)=-g_{1}^{\tau=2}\left(x, Q^{2}\right)+\int_{x}^{1} \frac{d y}{y} g_{2}^{\tau=2}\left(y, Q^{2}\right) \\
& g_{1}^{\tau=3}\left(x, Q^{2}\right)=\frac{4 M^{2}}{Q^{2}}\left[g_{2}^{\tau=3}\left(x, Q^{2}\right)-2 \int_{x}^{1} \frac{d y}{y} g_{2}^{\tau=3}\left(y, Q^{2}\right)\right] .
\end{aligned}
$$


The Wandzura-Wilczek relation (15.10) [266], originally derived in the massless case, remains valid in case of quark- and target mass corrections [93], for the gluonic contributions [265], for non-forward scattering [301,424], and diffractive scattering [302,425]. It can be derived using the covariant parton model, as well as relations for other polarized structure functions containing twist-3 contributions, cf. [145, 150]. In the limit of vanishing target masses $g_{1}$ does not receive a twist-3 contribution. Eqs. (15.10,15.11) allow to disentangle the fermionic twist- 2 and 3 contributions to $g_{1}$ and $g_{2}$.

\section{Higher Twist Contributions}

Beyond the leading twist contributions, i.e. twist $\tau=2$ in Quantum Chromodynamics, a quite different picture emerges for the structure functions both in the polarized and unpolarized case. With the twist-3 contributions to polarized deep-inelastic scattering the problem of the operator mixing becomes more involved since the number of operators contributing at total spin $N$ is growing with $N$. Both the anomalous dimensions and Wilson coefficients have been calculated during the last three decades at the level of the $O\left(a_{s}\right)$ corrections. The twist-3 anomalous dimensions have been derived in Refs. [426, 427]. The evolution in the non-singlet case in the large $N_{c}$ limit has been studied in [428] and terms beyond this limit were derived in [429]. The twist-3 non-singlet and singlet anomalous dimensions have been calculated using light-ray operators in [430,431]. The leading order Wilson coefficients were computed in [432] in the non-singlet and in [433] in the singlet case. The renormalization of gauge invariant operators contributing to the structure function $g_{2}\left(x, Q^{2}\right)$ has been studied in [434]. In Ref. [435] the gluonic contributions to the structure function $g_{2}\left(x, Q^{2}\right)$ were calculated. The complete non-singlet and singlet evolution of the twist-3 moments was derived in [436].

The data on $g_{2}\left(x, Q^{2}\right)$ [122, 135] have still large errors, which can be substantially improved at a facility like the EIC [137, 138]. For first estimates on the behaviour of the twist-3 part of $g_{2}\left(x, Q^{2}\right)$ see e.g. in [437]. Yet the experimental errors on the twist-3 contribution of the structure function $g_{2}\left(x, Q^{2}\right)$ are large [295]. First lattice measurements of the lowest twist-3 moment have been performed in [438, 439] at larger pion masses and have to be refined for realistic values of $m_{\pi}$ in the future.

In the unpolarized case the contributions of higher (dynamical) twist are of $\tau=4,6, \ldots$ They are largely suppressed in the limit of large virtualities $Q^{2}$. However, the experimental data often exhibit a correlation between $x$ and $Q^{2}$ due to similar values of $S$. Furthermore, in neutral current deep-inelastic scattering the largest statistics is located in the region of lower values of $Q^{2}$. It is difficult to decide from which scale $Q^{2}$ onwards a data sample is widely free of higher twist contributions, for which sometimes a cut of $W^{2}=12.5-15 \mathrm{GeV}^{2}$ is proposed [199,440]. This cut has been verified in the non-singlet analysis [199].

The local operators of higher twist can be constructed systematically near the light-cone. They are formed by more external quark and gluon fields than the twist- 2 operators and potential contributions of lower twist operators in case mass scales are present. A twist-4 operator is given e.g. by

$$
: \bar{\psi}(x) \gamma_{\mu_{1}} \partial_{\mu_{2}} \ldots \partial_{\mu_{m}} \psi(x) \bar{\psi}(y) \gamma_{\nu_{1}} \partial_{\nu_{2}} \ldots \partial_{\nu_{n}} \psi(y):
$$

A systematic twist-decomposition has to be performed, cf. [140]. One forms operator matrix elements with these operators between nucleon states. A 'partonic' interpretation assumes, that all external lines can be factorized. The corresponding contributions to deep-inelastic structure functions are then similar as in the case of twist-2, with the generalization that both the Wilson coefficients and OMEs depend on $n-1$ dimensionless parameters $\sum_{i=1}^{n-1} x_{i}=x$, where $n$ denotes the number of external fields. These parameters, except $x$, cannot be measured in the deep-inelastic process. 

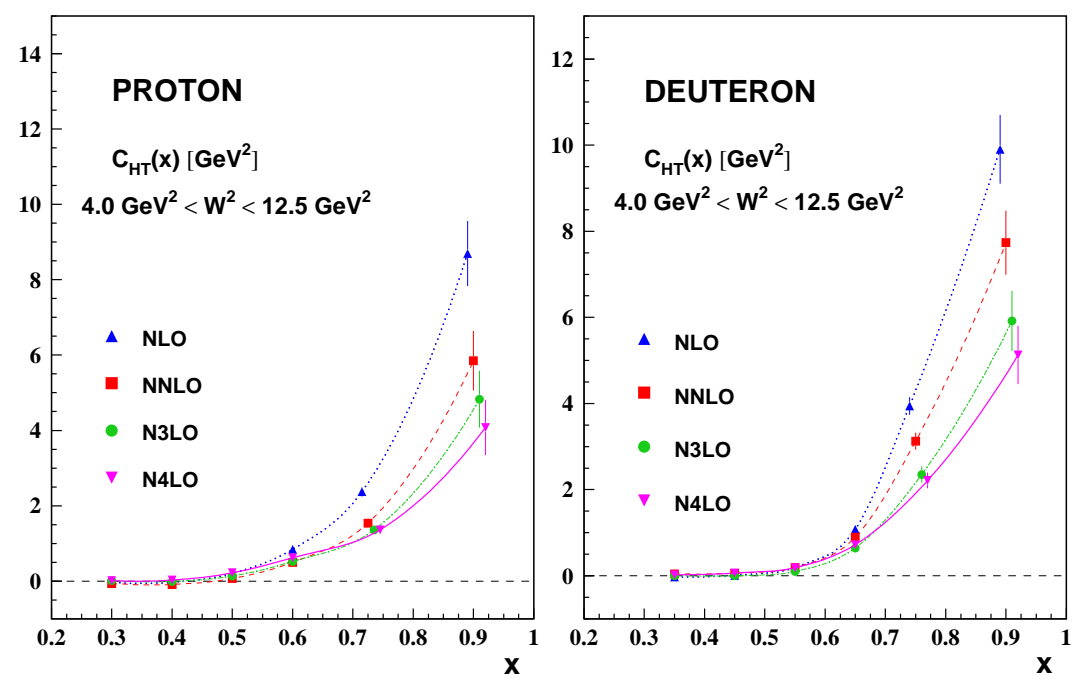

Figure 22: Comparison of the higher twist coefficient $C_{H T}(x) \equiv C_{2}^{\tau=4}(x)$ in the large $\mathrm{x}$ region for the proton data as function of $x$ in a NLO (dotted lines), NNLO (dashed lines), N3LO analysis (dash-dotted lines) for the non-singlet QCD Wilson coefficient (full lines). Some bin centers are slightly shifted for better visibility; from [455], ()(2010) by Elsevier Science.

Early theoretical investigations of the structure of higher twist operators and their anomalous dimensions in $D=6 \Phi^{3}$-theory [441] and QCD [186, 426, 442, 448] revealed the principal structure of these contributions. The lowest order anomalous dimensions have been calculated in [186, 445, 448] and the Wilson coefficients, in different operator bases, in [443,444,446]. More recently also gluonic operators were considered [447]. A systematic study of higher twist light-cone distribution amplitudes was given in [448. The renormalization of these operators has been worked out systematically in [449]. The evolution of the lowest twist-4 moments at leading order has been studied in [450] recently.

Due to the fact that the OMEs of higher twist operators cannot be measured experimentally one may envisage their determination with lattice simulations. At present, investigations of this kind are still at the beginning. In Mellin-space the basis of higher twist operators grows considerably with the value of the Mellin variable $N$ and an according number of OMEs has to be measured on the lattice, which further complicates quantifications of higher twist effects ab initio.

Estimates of higher twist effects have been obtained studying renormalon corrections to sum-rules and deep-inelastic structure functions, see [451] and for reviews Refs. [452,453.

Because of the complications mentioned the extraction of higher twist contributions to deep-inelastic structure functions is usually being performed applying a suitable Ansatz for these contributions:

$$
F_{i}\left(x, Q^{2}\right)=F_{i}^{\tau=2}\left(x, Q^{2}\right)\left[1+\frac{C_{i}^{\tau=4}\left(x, Q^{2}\right)}{Q^{2}}+\frac{C_{i}^{\tau=6}\left(x, Q^{4}\right)}{Q^{2}}+\ldots\right] .
$$

Sometimes one considers the higher twist terms in (16.2) in additive form. In many cases these contributions are fitted together with the PDFs, cf. [454,455]. Since the logarithmic scaling violations of the higher twist coefficients are in general different from those of the lowest twist it was suggested to proceed in a different way, cf. [199,455]. In the flavor non-singlet case the lowest twist contributions to the structure functions may be determined in the region which is free of higher twist terms. One then extrapolates the twist-2 distributions into the region, where higher twist contributions are present and measures the difference. The size of higher twist contributions thus obtained stabilizes including higher order QCD corrections to the leading twist terms. The inclusion of the leading twist 3-loop Wilson coefficients yields the dominant contribution and effectively describes the $\mathrm{N}^{3} \mathrm{LO}$ terms, while the $\mathrm{N}^{4} \mathrm{LO}$ term is approximate only as it refers to the soft exponentiations [396]. In Figure 22 the corresponding results are shown for a world analysis in the valence region for proton and deuteron data. 

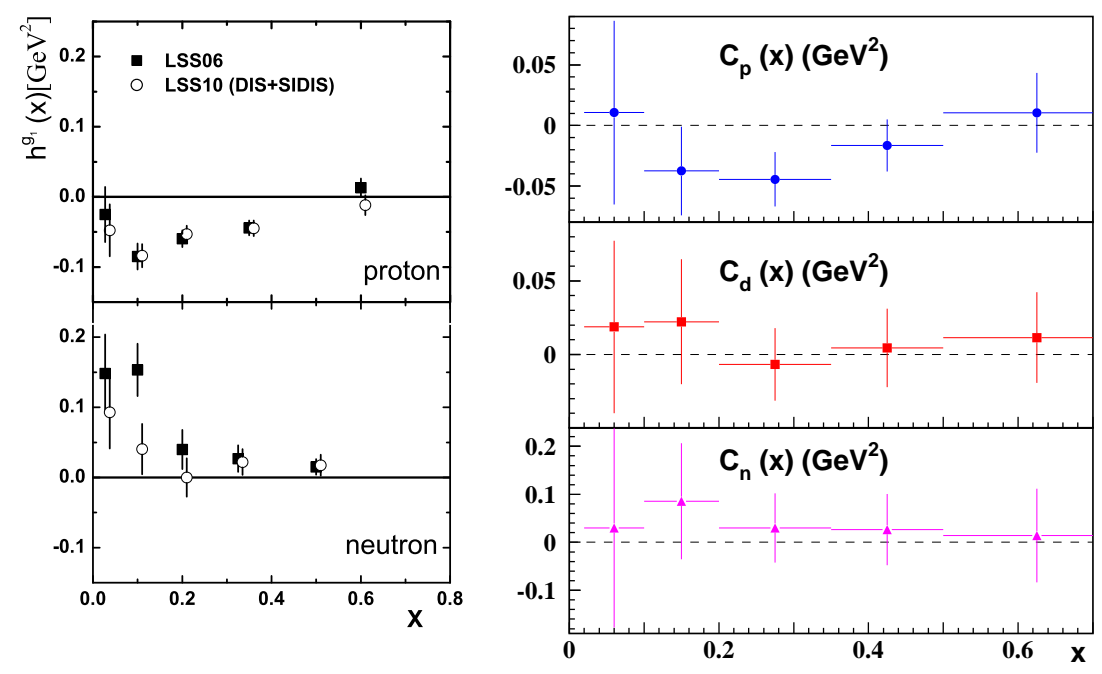

Figure 23: Left: comparison between the higher twist values corresponding to the fits of inclusive DIS LSS06 [456] and combined inclusive and SIDIS data set LSS10 [342] (2010) by the American Physical Society. Right: The additive higher twist coefficients $C_{p}(x), C_{d}(x)$ and $C_{n}(x)$ [329], () (2010) by Elsevier Science.

A similar analysis is not possible in the polarized case at present, because of the smaller kinematic range in $Q^{2}$ and the larger errors of the data for the structure function $g_{1}\left(x, Q^{2}\right)$. Here the higher twist contributions are fitted as additive terms in an NLO analysis for individual bins, cf. e.g. [329, 342, 456. In Refs. [342, 456] smaller errors have been obtained with an excess at lower values of $x$ in case of neutron targets and proton targets, see Figure 23. In [329] higher twist contributions which are widely compatible with zero have been obtained both for the proton and neutron targets, with a 1-1.5 $\sigma$ effect in two bins for the proton data. To reveal twist-4 contributions to the structure function $g_{1}\left(x, Q^{2}\right)$ more precise data from future experiments are needed.

\section{Nuclear Parton Distribution Functions}

The parton densities for free nucleons and nuclei are different. This has been first demonstrated in detail by the EMC-experiment [457]. One well-known effect is due to the Fermi-motion of nucleons within nuclei [458]. Beyond these contributions other deviations have been observed, in the region of lower values of $x$. Various phenomenological explanations have been proposed, like binding models of nucleons, admixture models, change of nucleon mass, pion enhancement, multi-quark clusters, dynamical rescaling (change of confinement size), shadowing, and others; for reviews see [459]. Nuclear effects also modify the Drell-Yan process, $W^{ \pm} / Z$-production, high $p_{\perp}$ hadron production, $J / \psi$ - and direct photon production.

Recent global data analyses to extract the nuclear PDFs have been carried out by different groups [460 465] extracting nuclear correction factors $R_{i}^{A}\left(x_{N}, Q^{2}\right)$ for the parton densities $f_{i}\left(x, Q^{2}\right)$ in free nucleons. In general the Bjorken variable $x_{N}$ for nuclear PDFs has support $x_{N} \in[0, A]$. However, measured distributions for values of $x_{N}>1$ do rapidly fall. Therefore, one often parameterizes the nuclear PDFs only for the range $x_{N} \in[0,1]$. A recent parameterization was given in Ref. [465. The correction factors $R_{i}^{A}\left(x_{N}, Q^{2}\right)$ are illustrated in Figure 24 for a series of nuclei for the quark distributions $u_{v}, \bar{u}, s$ and the gluon distribution $g$. The $d_{v}$ distribution is assigned the same correction factor as the $u_{v}$ distribution.

The nuclear corrections at very small and very large $x_{N}$ become stronger with the rising value of A. At large $x_{N}$ Fermi-motion effects dominate. Anti-shadowing effects are observed in the region $x_{N} \in[0.01,0.1]$ and the correction factors become smaller than one for even smaller values of $x_{N}$. The comparison of different fits shows that there are still quite some uncertainties. On the theoretical side 
a complete understanding of the factors $R_{i}^{A}$ is not yet obtained, despite a large number of proposed models.

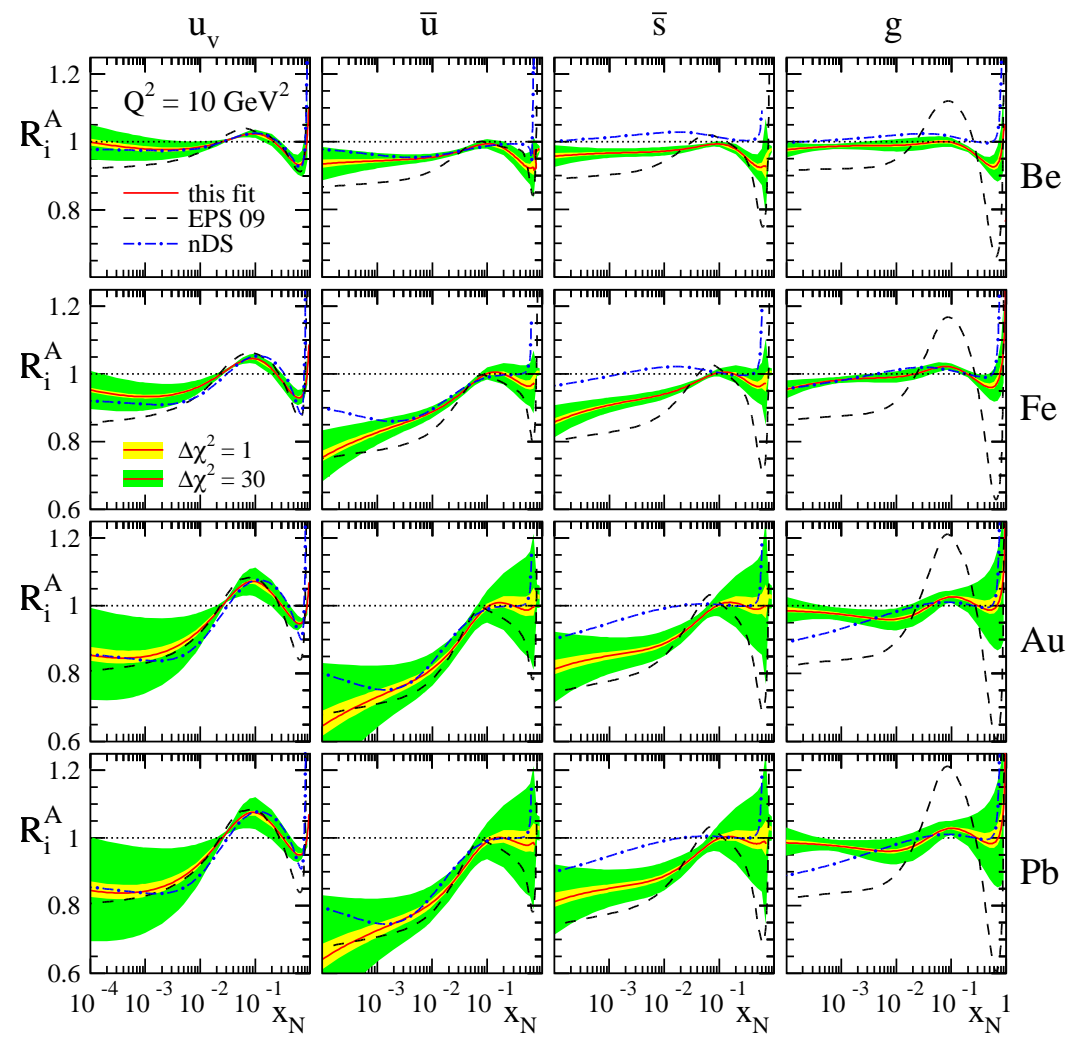

Figure 24: The NLO nuclear modification factors $R_{i}^{A}$ for different parton densities at $Q^{2}=10 \mathrm{GeV}^{2}$ for $\mathrm{Be}, \mathrm{Fe}, \mathrm{Au}$ and $\mathrm{Pb}$ targets as a function of $x_{N}$. The inner (outer) error bands correspond to $\Delta \chi^{2}=1(30)$, respectively. Red lines: [465]; dash-dotted lines [464]; dashed lines [461]; from [465] ()(2012) by the American Physical Society.

Deep-inelastic data analyses include deuteron data to obtain an improved representation of the downquark distributions due to scattering off the neutron. The deuteron data require nuclear corrections [466], which mainly concern Fermi-motion and the off-shell effect, cf. [117]. For the off-shell corrections the Bonn [467] and Paris [468] potentials are used, including the induced differences into the systematic errors. One assumes that the nuclear model suggested in [462] can be applied to the case of light nuclei, like deuterium, which has recently been confirmed for ${ }^{3} \mathrm{He}$ and ${ }^{9}$ Be targets [463. Di-muon production data, which are important to measure the strange-quark distributions, are currently measured at nuclear targets, for which the corrections [469] were applied in [117]. Drell-Yan data of nuclear targets which are used in present PDF-fits are measured in a region with lower nuclear effects, cf. [470].

\section{Conclusions}

During the last 40 years the understanding of the sub-structure of nucleons took a vast development. The theory of strong interactions, QCD, can now be probed at the per-cent level in an increasing number of reactions. High-luminosity measurements performed at SLAC, CERN, DESY, FERMILAB, and JLAB more and more revealed the dynamics of quarks and gluons at short distances. With the advent of the LHC this process will continue in an intimate partnership between precision measurements and precision calculations. During the coming years the data analysis of the HERA-experiments will be finalized. Here a remaining issue is the theoretical understanding of the $e p \rightarrow$ jet cross sections at 
NNLO. Jet physics, the production of $W^{ \pm}$and $Z$-bosons, the Drell-Yan cross section, and inclusive $t \bar{t}$ - as well as single-top production will be important scattering cross sections measured at the LHC to limit the present experimental errors on $\alpha_{s}\left(M_{Z}^{2}\right)$ and to further refine the knowledge on the parton distributions. The precision of the data requests NNLO QCD corrections.

In the more distant future high luminosity facilities like EIC may allow to answer many open questions related to lower energies, like the partonic structure of polarized nucleons, twist-3 effects, and higher twist effects in general, and unravel transverse degrees of freedom, including associated spin effects in detail. This machine will also have the potential to measure $\alpha_{s}\left(M_{Z}^{2}\right)$ very precisely. Proposed facilities like LHeC, on the other hand, allow to further progress into the small $x$ and high $Q^{2}$ domain by ep scattering. Many proposed theoretical concepts can be tested in this way at high precision, giving a boost to even more refined theoretical calculations than carried out at present. Probing ever shorter distances may finally answer the more fundamental question for a possible substructures of quarks, which will request precision, both at the experimental and theoretical side.

Acknowledgment. For discussions I would like to thank J. Ablinger, S. Alekhin, W. Beenakker, Chr. Berger, I. Bierenbaum, J.D. Bjorken, H. Böttcher, S. Catani, V. Drach, A. De Freitas, H. Fritzsch, J. Gracey, A. Hasselhuhn, P. Jimenez-Delgado, M. Klein, S. Klein, S. Moch, A. Petermann, H. Quinn, V. Ravindran, E. Reya, T. Riemann, K. Sasaki, J. Smith, C. Schneider, P. Söding, T. Uematsu, W.L. van Neerven, M. Veltman, J. Vermaseren, A. Vogt, and F. Wißbrock. This work has been supported in part by DFG Sonderforschungsbereich Transregio 9, Computergestützte Theoretische Teilchenphysik, and EU Network LHCPHENOnet PITN-GA-2010-264564. Figure 4 has been drawn using Axodraw [471].

\section{References}

[1] J. Dalton, A New System of Chemical Philosophy, (A. Bickerstaff, Strand, London, 1808).

[2] H. Becquerel, Comptes Rendus 122 (1896) 420; 501; 559; 689; 762.

[3] E. Rutherford, Phil. Mag. Ser. 621 (1911) 669.

[4] J. Chadwick, Nature 129 (1932) 312; Proc. Royal Soc. Lond. Ser. A136 (1932) 692.

[5] H. Yukawa, Proc. Phys.-Math. Soc. Japan 17 (1935) 48; 19 (1937) 712.

[6] R. Frisch and O. Stern, Z. Phys. 85 (1933) 4.

[7] R. Bacher, Phys. Rev. 43 (1933) 1001;

L. Alvarez and F. Bloch, Phys. Rev. 57 (1940) 111.

[8] K. Nakamura et al., [Particle Data Group Collaboration], J. Phys. G 37 (2010) 075021.

[9] R. Hofstadter, Electron scattering and nuclear and nucleon structure. A collection of reprints with an introduction, (New York, Benjamin, 1963), 690 p. and references therein.

[10] D. N. Olson, H. F. Schopper and R. R. Wilson, Phys. Rev. Lett. 6 (1961) 286.

[11] M. Gell-Mann, Phys. Lett. 8 (1964) 214.

[12] G. Zweig, CERN-TH-401, CERN-TH-412, (1964).

[13] W. K. H. Panofsky, Proc. 14th International Conference on High-Energy Physics, Vienna, 1968, J. Prentki and J. Steinberger, eds., (CERN, Geneva, 1968), pp. 23.

[14] R. E. Taylor, Proc. 4th International Symposium on Electron and Photon Interactions at High Energies, Liverpool, 1969, (Daresbury Laboratory, 1969), eds. D.W. Braben and R.E. Rand, pp. 251. 
[15] E. D. Bloom et al., Phys. Rev. Lett. 23 (1969) 930.

[16] M. Breidenbach et al., Phys. Rev. Lett. 23 (1969) 935.

[17] R. E. Taylor, Rev. Mod. Phys. 63, 573 (1991).

[18] H. W. Kendall, Rev. Mod. Phys. 63, 597 (1991).

[19] J. I. Friedman, Rev. Mod. Phys. 63, 615 (1991).

[20] C. G. Callan, Jr. and D. J. Gross, Phys. Rev. Lett. 22 (1969) 156.

[21] J. D. Bjorken, Phys. Rev. 179 (1969) 1547.

[22] R. P. Feynman, In: Proceedings of 3rd International Conference on High Energy Collisions, Stony Brook, N.Y., 5-6 Sep 1969, pp 237.; Phys. Rev. Lett. 23 (1969) 1415.

[23] R. P. Feynman, Photon-hadron interactions, (Benjamin, Reading, MA, 1972), 282 p.

[24] J. D. Bjorken, Proc. of the 1967 Int. Symposium on Electron and Photon Interactions at High Energies, CONF.-670923, UC-34-Physics, Stanford,CA, Sept. 1967, ed. S.M. Berman, pp. 109;

H. R. Quinn, Radiative Corrections To Beta Decay And Some Sum Rules For Neutrino Interactions, PhD Thesis, Stanford University, Sept. 1967, RX-327, 93pp.

[25] L. Hand, Proc. of the 1967 Int. Symposium on Electron and Photon Interactions at High Energies, CONF.670923, UC-34-Physics, Stanford,CA, Sept. 1967, ed. S.M. Berman, pp. 128.

[26] F. W. Brasse, et al., Nucl. Phys. B 39 (1972) 421.

[27] Y. Nambu, in: Preludes in Theoretical Physics, eds. A. De-Shalit, H. Fehsbach and L. van Hove (NorthHolland, Amsterdam, 1966), pp. 133.

[28] C. -N. Yang and R. L. Mills, Phys. Rev. 96 (1954) 191.

[29] M. Y. Han and Y. Nambu, Phys. Rev. 139 (1965) B1006.

[30] O. W. Greenberg, Phys. Rev. Lett. 13 (1964) 598.

[31] L. D. Faddeev and V. N. Popov, Phys. Lett. B 25 (1967) 29.

[32] G. 't Hooft, Nucl. Phys. B 33 (1971) 173.

[33] H. Fritzsch and M. Gell-Mann, Proceedings of 16th International Conference on High-Energy Physics, Batavia, Illinois, 6-13 Sep Vol. 2, J.D. Jackson, A. Roberts, R. Donaldson, eds., pp. 135 (1972), hep-ph/0208010.

[34] H. Fritzsch, M. Gell-Mann and H. Leutwyler, Phys. Lett. B 47 (1973) 365.

[35] D. J. Gross and F. Wilczek, Phys. Rev. Lett. 30 (1973) 1343.

[36] H. D. Politzer, Phys. Rev. Lett. 30 (1973) 1346.

[37] I. B. Khriplovich, Yad. Fiz. 10 (1969) 409.

[38] G. t'Hooft (1972), unpublished, cf. Proc. Colloquium on Renormalization of Yang-Mills Fields and Application to Particle Physics, Marseille, 1972, ed. C.P. Korthals-Altes; Nucl. Phys. B 254 (1985) 11.

[39] F.J. Yndurain, The Theory of Quark and Gluon Interactions, (Springer, Berlin, 1999).

[40] M. J. G. Veltman SCHOONSCHIP, version 1, Dec. 1963. 
[41] K. G. Wilson, Phys. Rev. 179 (1969) 1499.

[42] W. Zimmermann, Lectures on Elementary Particle Physics and Quantum Field Theory, Brandeis Summer Institute, Vol. 1 (MIT Press, Cambridge, 1970), p. 395.

[43] R. A. Brandt and G. Preparata, Nucl. Phys. B 27 (1972) 541;

N. H. Christ, B. Hasslacher and A. H. Mueller, Phys. Rev. D 6 (1972) 3543.

[44] Y. Frishman, Annals Phys. 66 (1971) 373.

[45] D. J. Gross and S. B. Treiman, Phys. Rev. D 4 (1971) 1059.

[46] E. C. G. Stueckelberg and A. Petermann, Helv. Phys. Acta 26 (1953) 499.

[47] K. Symanzik, Commun. Math. Phys. 18 (1970) 227.

[48] C. G. Callan, Jr., Phys. Rev. D 2 (1970) 1541.

[49] A. Petermann, Phys. Rept. 53 (1979) 157.

[50] S. D. Drell, D. J. Levy and T. -M. Yan, Phys. Rev. 187 (1969) 2159; 1035; 1617.

[51] T. -M. Yan and S. D. Drell, Phys. Rev. D 1 (1970) 2402.

[52] W.L. van Neerven, The infinite momentum frame method in field theory with some applications, PhD Thesis, Radboud University Nijmegen, 1975.

[53] T. van Ritbergen, J. A. M. Vermaseren and S. A. Larin, Phys. Lett. B 400 (1997) 379 hep-ph/9701390].

[54] M. Czakon, Nucl. Phys. B 710 (2005) 485 hep-ph/0411261.

[55] S. A. Larin, T. van Ritbergen and J. A. M. Vermaseren, Nucl. Phys. B 427 (1994) 41.

[56] S. A. Larin, P. Nogueira, T. van Ritbergen and J. A. M. Vermaseren, Nucl. Phys. B 492 (1997) 338 hep-ph/9605317.

[57] A. Retey and J. A. M. Vermaseren, Nucl. Phys. B 604 (2001) 281 hep-ph/0007294.

[58] J. Blümlein and J. A. M. Vermaseren, Phys. Lett. B 606 (2005) 130 hep-ph/0411111.

[59] S. Moch, J. A. M. Vermaseren and A. Vogt, Nucl. Phys. B 688 (2004) 101 hep-ph/0403192.

[60] A. Vogt, S. Moch and J. A. M. Vermaseren, Nucl. Phys. B 691 (2004) 129 hep-ph/0404111.

[61] J. A. M. Vermaseren, A. Vogt and S. Moch, Nucl. Phys. B 724 (2005) 3 hep-ph/0504242.

[62] P. A. Baikov and K. G. Chetyrkin, Nucl. Phys. (Proc. Suppl.) 160 (2006) 76.

[63] K. G. Chetyrkin, private communication, 2010.

[64] V. N. Velizhanin, Nucl. Phys. B 860 (2012) 288 [arXiv:1112.3954 [hep-ph]].

[65] E. Laenen, S. Riemersma, J. Smith and W. L. van Neerven, Nucl. Phys. B 392 (1993) 162; 229.

[66] S. Riemersma, J. Smith and W. L. van Neerven, Phys. Lett. B 347 (1995) 143 hep-ph/9411431.

[67] I. Bierenbaum, J. Blümlein and S. Klein, Nucl. Phys. B 820 (2009) 417 [arXiv:0904.3563 [hep-ph]].

[68] E. B. Zijlstra and W. L. van Neerven, Nucl. Phys. B 417 (1994) 61 [Erratum-ibid. B 426 (1994) 245] [Erratum-ibid. B $\mathbf{7 7 3}$ (2007) 105].

[69] R. Mertig and W. L. van Neerven, Z. Phys. C 70 (1996) 637 hep-ph/9506451. 
[70] W. Vogelsang, Phys. Rev. D 54 (1996) 2023 [hep-ph/9512218]; Nucl. Phys. B 475 (1996) 47 hep-ph/9603366.

[71] M. Buza, Y. Matiounine, J. Smith and W. L. van Neerven, Nucl. Phys. B 485 (1997) 420 hep-ph/9608342.

[72] I. Bierenbaum, J. Blümlein and S. Klein, PoS (ACAT) 070, arXiv:0706.2738 [hep-ph].

[73] A. Vogt, S. Moch, M. Rogal and J. A. M. Vermaseren, Nucl. Phys. (Proc. Suppl.) 183 (2008) 155 arXiv:0807.1238 [hep-ph]].

[74] J. A. M. Vermaseren, math-ph/0010025.

[75] M. Steinhauser, Comput. Phys. Commun. 134 (2001) 335 hep-ph/0009029.

[76] C. Schneider, J. Symbolic Comput. 43 (2008) 611, arXiv:0808.2543v1]; Ann. Comb. 9 (2005) 75; J. Differ. Equations Appl. 11 (2005) 799; Ann. Comb. 14 (4) (2010), arXiv:0808.2596]; Proceedings of the Workshop "Motives, Quantum Field Theory, and Pseudodifferential Operators", held at the Clay Mathematics Institute, Boston University, June 2-13, 2008, Clay Mathematics Proceedings 12 (2010) 285-308, Eds. A. Carey, D. Ellwood, S. Paycha, S. Rosenberg; Sém. Lothar. Combin. 56 (2007) 1, Article B56b, Habilitationsschrift JKU Linz (2007) and references therein;

J. Ablinger, J. Blümlein, S. Klein, C. Schneider, Nucl. Phys. (Proc. Suppl.) 205-206 (2010) 110 arXiv:1006.4797 [math-ph]].

[77] R. Harlander and M. Steinhauser, Prog. Part. Nucl. Phys. 43 (1999) 167 hep-ph/9812357].

[78] J. Ashman et al., [European Muon Collaboration], Phys. Lett. B 206 (1988) 364.

[79] D. Müller, D. Robaschik, B. Geyer, F. M. Dittes and J. Horejsi, Fortsch. Phys. 42 (1994) 101 hep-ph/9812448;

M. Diehl, Phys. Rept. 388 (2003) 41 hep-ph/0307382];

A. V. Belitsky and A. V. Radyushkin, Phys. Rept. 418 (2005) 1 hep-ph/0504030.

[80] X.-D. Ji, Phys. Rev. Lett. 78 (1997) 610 hep-ph/9603249.

[81] J. Blümlein and M. Klein, Nucl. Instrum. Meth. A 329 (1993) 112.

[82] F. Jaquet and A. Blondel, in: U. Amaldi al., Report From The Study Group On Detectors For Charged Current Events, (DESY, Hamburg, 1979), pp. 391.

[83] S. Bentvelsen, J. Engelen and P. Kooijman, in: W. Buchmüller and G. Ingelman (eds.), Proceedings of the Workshop on Physics at HERA, Oct. 29-30, 1991, Hamburg (DESY, Hamburg, 1992), Vol. 1, p. 23.

[84] J. Blümlein, Z. Phys. C 65 (1995) 293 [hep-ph/9403342].

[85] U. Bassler and G. Bernardi, Nucl. Instrum. Meth. A 361 (1995) 197 hep-ex/9412004.

[86] C. E. Hyde and K. de Jager, Ann. Rev. Nucl. Part. Sci. 54 (2004) 217 nucl-ex/0507001;

H. Gao, Int. J. Mod. Phys. A 20 (2005) 1595.

[87] N. Schmitz, Neutrinophysik, (Teubner, Stuttgart, 1997).

[88] R. P. Feynman, M. Kislinger and F. Ravndal, Phys. Rev. D 3 (1971) 2706;

F. Ravndal, Nuovo Cim. A 18 (1973) 385; Lett. Nuovo Cim. 3S2 (1972) 631;

D. Rein and L. M. Sehgal, Annals Phys. 133 (1981) 79.

[89] S. D. Drell and J. D. Walecka, Annals Phys. 28 (1964) 18;

T. De Forest, Jr. and J. D. Walecka, Adv. Phys. 15 (1966) 1;

E. Derman, Phys. Rev. D 19 (1979) 133. 
[90] A. Arbuzov, D. Y. Bardin, J. Blümlein, L. Kalinovskaya and T. Riemann, Comput. Phys. Commun. 94 (1996) 128 hep-ph/9511434.

[91] B. Geyer, D. Robaschik and E. Wieczorek, Fortsch. Phys. 27 (1979) 75.

[92] R. D. Tangerman and P. J. Mulders, Phys. Rev. D 51 (1995) 3357 hep-ph/9403227.

[93] J. Blümlein and A. Tkabladze, Nucl. Phys. B 553 (1999) 427 hep-ph/9812478].

[94] J. Blümlein and N. Kochelev, Nucl. Phys. B 498 (1997) 285 [hep-ph/9612318].

[95] X. -D. Ji, Nucl. Phys. B 402 (1993) 217.

[96] M. Maul, B. Ehrnsperger, E. Stein and A. Schäfer, Z. Phys. A 356 (1997) 443 hep-ph/9602377.

[97] S. Forte, M. L. Mangano and G. Ridolfi, Nucl. Phys. B 602 (2001) 585 hep-ph/0101192.

[98] W. Atwood et al., Phys.Lett. B 64 (1976) 479;

A. Bodek et al., Phys. Rev. D 20 (1979) 1471;

M. Mestayer et al., Phys.Rev. D 27 (1983) 285;

L. Whitlow, Deep inelastic structure functions from electron sctatering on hydrogen, deuterium, and at iron at $0.6 \mathrm{GeV}^{2} \leq Q^{2} \leq 30 \mathrm{GeV}^{2}, \mathrm{PhD}$ Thesis, SLAC-0357;

L. Whitlow, E. Riordan, S. Dasu, S. Rock, and A. Bodek, Phys.Lett. B 282 (1992) 475;

J. Gomez et al., Phys.Rev. D 49 (1994) 4348.

[99] S. Dasu et al., Phys. Rev. D 49 (1994) 5641.

[100] A. C. Benvenuti et al., [BCDMS Collaboration], Phys. Lett. B 223 (1989) 485.

[101] A. Benvenuti et al., BCDMS Collaboration, Phys.Lett. B 237 (1990) 592.

[102] NMC Collaboration, M. Arneodo et al., Phys.Lett. B 309 (1993) 222; Phys.Lett. B 364 (1995) 107, hep-ph/9509406; Nucl.Phys. B 487 (1997) 3, hep-ex/9611022.

[103] NMC Collaboration, M. Arneodo et al., Nucl.Phys. B 483 (1997) 3, hep-ph/9610231.

[104] F. D. Aaron et al., [H1 and ZEUS Collaboration], JHEP 1001 (2010) 109 arXiv:0911.0884 [hep-ex]].

[105] F. Eisele, Rept. Prog. Phys. 49 (1986) 233;

M. Diemoz, F. Ferroni and E. Longo, Phys. Rept. 130 (1986) 293;

T. Sloan, R. Voss and G. Smadja, Phys. Rept. 162 (1988) 45;

S. R. Mishra and F. Sciulli, Ann. Rev. Nucl. Part. Sci. 39 (1989) 259;

K. Winter, Neutrino Physics, (Cambridge, University Press, Cambridge, 1991), 670 p.

[106] L. W. Whitlow, S. Rock, A. Bodek, E. M. Riordan and S. Dasu, Phys. Lett. B 250 (1990) 193.

[107] C. Adloff et al., [H1 Collaboration], Phys. Lett. B 393 (1997) 452 hep-ex/9611017.

[108] K. Abe et al., [E143 Collaboration], Phys. Lett. B 452 (1999) 194 hep-ex/9808028.

[109] F. D. Aaron et al., Eur. Phys. J. C 71 (2011) 1579 arXiv:1012.4355 [hep-ex]].

[110] G. Moreno et al., Phys.Rev. D 43, 2815 (1991);

FNAL E866/NuSea Collaboration, R. Towell et al., Phys.Rev. D 64, 052002 (2001), hep-ex/0103030;

J. C. Webb et al., [NuSea Collaboration], hep-ex/0302019.

[111] CCFR Collaboration, A. Bazarko et al., Z.Phys. C65 (1995) 189, hep-ex/9406007;

NuTeV Collaboration, M. Goncharov et al., Phys.Rev. D 64 (2001) 112006, hep-ex/0102049;

NuTeV Collaboration, M. Tzanov et al., Phys. Rev. D 74 (2006) 012008, hep-ex/0509010;

D. A. Mason, Measurement of the strange-antistrange asymmetry at NLO in QCD from NuTeV dimuon data, PhD Thesis, FERMILAB-THESIS-2006-01. 
[112] A. D. Martin, W. J. Stirling, R. S. Thorne and G. Watt, Eur. Phys. J. C 63 (2009) 189 arXiv:0901.0002 [hep-ph]].

[113] R. D. Ball et al., Nucl. Phys. B 855 (2012) 153 [arXiv:1107.2652 [hep-ph]].

[114] C. Adloff et al., [H1 Collaboration], Eur. Phys. J. C 21 (2001) 33 hep-ex/0012053; Eur. Phys. J. C 30 (2003) 1 hep-ex/0304003]; Eur. Phys. J. C 19 (2001) 269 hep-ex/0012052;

S. Chekanov et al., [ZEUS Collaboration], Eur. Phys. J. C 21 (2001) 443 [hep-ex/0105090]; Eur. Phys. J. C 61 (2009) 223 [arXiv:0812.4620 [hep-ex]]; Eur. Phys. J. C 62 (2009) 625 [arXiv:0901.2385 [hep-ex]]; Eur. Phys. J. C 28 (2003) 175 hep-ex/0208040; Phys. Rev. D 70 (2004) 052001 hep-ex/0401003]; Eur. Phys. J. C 32 (2003) 1 [hep-ex/0307043];

J. Breitweg et al., [ZEUS Collaboration], Eur. Phys. J. C 7 (1999) 609 hep-ex/9809005]; Eur. Phys. J. C 12 (2000) 35 hep-ex/9908012;

S. Chekanov et al., [ZEUS Collaboration], Phys. Rev. D 69 (2004) 012004 hep-ex/0308068; Eur. Phys. J. C 63 (2009) 171 [arXiv:0812.3775 [hep-ex]]; Eur. Phys. J. C 65 (2010) 65 arXiv:0904.3487 [hep-ex]]; C. Adloff et al., [H1 Collaboration], Z. Phys. C 72 (1996) 593 hep-ex/9607012]; Phys. Lett. B 528 (2002) 199 [hep-ex/0108039];

A. Aktas et al., [H1 Collaboration], Eur. Phys. J. C 45 (2006) 23 hep-ex/0507081]; Eur. Phys. J. C 40 (2005) 349 [hep-ex/0411046]; Phys. Lett. B 653 (2007) 134 [arXiv:0706.3722 [hep-ex]];

S. Chekanov et al., [ZEUS Collaboration], Phys. Lett. B 547 (2002) 164 hep-ex/0208037];

Nucl. Phys. B 765 (2007) 1 [hep-ex/0608048];

M. R. Adams et al., [E665 Collaboration], Phys. Rev. D 54 (1996) 3006;

G. Onengut et al., [CHORUS Collaboration], Phys. Lett. B 632 (2006) 65;

V. M. Abazov et al., [D0 Collaboration], Phys. Rev. D 77 (2008) 011106 [arXiv:0709.4254 [hep-ex]];

D. Acosta et al., [CDF Collaboration], Phys. Rev. D 71 (2005) 051104 (hep-ex/0501023];

CDF Collaboration, J. Han et al., public note, May 2008,

http:www-cdf .fnal.gov/physics/ewk/2008/dszdy/;

T. Aaltonen et al., [CDF Collaboration], Phys. Rev. Lett. 102 (2009) 181801 [arXiv:0901.2169 [hep-ex]]; Phys. Lett. B 692 (2010) 232 [arXiv:0908.3914 [hep-ex], arXiv:0908.3914 [hep-ex]];

V. M. Abazov et al., [D0 Collaboration], Phys. Rev. D 76 (2007) 012003 hep-ex/0702025.

[115] A. Abulencia et al., [CDF - Run II Collaboration], Phys. Rev. D 75 (2007) 092006 [Erratum-ibid. D 75 (2007) 119901] [hep-ex/0701051];

T. Aaltonen et al., [CDF Collaboration], Phys. Rev. D 78 (2008) 052006 [Erratum-ibid. D 79 (2009) 119902] [arXiv:0807.2204 [hep-ex]];

V. M. Abazov et al., [D0 Collaboration], Phys. Rev. Lett. 101 (2008) 062001 arXiv:0802.2400 [hep-ex]];

Phys. Lett. B 693 (2010) 531 arXiv:1002.4594 [hep-ex]];

T. Affolder et al., [CDF Collaboration], Phys. Rev. Lett. 88 (2002) 042001 hep-ex/0108034.

[116] HERAPDF1.0, https://www.desy.de/h1zeus/combined_results/benchmark/herapdf1.0.html

[117] S. Alekhin, J. Blümlein and S. Moch, arXiv:1202.2281 [hep-ph].

[118] P. Jimenez-Delgado and E. Reya, Phys. Rev. D 79 (2009) 074023 [arXiv:0810.4274 [hep-ph]].

[119] R. D. Ball et al., Nucl. Phys. B 849 (2011) 296 [arXiv:1101.1300 [hep-ph]].

[120] P. L. Anthony et al., [E142 Collaboration], Phys. Rev. D 54 (1996) 6620 arXiv:hep-ex/9610007.

[121] K. Abe et al., [E154 Collaboration], Phys. Rev. Lett. 79 (1997) 26 arXiv:hep-ex/9705012].

[122] K. Abe et al., [E143 collaboration], Phys. Rev. D 58 (1998) 112003 arXiv:hep-ph/9802357.

[123] P. L. Anthony et al., [E155 Collaboration], Phys. Lett. B 463 (1999) 339 arXiv:hep-ex/9904002.

[124] P. L. Anthony et al., [E155 Collaboration], Phys. Lett. B 493 (2000) 19 arXiv:hep-ph/0007248. 
[125] J. Ashman et al., [European Muon Collaboration], Nucl. Phys. B 328 (1989) 1.

[126] B. Adeva et al., [Spin Muon Collaboration], Phys. Rev. D 58 (1998) 112001; Phys. Rev. D 60 (1999) 072004 [Erratum-ibid. D 62 (2000) 079902].

[127] V. Y. Alexakhin et al., [COMPASS Collaboration], Phys. Lett. B 647 (2007) 8 arXiv:hep-ex/0609038.

[128] M. G. Alekseev et al., [COMPASS Collaboration], arXiv:1001.4654 [hep-ex].

[129] E. S. Ageev et al., [COMPASS Collaboration], Phys. Lett. B 612 (2005) 154 hep-ex/0501073.

[130] X. Zheng et al., The JLAB Hall A collaboration, Phys. Rev. C70 (2004) 065207.

[131] K. V. Dharmawardane et al., [CLAS Collaboration], Phys. Lett. B 641 (2006) 11 arXiv:nucl-ex/0605028.

[132] CLAS collaboration, private communication.

[133] K. Ackerstaff et al., [HERMES Collaboration], Phys. Lett. B 404 (1997) 383 arXiv:hep-ex/9703005.

[134] A. Airapetian et al., [HERMES Collaboration], Phys. Rev. D 75 (2007) 012007 arXiv:hep-ex/0609039.

[135] D. Adams et al., [Spin Muon (SMC) Collaboration], Phys. Rev. D 56 (1997) 5330 hep-ex/9702005;

P. L. Anthony et al., [E155 Collaboration], Phys. Lett. B 553 (2003) 18 hep-ex/0204028];

A. Airapetian et al., [HERMES Collaboration], arXiv:1112.5584 [hep-ex].

[136] J. L. Abelleira Fernandez et al. [LHeC Study Group Collaboration], A Large Hadron Electron Collider at CERN: Report on the Physics and Design Concepts for Machine and Detector J. Phys. G 39 (2012) 075001 arXiv:1206.2913 [physics.acc-ph]].

[137] E. Aschenauer et al., The EIC Collaboration, A High Luminosity, High Energy Electron-lon-Collider, A White Paper Prepared for the NSAC LRP 2007.

[138] D. Boer, M. Diehl, R. Milner, R. Venugopalan, W. Vogelsang, D. Kaplan, H. Montgomery and S. Vigdor et al., arXiv:1108.1713 [nucl-th].

[139] Bernhard Riemann's Gesammelte mathematische Werke und Wissenschaftlicher Nachlass, Eds. H. Weber and R. Dedekind, (Teubner, Leipzig, 1876), p. 241;

H. Lebesgue, Ann. scient. de l'É.N.S. $3^{e}$ série, 20 (1903) 453.

[140] B. Geyer, M. Lazar and D. Robaschik, Nucl. Phys. B 559 (1999) 339 hep-th/9901090;

B. Geyer and M. Lazar, Nucl. Phys. B 581 (2000) 341 hep-th/0003080.

[141] H.D. Politzer, Nucl. Phys. B 129 (1977) 301;

D. Amati, R. Petronzio, and G. Veneziano, Nucl. Phys. B 140 (1978) 54; 146 (1978) 29;

S.B. Libby and G. Sterman, Phys. Rev. D 18 (1978) 3252, 4737;

A.H. Mueller, Phys. Rev. D 18 (1978) 3705;

J.C. Collins and G. Sterman, Nucl. Phys. B 185 (1981) 172;

J.C. Collins, D. Soper, and G. Sterman, Nucl. Phys. B 261 (1985) 104;

G.T. Bodwin, Phys. Rev. D 31 (1985) 2616.

[142] H. Mellin, Acta Soc. Sci. Fennica, 21 (1896) 1; Acta Math. 25 (1902) 139.

[143] A. Münster, Statistische Thermodynamik, (Springer, Berlin, 1956), pp. 112.

[144] M. Stratmann, A. Weber and W. Vogelsang, Phys. Rev. D 53 (1996) 138 hep-ph/9509236.

[145] J. Blümlein and N. Kochelev, Phys. Lett. B 381 (1996) 296 hep-ph/9603397.

[146] S. D. Drell and T. -M. Yan, Annals Phys. 66 (1971) 578 [Annals Phys. 281 (2000) 450]. 
[147] S. Weinberg, Phys. Rev. 150 (1966) 1313.

[148] R. M. Barnett, Phys. Rev. D 14 (1976) 70;

R. K. Ellis, R. Petronzio and G. Parisi, Phys. Lett. B 64 (1976) 97;

R. Barbieri et al., Phys. Lett. B 64 (1976) 171; Nucl. Phys. B 117 (1976) 50;

R. Brock, Phys. Rev. Lett. 44 (1980) 1027.

[149] C. Nash, Nucl. Phys. B 31 (1971) 419;

P. V. Landshoff and J. C. Polkinghorne, Phys. Rept. 5 (1972) 1.

[150] J. D. Jackson, G. G. Ross and R. G. Roberts, Phys. Lett. B 226 (1989) 159;

R. G. Roberts and G. G. Ross, Phys. Lett. B 373 (1996) 235 [hep-ph/9601235].

[151] J. A. Gracey, Phys. Lett. B 488 (2000) 175 hep-ph/0007171];

J. Blümlein, Eur. Phys. J. C 20 (2001) 683 hep-ph/0104099].

[152] W. Furmanski and R. Petronzio, Z. Phys. C 11 (1982) 293 and references therein.

[153] G. Grunberg, Phys. Rev. D 29 (1984) 2315.

[154] S. Catani, Z. Phys. C 75 (1997) 665 hep-ph/9609263].

[155] J. Blümlein, V. Ravindran and W. L. van Neerven, Nucl. Phys. B 586 (2000) 349 hep-ph/0004172.

[156] R. Tarrach, Nucl. Phys. B 183 (1981) 384;

O. Nachtmann and W. Wetzel, Nucl. Phys. B 187 (1981) 333;

N. Gray, D. J. Broadhurst, W. Grafe and K. Schilcher, Z. Phys. C 48, (1990) 673;

D. J. Broadhurst, N. Gray and K. Schilcher, Z. Phys. C 52 (1991) 111;

K. G. Chetyrkin and M. Steinhauser, Nucl. Phys. B 573, 617 (2000) arXiv:hep-ph/9911434;

Phys. Rev. Lett. 83, 4001 (1999) arXiv:hep-ph/9907509].

[157] L. F. Abbott, Nucl. Phys. B 185 (1981) 189;

A. Rebhan, Z. Phys. C 30 (1986) 309;

F. Jegerlehner and O. V. Tarasov, Nucl. Phys. B 549 (1999) 481 arXiv:hep-ph/9809485.

[158] D. W. Duke and R. G. Roberts, Phys. Rept. 120 (1985) 275.

[159] W. A. Bardeen, A. J. Buras, D. W. Duke and T. Muta, Phys. Rev. D 18 (1978) 3998.

[160] W. E. Caswell, Phys. Rev. Lett. 33 (1974) 244.

[161] D. R. T. Jones, Nucl. Phys. B 75 (1974) 531.

[162] O. V. Tarasov, A. A. Vladimirov and A. Y. Zharkov, Phys. Lett. B 93 (1980) 429.

[163] S. A. Larin and J. A. M. Vermaseren, Phys. Lett. B 303 (1993) 334 hep-ph/9302208.

[164] K. Chetyrkin et al., http://indico.cern.ch/conferenceOtherViews.py?view=standardldconf Id=154661, contribution to RADCOR 2011;

P. A. Baikov, K. G. Chetyrkin, J. H. Kühn and C. Sturm, arXiv:1207.2199 [hep-ph].

[165] J. Blümlein and J. Botts, Phys. Lett. B 325 (1994) 190 [Erratum-ibid. B 331 (1994) 450] hep-ph/9401291.

[166] K. G. Chetyrkin, B. A. Kniehl and M. Steinhauser, Phys. Rev. Lett. 79 (1997) 2184 [hep-ph/9706430.

[167] W. Bernreuther and W. Wetzel, Nucl. Phys. B 197 (1982) 228 [Erratum-ibid. B 513 (1998) 758].

[168] W. J. Marciano, Phys. Rev. D 29 (1984) 580. 
[169] E. Carlson, Thesis, Univ. Uppsala, 1914;

E.C. Titchmarsh, Theory of Functions, (Oxford University Press, Oxford, 1939), Chapt. 9.5.

[170] G. Parisi, Phys. Lett. B 43 (1973) 207; Phys. Lett. B 50 (1974) 367;

D. J. Gross, Phys. Rev. Lett. 32 (1974) 1071;

A. Zee, F. Wilczek and S. B. Treiman, Phys. Rev. D 10 (1974) 2881.

[171] D. J. Gross and F. Wilczek, Phys. Rev. D 8 (1973) 3633.

[172] D. J. Gross and F. Wilczek, Phys. Rev. D 9 (1974) 980.

[173] H. Georgi and H. D. Politzer, Phys. Rev. D 9 (1974) 416.

[174] J. A. M. Vermaseren, Int. J. Mod. Phys. A 14 (1999) 2037 hep-ph/9806280;

J. Blümlein and S. Kurth, Phys. Rev. D 60 (1999) 014018 hep-ph/9810241.

[175] E. Fermi, Z. Phys. 29 (1924) 315;

E.J. Williams, Proc. Roy. Soc. London (A) 139 (1933) 163; Phys. Rev. 45 (1934) 729; Mat. Fys. Medd. 13 (1935) 4;

C. F. von Weizsäcker, Z. Phys. 88 (1934) 612;

P. Kessler, Nuovo Cim. 17 (1960) 809;

В.Б. Берестецкий, Е.М. Лифшиц, Л.П. Питаевский, Релятивистская Квантовая Теория, Часть I, §96, (Наука, Москва, 1967); German translation, (Akademie Verlag, Berlin, 1970).

[176] K. J. Kim and Y.-S. Tsai, Phys. Rev. D 8 (1973) 3109;

V. N. Baier, V. S. Fadin and V. A. Khoze, Nucl. Phys. B 65 (1973) 381;

M. -S. Chen and P. M. Zerwas, Phys. Rev. D 12 (1975) 187.

[177] V. N. Gribov and L. N. Lipatov, Sov. J. Nucl. Phys. 15 (1972) 675 [Yad. Fiz. 15 (1972) 1218].

[178] V. N. Gribov and L. N. Lipatov, Sov. J. Nucl. Phys. 15 (1972) 438 [Yad. Fiz. 15 (1972) 781].

[179] N. Christ, Perturbation theory on the lightcone, paper \# 485 in: Proceedings of the XVI International Conference on High Energy Physics, Batavia, USA, Sept. 6-13, 1972, eds. J.D. Jackson, A. Roberts, R. Donaldson, (Fermilab, Batavia, 1972), Vol. 2, pp. 122.

[180] L. N. Lipatov, Sov. J. Nucl. Phys. 20 (1975) 94 [Yad. Fiz. 20 (1974) 181].

[181] A. P. Bukhvostov, L. N. Lipatov and N. P. Popov, Yad. Fiz. 20 (1974) 532.

[182] G. Parisi, An Introduction to Scaling Violations, LNF-76-25-P, Int. Meeting on Neutrino Physics, Flaine, March 6-12, 1976.

[183] G. Altarelli and G. Parisi, Nucl. Phys. B 126 (1977) 298.

[184] K. J. Kim and K. Schilcher, Phys. Rev. D 17 (1978) 2800.

[185] Y. L. Dokshitzer, Sov. Phys. JETP 46 (1977) 641 [Zh. Eksp. Teor. Fiz. 73 (1977) 1216].

[186] A. P. Bukhvostov, G. V. Frolov, L. N. Lipatov and E. A. Kuraev, Nucl. Phys. B 258 (1985) 601.

[187] Y.L. Dokshitzer, V.A. Khoze, A.H. Mueller, and S.I. Troyan, Basics of perturbative QCD, (Editions Frontieres, Paris, 1991).

[188] K. Sasaki, Prog. Theor. Phys. 54 (1975) 1816.

[189] M. A. Ahmed and G. G. Ross, Phys. Lett. B 56 (1975) 385.

[190] H. Ito, Prog. Theor. Phys. 54 (1975) 555. 
[191] E. G. Floratos, D. A. Ross and C. T. Sachrajda, Nucl. Phys. B 129 (1977) 66 [Erratum-ibid. B 139 (1978) 545]; Nucl. Phys. B 152 (1979) 493;

A. Gonzalez-Arroyo, C. Lopez and F. J. Yndurain, Nucl. Phys. B 153 (1979) 161; Nucl. Phys. B 159 (1979) 512;

G. Curci, W. Furmanski and R. Petronzio, Nucl. Phys. B 175 (1980) 27;

W. Furmanski and R. Petronzio, Phys. Lett. B 97 (1980) 437;

E. G. Floratos, R. Lacaze and C. Kounnas, Phys. Lett. B 98 (1981) 89; Nucl. Phys. B 192 (1981) 417;

Phys. Lett. B 98 (1981) 285;

A. Gonzalez-Arroyo and C. Lopez, Nucl. Phys. B 166 (1980) 429;

R. Hamberg and W. L. van Neerven, Nucl. Phys. B 379 (1992) 143;

R. K. Ellis and W. Vogelsang, hep-ph/9602356.

[192] S. Moch and J. A. M. Vermaseren, Nucl. Phys. B 573 (2000) 853 hep-ph/9912355.

[193] J. A. Gracey, Phys. Lett. B 322 (1994) 141 [hep-ph/9401214].

[194] J. Ablinger, J. Blümlein, S. Klein, C. Schneider and F. Wissbrock, Nucl. Phys. B 844 (2011) 26 [arXiv:1008.3347 [hep-ph]].

[195] J. Blümlein, A. Hasselhuhn, S. Klein, and C. Schneider, arXiv:1205.4184 [hep-ph].

[196] J. Blümlein, Comput. Phys. Commun. 180 (2009) 2218 arXiv:0901.3106 [hep-ph]]; arXiv:0901.0837 [math-ph]].

[197] J. Blümlein, Comput. Phys. Commun. 133 (2000) 76 hep-ph/0003100;

J. Blümlein and S. -O. Moch, Phys. Lett. B 614 (2005) 53 [hep-ph/0503188];

A. V. Kotikov and V. N. Velizhanin, hep-ph/0501274.

[198] E. Remiddi and J. A. M. Vermaseren, Int. J. Mod. Phys. A 15 (2000) 725 hep-ph/9905237.

[199] J. Blümlein, H. Böttcher and A. Guffanti, Nucl. Phys. B 774 (2007) 182 hep-ph/0607200].

[200] A.N. Vasil'ev, Yu.M. Pis'mak, and J.R. Honkonen, Theor. Math. Phys. 46 (1981) 157; 47 (1981) 291.

[201] J. A. Gracey, Phys. Lett. B 317 (1993) 415 hep-th/9309092].

[202] M. Ciuchini, S. E. Derkachov, J. A. Gracey and A. N. Manashov, Nucl. Phys. B 579 (2000) 56 hep-ph/9912221.

[203] D. B. Ali and J. A. Gracey, Phys. Lett. B 518 (2001) 188 hep-ph/0105038.

[204] J. A. Gracey, Phys. Lett. B 373 (1996) 178 hep-ph/9602214.

[205] J. F. Bennett and J. A. Gracey, Nucl. Phys. B 517 (1998) 241 hep-ph/9710364.

[206] J. A. Gracey, Nucl. Phys. (Proc. Suppl.) 51C (1996) 24 hep-ph/9604426.

[207] J. A. Gracey, Nucl. Phys. B 480 (1996) 73 hep-ph/9609301;

J. F. Bennett and J. A. Gracey, Phys. Lett. B 432 (1998) 209 hep-ph/9803446.

[208] J. A. Gracey, Nucl. Phys. B 662 (2003) 247 arXiv:hep-ph/0304113; Nucl. Phys. B 667 (2003) 242 arXiv:hep-ph/0306163. JHEP 0610 (2006) 040 arXiv:hep-ph/0609231; Phys. Lett. B 643 (2006) 374 arXiv:hep-ph/0611071.

[209] R. Hamberg, W. L. van Neerven and T. Matsuura, Nucl. Phys. B 359 (1991) 343 [Erratum-ibid. B 644 (2002) 403];

R. V. Harlander and W. B. Kilgore, Phys. Rev. Lett. 88 (2002) 201801 hep-ph/0201206. 
[210] C. Anastasiou and K. Melnikov, Nucl. Phys. B 646 (2002) 220 hep-ph/0207004;

V. Ravindran, J. Smith and W. L. van Neerven, Nucl. Phys. B 665 (2003) 325 hep-ph/0302135;

S. Catani and M. Grazzini, Phys. Rev. Lett. 98 (2007) 222002 hep-ph/0703012.

[211] G. Altarelli, R. K. Ellis and G. Martinelli, Nucl. Phys. B 157 (1979) 461.

[212] B. Humpert and W. L. van Neerven, Nucl. Phys. B 184 (1981) 225.

[213] G. T. Bodwin and J. -W. Qiu, Phys. Rev. D 41 (1990) 2755.

[214] W. Vogelsang, Z. Phys. C 50 (1991) 275.

[215] M. Glück, E. Reya, M. Stratmann and W. Vogelsang, Phys. Rev. D 53 (1996) 4775 hep-ph/9508347.

[216] E. B. Zijlstra and W. L. van Neerven, Phys. Lett. B 273 (1991) 476; Phys. Lett. B 272 (1991) 127; Nucl. Phys. B 383 (1992) 525.

[217] D. I. Kazakov and A. V. Kotikov, Nucl. Phys. B 307 (1988) 721 [Erratum-ibid. B 345 (1990) 299];

D. I. Kazakov, A. V. Kotikov, G. Parente, O. A. Sampayo and J. Sanchez Guillen, Phys. Rev. Lett. 65 (1990) 1535 [Erratum-ibid. 65 (1990) 2921];

J. Sanchez Guillen, J. Miramontes, M. Miramontes, G. Parente and O. A. Sampayo, Nucl. Phys. B 353 (1991) 337.

[218] E. B. Zijlstra and W. L. van Neerven, Phys. Lett. B 297 (1992) 377.

[219] S. A. Larin and J. A. M. Vermaseren, Z. Phys. C 57 (1993) 93.

[220] J. Blümlein, M. Kauers, S. Klein and C. Schneider, Comput. Phys. Commun. 180 (2009) 2143 arXiv:0902.4091 [hep-ph]].

[221] J. Blümlein and V. Ravindran, Nucl. Phys. B 716 (2005) 128 hep-ph/0501178; Nucl. Phys. B 749 (2006) 1 hep-ph/0604019].

[222] S. Moch and M. Rogal, Nucl. Phys. B 782 (2007) 51 [arXiv:0704.1740 [hep-ph]];

S. Moch, M. Rogal and A. Vogt, Nucl. Phys. B 790 (2008) 317 [arXiv:0708.3731 [hep-ph]];

S. Moch, J. A. M. Vermaseren and A. Vogt, Nucl. Phys. B 813 (2009) 220 [arXiv:0812.4168 [hep-ph]].

[223] J. Blümlein, Comput. Phys. Commun. 159 (2004) 19 hep-ph/0311046.

[224] L. W. Mo and Y. -S. Tsai, Rev. Mod. Phys. 41 (1969) 205.

[225] D. Y. Bardin, O. M. Fedorenko and N. M. Shumeiko, J. Phys. G 7 (1981) 1331.

[226] M. Consoli and M. Greco, Nucl. Phys. B 186 (1981) 519.

[227] W. J. Marciano and A. Sirlin, Phys. Rev. D 22 (1980) 2695 [Erratum-ibid. D 31 (1985) 213]; Nucl. Phys.

B 189 (1981) 442;

S. Sarantakos, A. Sirlin and W. J. Marciano, Nucl. Phys. B 217 (1983) 84;

C. H. Llewellyn Smith and J. F. Wheater, Phys. Lett. B 105 (1981) 486; Nucl. Phys. B 208 (1982) 27 [Erratum-ibid. B 226 (1983) 547];

E. A. Paschos and M. Wirbel, Nucl. Phys. B 194 (1982) 189;

M. Wirbel, Z. Phys. C 14 (1982) 293;

I. Liede, Nucl. Phys. B 229 (1983) 499;

D. Y. Bardin and V. A. Dokuchaeva, JINR-E2-86-260.

[228] K.-P. O. Diener, S. Dittmaier and W. Hollik, Phys. Rev. D 69 (2004) 073005 hep-ph/0310364;

A. B. Arbuzov, D. Y. Bardin and L. V. Kalinovskaya, JHEP 0506 (2005) 078 arXiv:hep-ph/0407203;

K.-P. O. Diener, S. Dittmaier and W. Hollik, Phys. Rev. D 72 (2005) 093002 hep-ph/0509084;

K. Park, U. Baur and D. Wackeroth, arXiv:0910.5013 [hep-ph]. 
[229] G. P. Zeller et al., [NuTeV Collaboration], Phys. Rev. Lett. 88 (2002) 091802 [Erratum-ibid. 90 (2003) 239902] hep-ex/0110059.

[230] T. Kinoshita, J. Math. Phys. 3 (1962) 650;

T. D. Lee and M. Nauenberg, Phys. Rev. 133 (1964) B1549.

[231] A. De Rujula, R. Petronzio and A. Savoy-Navarro, Nucl. Phys. B 154 (1979) 394.

[232] M. Böhm and H. Spiesberger, Nucl. Phys. B 294 (1987) 1081.

[233] M. Böhm and H. Spiesberger, Nucl. Phys. B 304 (1988) 749.

[234] D. Y. Bardin, C. Burdik, P. K. Christova and T. Riemann, JINR-E2-87-595.

[235] D. Y. Bardin, C. Burdik, P. C. Christova and T. Riemann, Z. Phys. C 42 (1989) 679.

[236] D. Y. Bardin, K. C. Burdik, P. K. Christova and T. Riemann, Z. Phys. C 44 (1989) 149.

[237] E. A. Kuraev, N. P. Merenkov and V. S. Fadin, Sov. J. Nucl. Phys. 47 (1988) 1009 [Yad. Fiz. 47 (1988) 1593].

[238] W. Beenakker, F. Berends, and W. van Neerven, in: Proc. of the Workshop on Electroweak Radiative Corrections, Ringberg, Germany, ed. J. Kühn, (Springer, Berlin, 1989), p. 3.

[239] J. Blümlein, Z. Phys. C 47 (1990) 89.

[240] J. Blümlein, Phys. Lett. B 271 (1991) 267.

[241] J. Blümlein, HELIOS 1.00, PHE 91-16, Proc. of the 1992 HERA Workshop, Vol. 3, eds. W. Buchmüller and G. Ingelman, (DESY, Hamburg, 1992), p. 1269.

[242] G. Montagna, O. Nicrosini and L. Trentadue, Nucl. Phys. B 357 (1991) 390.

[243] J. Kripfganz and H. J. Möhring, Z. Phys. C 38 (1988) 653.

[244] J. Kripfganz, H. J. Möhring and H. Spiesberger, Z. Phys. C 49 (1991) 501.

[245] A. A. Akhundov, D. Y. Bardin, L. Kalinovskaya and T. Riemann, Phys. Lett. B 301 (1993) 447 hep-ph/9507278.

[246] A. A. Akhundov, D. Y. Bardin, L. Kalinovskaya and T. Riemann, Fortsch. Phys. 44 (1996) 373 hep-ph/9407266.

[247] D. Y. Bardin, P. Christova, L. Kalinovskaya and T. Riemann, Phys. Lett. B 357 (1995) 456 hep-ph/9504423.

[248] H. Spiesberger, Nucl. Phys. B 349 (1991) 109.

[249] A. Kwiatkowski, H. Spiesberger and H. J. Möhring, Comput. Phys. Commun. 69 (1992) 155.

[250] J. Blümlein, G. Levman and H. Spiesberger, J. Phys. G 19 (1993) 1695.

[251] J. Blümlein and H. Kawamura, Phys. Lett. B 553 (2003) 242 hep-ph/0211191.

[252] J. Kripfganz and H. Perlt, Z. Phys. C 41 (1988) 319.

[253] H. Spiesberger, Phys. Rev. D 52 (1995) 4936 hep-ph/9412286;

M. Roth and S. Weinzierl, Phys. Lett. B 590 (2004) 190 hep-ph/0403200].

[254] D. Y. Bardin, J. Blümlein, P. Christova and L. Kalinovskaya, Nucl. Phys. B 506 (1997) 295 hep-ph/9612435. 
[255] V. S. Fadin, In: Conference Proceedings No. 201, QED Structure Functions, Ann Arbor, 1989, Ed. G. Bonvicini, (APS, New York, 1989), pp. 118.

[256] I. V. Akushevich, A. N. Ilyichev and N. M. Shumeiko, J. Phys. G 24 (1998) 1995 hep-ph/9804361;

I. V. Akushevich, O. F. Filoti, A. Ilyichev and N. Shumeiko, arXiv:1104.0039 [hep-ph].

[257] M. Skrzypek, Acta Phys. Polon. B 23 (1992) 135.

[258] M. Jezabek, Z. Phys. C 56 (1992) 285.

[259] M. Przybycien, Acta Phys. Polon. B 24 (1993) 1105 hep-th/9511029;

A. B. Arbuzov, Phys. Lett. B 470 (1999) 252 hep-ph/9908361.

[260] J. Blümlein and H. Kawamura, Acta Phys. Polon. B 33 (2002) 3719 hep-ph/0207259]; Nucl. Phys. B 708 (2005) 467 hep-ph/0409289.

[261] J. Blümlein and H. Kawamura, Eur. Phys. J. C 51 (2007) 317 hep-ph/0701019.

[262] J. Blümlein, S. Riemersma and A. Vogt, Eur. Phys. J. C 1 (1998) 255 hep-ph/9611214.

[263] E. Witten, Nucl. Phys. B 104 (1976) 445;

J. Babcock, D. W. Sivers and S. Wolfram, Phys. Rev. D 18 (1978) 162;

M. A. Shifman, A. I. Vainshtein and V. I. Zakharov, Nucl. Phys. B 136 (1978) 157 [Yad. Fiz. 27 (1978) 455];

J. P. Leveille and T. J. Weiler, Nucl. Phys. B 147 (1979) 147.

[264] A. D. Watson, Z. Phys. C 12 (1982) 123.

[265] J. Blümlein, V. Ravindran and W. L. van Neerven, Phys. Rev. D 68 (2003) 114004 hep-ph/0304292.

[266] S. Wandzura and F. Wilczek, Phys. Lett. B 72 (1977) 195.

[267] S. I. Alekhin and J. Blümlein, Phys. Lett. B 594 (2004) 299 [hep-ph/0404034.

[268] J. C. Collins, Phys. Rev. D 58 (1998) 094002 hep-ph/9806259.

[269] J. Blümlein, A. De Freitas and W. van Neerven, Nucl. Phys. B 855 (2012) 508 [arXiv:1107.4638 [hep-ph]].

[270] I. Bierenbaum, J. Blümlein and S. Klein, Nucl. Phys. B 780 (2007) 40 hep-ph/0703285.

[271] I. Bierenbaum, J. Blümlein, S. Klein and C. Schneider, Nucl. Phys. B 803 (2008) 1 arXiv:0803.0273 [hep-ph]].

[272] T. Gottschalk, Phys. Rev. D 23 (1981) 56;

M. Glück, S. Kretzer and E. Reya, Phys. Lett. B 380 (1996) 171 [Erratum-ibid. B 405 (1997) 391] hep-ph/9603304;

J. Blümlein, A. Hasselhuhn, P. Kovacikova and S. Moch, Phys. Lett. B 700 (2011) 294 arXiv:1104.3449 [hep-ph]];

M. Buza and W. L. van Neerven, Nucl. Phys. B 500 (1997) 301 hep-ph/9702242.

[273] I. Bierenbaum, J. Blümlein and S. Klein, PoS DIS2010 (2010) 148 [arXiv:1008.0792 [hep-ph]].

[274] J. Ablinger, J. Blümlein, A. Hasselhuhn, S. Klein, C. Schneider, F. Wißbrock, Nucl. Phys. B 864 (2012) 52 arXiv:1206.2252 [hep-ph]].

[275] J. Ablinger, J. Blümlein, and C. Schneider, in preparation.

[276] J. Blümlein, A. De Freitas, W. L. van Neerven and S. Klein, Nucl. Phys. B 755 (2006) 272 arXiv:hep-ph/0608024. 
[277] J. Blümlein, S. Klein and B. Tödtli, Phys. Rev. D 80 (2009) 094010 [arXiv:0909.1547 [hep-ph]].

[278] S. Alekhin and S. Moch, Phys. Lett. B 699 (2011) 345 [arXiv:1011.5790 [hep-ph]].

[279] E. Laenen and S.-O. Moch, Phys. Rev. D 59 (1999) 034027 hep-ph/9809550];

N. A. Lo Presti, H. Kawamura, S. Moch and A. Vogt, PoS DIS2010 (2010) 163 [arXiv:1008.0951 [hep-ph]].

[280] T. O. Eynck and S .Moch, Phys. Lett. B 495 (2000) 87 hep-ph/0008108.

[281] M. Glück, E. Reya and M. Stratmann, Nucl. Phys. B 422 (1994) 37.

[282] S. Alekhin, J. Blümlein, S. Klein and S. Moch, Phys. Rev. D 81 (2010) 014032 [arXiv:0908.2766 [hep-ph]].

[283] M. Buza, Y. Matiounine, J. Smith and W. L. van Neerven, Eur. Phys. J. C 1 (1998) 301 hep-ph/9612398].

[284] I. Bierenbaum, J. Blümlein and S. Klein, Phys. Lett. B 672 (2009) 401 [arXiv:0901.0669 [hep-ph]].

[285] M. A. G. Aivazis, F. I. Olness and W. -K. Tung, Phys. Rev. D 50 (1994) 3085 hep-ph/9312318];

M. A. G. Aivazis, J. C. Collins, F. I. Olness and W. -K. Tung, Phys. Rev. D 50 (1994) 3102; hep-ph/9312319;

W. -K. Tung, S. Kretzer and C. Schmidt, J. Phys. G 28 (2002) 983 hep-ph/0110247.

[286] R. S. Thorne and R. G. Roberts, Phys. Rev. D 57 (1998) 6871 hep-ph/9709442];

R. S. Thorne, Phys. Rev. D 73 (2006) 054019 hep-ph/0601245].

[287] S. Forte, E. Laenen, P. Nason and J. Rojo, Nucl. Phys. B 834 (2010) 116 [arXiv:1001.2312 [hep-ph]].

[288] J. Blümlein and W. L. van Neerven, Phys. Lett. B 450 (1999) 417 hep-ph/9811351.

[289] J. Ablinger, J. Blümlein, S. Klein, C. Schneider and F. Wissbrock, arXiv:1106.5937 [hep-ph];

J. Ablinger, et al., arXiv:1202.2700 [hep-ph].

[290] R. Harlander, T. Seidensticker, M. Steinhauser, Phys. Lett. B426 (1998) 125, hep-ph/9712228;

T. Seidensticker, hep-ph/9905298.

[291] O. Nachtmann, Nucl. Phys. B 63 (1973) 237.

[292] S. Wandzura, Nucl. Phys. B 122 (1977) 412.

[293] H. Georgi and H. D. Politzer, Phys. Rev. D 14 (1976) 1829.

[294] I. Schienbein et al., J. Phys. G 35 (2008) 053101 [arXiv:0709.1775 [hep-ph]].

[295] J. Blümlein and H. Böttcher, arXiv:1207.3170 [hep-ph].

[296] K. Bitar, P. W. Johnson and W. K. Tung, Phys. Lett. B 83 (1979) 114.

[297] J. L. Miramontes and J. Sanchez Guillen, Z. Phys. C 41 (1988) 247.

[298] A. Accardi and J. -W. Qiu, JHEP 0807 (2008) 090 [arXiv:0805.1496 [hep-ph]].

[299] A. Piccione and G. Ridolfi, Nucl. Phys. B 513 (1998) 301 hep-ph/9707478.

[300] A. V. Belitsky and D. Müller, Phys. Lett. B 507 (2001) 173 hep-ph/0102224.

[301] B. Geyer, D. Robaschik and J. Eilers, Nucl. Phys. B 704 (2005) 279 [hep-ph/0407300.

[302] J. Blümlein, D. Robaschik and B. Geyer, Eur. Phys. J. C 61 (2009) 279 arXiv:0812.1899 [hep-ph]].

[303] J. Blümlein and A. Vogt, Phys. Rev. D 58 (1998) 014020 hep-ph/9712546.

[304] R. K. Ellis, Z. Kunszt and E. M. Levin, Nucl. Phys. B 420 (1994) 517 [Erratum-ibid. B 433 (1995) 498]. 
[305] M. Glück, E. Reya and A. Vogt, Z. Phys. C 48 (1990) 471.

[306] A. Vogt, Comput. Phys. Commun. 170 (2005) 65 hep-ph/0408244.

[307] G. P. Salam and J. Rojo, Comput. Phys. Commun. 180 (2009) 120 [arXiv:0804.3755 [hep-ph]].

[308] M. Botje, Comput. Phys. Commun. 182 (2011) 490 [arXiv:1005.1481 [hep-ph]], http://www.nikhef.nl/ h24/qcdnum/.

[309] S. Alekhin et al., http://www-zeuthen.desy.de/ alekhin/OPENQCDRAD/

[310] F. J. Yndurain, Phys. Lett. B 74 (1978) 68;

G. Parisi and N. Sourlas, Nucl. Phys. B 151 (1979) 421;

R. Kobayashi, M. Konuma, S. Kumano, Comput. Phys. Commun. 86 (1995) 264, hep-ph/9409289];

Z. Phys. C31 (1986) 151;

J. Chyla and J. Rames, Z. Phys. C 31 (1986) 151;

V. G. Krivokhizhin et al.. Z. Phys. C 36 (1987) 51; Z. Phys. C 48 (1990) 347;

J. Blümlein, M. Klein, G. Ingelman and R. Rückl, Z. Phys. C 45 (1990) 501.

[311] W. Furmanski, R. Petronzio, Nucl. Phys. B 195 (1982) 237.

[312] S. Forte, L. Garrido, J. I. Latorre and A. Piccione, JHEP 0205 (2002) 062 hep-ph/0204232.

[313] A. De Roeck and R. S. Thorne, Prog. Part. Nucl. Phys. 66 (2011) 727 [arXiv:1103.0555 [hep-ph]].

[314] E. Perez and E. Rizvi, arXiv:1208.1178 [hep-ex].

[315] A. J. Buras and K. J. F. Gaemers, Nucl. Phys. B 132 (1978) 249.

[316] M. Glück, E. Hoffmann and E. Reya, Z. Phys. C 13 (1982) 119.

[317] D. W. Duke and J. F. Owens, Phys. Rev. D 30 (1984) 49.

[318] E. Eichten, I. Hinchliffe, K. D. Lane and C. Quigg, Rev. Mod. Phys. 56 (1984) 579 [Addendum-ibid. 58 (1986) 1065].

[319] M. Glück, E. Reya and A. Vogt, Z. Phys. C 53 (1992) 127; 67 (1995) 433; Eur. Phys. J. C 5 (1998) 461 hep-ph/9806404.

[320] A. D. Martin, R. G. Roberts and W. J. Stirling, Phys. Rev. D 37 (1988) 1161.

[321] W. -K. Tung, Nucl. Phys. B 315 (1989) 378;

J. G. Morfin and W. -K. Tung, Z. Phys. C 52 (1991) 13.

[322] H. -L. Lai, et al., Phys. Rev. D 82 (2010) 074024 arXiv:1007.2241 [hep-ph]] and NNLO analysis in preparation.

[323] HERAPDF collab. https://www.desy.de/h1zeus/combined results/proton structure/Fits/HERAPDF1.0 NNLO 1145.LHgrid.gz

[324] M. Glück, P. Jimenez-Delgado and E. Reya, Eur. Phys. J. C 53 (2008) 355 [arXiv:0709.0614 [hep-ph]].

[325] S. Alekhin, K. Melnikov and F. Petriello, Phys. Rev. D 74 (2006) 054033 arXiv:hep-ph/0606237.

[326] G. S. Bali, S. Collins, M. Deka, B. Glaessle, M. Göckeler, J. Najjar, A. Nobile and D. Pleiter et al., arXiv:1207.1110 [hep-lat].

[327] R. Horsley et al. [QCDSF and UKQCD Collaborations], Phys. Lett. B 714 (2012) 312 arXiv:1205.6410 [hep-lat]]. 
[328] D. B. Renner, arXiv:1002.0925 [hep-lat].

[329] J. Blümlein and H. Böttcher, Nucl. Phys. B 841 (2010) 205 [arXiv:1005.3113 [hep-ph]].

[330] M. Glück, E. Reya, M. Stratmann and W. Vogelsang, Phys. Rev. D 63 (2001) 094005 hep-ph/0011215].

[331] D. de Florian, R. Sassot, M. Stratmann and W. Vogelsang, Phys. Rev. D 80 (2009) 034030 arXiv:0904.3821 [hep-ph]].

[332] M. Hirai et al. [Asymmetry Analysis Collaboration], Nucl. Phys. B 813 (2009) 106 arXiv:0808.0413 [hep-ph]].

[333] E. Leader, A. V. Sidorov and D. B. Stamenov, Eur. Phys. J. C 23 (2002) 479 hep-ph/0111267.

[334] J. Blümlein and H. Böttcher, Nucl. Phys. B 636 (2002) 225 hep-ph/0203155.

[335] G. P. Ramsey, Prog. Part. Nucl. Phys. 39 (1997) 599 hep-ph/9702227.

[336] G. Altarelli, R. D. Ball, S. Forte and G. Ridolfi, Nucl. Phys. B 496 (1997) 337 hep-ph/9701289;

G. Altarelli, S. Forte and G. Ridolfi, Nucl. Phys. B 534 (1998) 277 hep-ph/9806345.

[337] Y. Goto et al. [Asymmetry Analysis Collaboration], Phys. Rev. D 62 (2000) 034017 hep-ph/0001046.

[338] B. Lampe and E. Reya, Phys. Rept. 332 (2000) 1 hep-ph/9810270.

[339] S. E. Kuhn, J. -P. Chen and E. Leader, Prog. Part. Nucl. Phys. 63 (2009) 1 arXiv:0812.3535 [hep-ph]].

[340] C. R. V. Bourrely, J. Soffer and F. Buccella, Eur. Phys. J. C 41 (2005) 327 hep-ph/0502180; Phys. Lett. B 648 (2007) 39 [hep-ph/0702221];

J. Soffer, AIP Conf. Proc. 1350 (2011) 305 [arXiv:1011.3209 [hep-ph]].

[341] A. Airapetian et al. [HERMES Collaboration], Phys. Rev. D 71 (2005) 012003 hep-ex/0407032.

[342] E. Leader, A. V. Sidorov and D. B. Stamenov, Phys. Rev. D 82 (2010) 114018 [arXiv:1010.0574 [hep-ph]].

[343] M. Glück, E. Reya and C. Schuck, Nucl. Phys. B 754 (2006) 178 hep-ph/0604116.

[344] A. D. Martin, W. J. Stirling, R. S. Thorne and G. Watt, Eur. Phys. J. C 64 (2009) 653 arXiv:0905.3531 [hep-ph]].

[345] R. D. Ball, et al. Phys. Lett. B 707 (2012) 66 [arXiv:1110.2483 [hep-ph]].

[346] P. Nadolsky, http://indico.desy.de/conferenceDisplay.py?conf Id=4211 (unpublished).

[347] S. Bethke, et al., Workshop on Precision Measurements of $\alpha_{s}$, arXiv:1110.0016 [hep-ph].

[348] G. Dissertori, A. Gehrmann-De Ridder, T. Gehrmann, E. W. N. Glover, G. Heinrich and H. Stenzel, Phys. Rev. Lett. 104 (2010) 072002 arXiv:0910.4283 [hep-ph]].

[349] P. A. Baikov, K. G. Chetyrkin and J. H. Kühn, Phys. Rev. Lett. 101 (2008) 012002 arXiv:0801.1821 [hep-ph]].

[350] P. A. Baikov, K. G. Chetyrkin, J. H. Kühn and J. Rittinger, Phys. Rev. Lett. 108 (2012) 222003 arXiv:1201.5804 [hep-ph]].

[351] T. Gehrmann, M. Jaquier and G. Luisoni, Eur. Phys. J. C 67 (2010) 57 [arXiv:0911.2422 [hep-ph]].

[352] R. Abbate, M. Fickinger, A. H. Hoang, V. Mateu and I. W. Stewart, Phys. Rev. D 83 (2011) 074021 arXiv:1006.3080 [hep-ph]]. 
[353] S. Aoki et al. [PACS-CS Collaboration], JHEP 0910 (2009) 053 [arXiv:0906.3906 [hep-lat]];

C. McNeile, C. T. H. Davies, E. Follana, K. Hornbostel and G. P. Lepage, Phys. Rev. D 82 (2010) 034512 arXiv:1004.4285 [hep-lat]];

B. Blossier, P. Boucaud, M. Brinet, F. De Soto, X. Du, V. Morenas, O. Pene and K. Petrov et al., arXiv:1201.5770 [hep-ph];

P. Fritzsch, F. Knechtli, B. Leder, M. Marinkovic, S. Schaefer, R. Sommer and F. Virotta, arXiv:1205.5380 [hep-lat];

A. Bazavov, N. Brambilla, X. Garcia i Tormo, P. Petreczky, J. Soto and A. Vairo, arXiv:1205.6155[hep-ph];

X. Garcia i Tormo, arXiv:1208.4850.

[354] B. Malaescu and P. Starovoitov, Eur. Phys. J. C 72 (2012) 2041 [arXiv:1203.5416 [hep-ph]].

[355] R. Frederix, S. Frixione, K. Melnikov and G. Zanderighi, JHEP 1011 (2010) 050 arXiv:1008.5313 [hep$\mathrm{ph}]$.

[356] V. S. Fadin, E. A. Kuraev and L. N. Lipatov, Phys. Lett. B 60 (1975) 50;

L. N. Lipatov, Sov. J. Nucl. Phys. 23 (1976) 338 [Yad. Fiz. 23 (1976) 642];

E. A. Kuraev, L. N. Lipatov and V. S. Fadin, Sov. Phys. JETP 45 (1977) 199 [Zh. Eksp. Teor. Fiz. 72 (1977) 377];

I. I. Balitsky and L. N. Lipatov, Sov. J. Nucl. Phys. 28 (1978) 822 [Yad. Fiz. 28 (1978) 1597];

L. N. Lipatov, Sov. Phys. JETP 63 (1986) 904 [Zh. Eksp. Teor. Fiz. 90 (1986) 1536];

M. Ciafaloni, Nucl. Phys. B 296 (1988) 49.

[357] L. V. Gribov, E. M. Levin and M. G. Ryskin, Nucl. Phys. B 188 (1981) 555;

A. H. Mueller and J. W. Qiu, Nucl. Phys. B 268 (1986) 427.

[358] R.J. Glauber, Lectures in Theoretical Physics, (Interscience Publishers, 1959).

[359] J. Kwiecinski, Z. Phys. C 29 (1985) 147.

[360] J. C. Collins and J. Kwiecinski, Nucl. Phys. B 335 (1990) 89;

J. Bartels, G. A. Schuler and J. Blümlein, Z. Phys. C 50 (1991) 91 [Nucl. Phys. Proc. Suppl. 18C (1991) 147];

M. Altmann, M. Glück and E. Reya, Phys. Lett. B 285 (1992) 359.

[361] L. McLerran, (ed.), J. Dunlop, (ed.), D. Morrison, (ed.) and R. Venugopalan, (ed.), Saturation the color glass condensate and the glasma: What have we learned from RHIC?, Proceedings, Workshop, Upton, Brookhaven, USA, May 10-12, 2010, 256 pp.

[362] G. Marchesini, in: QCD at 200 TeV, ed. by L. Ciffarelli and Yu.L. Dokshitser, (Plenum Press, New York, 1992) pp. 183 and references therein.

[363] J. Blümlein and A. Vogt, Phys. Lett. B 370 (1996) 149 [hep-ph/9510410]; Acta Phys. Polon. B 27 (1996) 1309 hep-ph/9603450.

[364] R. Kirschner and L. N. Lipatov, Nucl. Phys. B 213 (1983) 122.

[365] J. Bartels, B. I. Ermolaev and M. G. Ryskin, Z. Phys. C 72 (1996) 627 hep-ph/9603204.

[366] J. Blümlein and A. Vogt, Phys. Lett. B 386 (1996) 350 hep-ph/9606254.

[367] Y. Kiyo, J. Kodaira and H. Tochimura, Z. Phys. C 74 (1997) 631 hep-ph/9701365.

[368] B. I. Ermolaev, S. I. Manaenkov and M. G. Ryskin, Z. Phys. C 69 (1996) 259 hep-ph/9502262;

J. Bartels, B. I. Ermolaev and M. G. Ryskin, Z. Phys. C 70 (1996) 273 hep-ph/9507271].

[369] T. Jaroszewicz, Acta Phys. Polon. B 11 (1980) 965; Phys. Lett. B 116 (1982) 291. 
[370] R. K. Ellis, F. Hautmann and B. R. Webber, Phys. Lett. B 348 (1995) 582 hep-ph/9501307.

[371] J. Blümlein, Proc. QCD and high energy hadronic interactions, Les Arcs, ed. Tran Than Van, pp. 191, hep-ph/9506446.

[372] J. Kwiecinski, Z. Phys. C 29 (1985) 561.

[373] J. Blümlein, S. Riemersma and A. Vogt, Nucl. Phys. (Proc. Suppl.) 51C (1996) 30 hep-ph/9608470. Acta Phys. Polon. B 28 (1997) 577 hep-ph/9610427.

[374] S. Catani and F. Hautmann, Nucl. Phys. B 427 (1994) 475 hep-ph/9405388].

[375] R. Kirschner and M. Segond, Eur. Phys. J. C 68 (2010) 425 [arXiv:0910.5443 [hep-ph]].

[376] V. S. Fadin and L. N. Lipatov, Phys. Lett. B 429 (1998) 127 hep-ph/9802290];

V. S. Fadin, hep-ph/9807528.

[377] G. Camici and M. Ciafaloni, Nucl. Phys. B 496 (1997) 305 [Erratum-ibid. B 607 (2001) 431] hep-ph/9701303. Phys. Lett. B 430 (1998) 349 hep-ph/9803389.

[378] M. Ciafaloni, Phys. Lett. B 356 (1995) 74 hep-ph/9507307].

[379] J. Blumlein, J. Phys. G 19 (1993) 1623.

[380] J. Blümlein, V. Ravindran, W. L. van Neerven and A. Vogt, The Unpolarized gluon anomalous dimension at small $x$, DIS98, 6th International Workshop on Deep Inelastic Scattering and QCD, Brussels, Belgium, April, 1998, ed. by Gh. Coremans and R. Rosen (World Scientific, Singapore, 1998), 211-216 hep-ph/9806368.

[381] G. Altarelli, R. D. Ball and S. Forte, Nucl. Phys. B 799 (2008) 199 arXiv:0802.0032 [hep-ph]].

[382] V. S. Fadin, R. Fiore and M. I. Kotsky, Phys. Lett. B 387 (1996) 593 hep-ph/9605357.

[383] J. Blümlein, V. Ravindran and W. L. van Neerven, Phys. Rev. D 58 (1998) 091502 hep-ph/9806357.

[384] I. A. Korchemskaya and G. P. Korchemsky, Phys. Lett. B 387 (1996) 346 hep-ph/9607229]. [385]

[385] R. D. Ball and S. Forte, Phys. Lett. B 465 (1999) 271 hep-ph/9906222.

[386] G. Altarelli, R. D. Ball and S. Forte, Nucl. Phys. B 742 (2006) 1 hep-ph/0512237] and references quoted therein.

[387] M. Ciafaloni, D. Colferai, G. P. Salam and A. M. Stasto, Phys. Lett. B 635 (2006) 320 hep-ph/0601200.

[388] M. Ciafaloni, D. Colferai, G. P. Salam and A. M. Stasto, JHEP 0708 (2007) 046 arXiv:0707.1453 [hep$\mathrm{ph}]$ ] and references quoted therein.

[389] C. Lovelace, Phys. Lett. B 55 (1975) 187; Nucl. Phys. B 95 (1975) 12.

[390] J. Blümlein and W. L. van Neerven, Phys. Lett. B 450 (1999) 412 hep-ph/9811519.

[391] S. Moch and A. Vogt, JHEP 0911 (2009) 099 [arXiv:0909.2124 [hep-ph]].

[392] A. A. Almasy, G. Soar and A. Vogt, JHEP 1103 (2011) 030 [arXiv:1012.3352 [hep-ph]].

[393] J. Kodaira and L. Trentadue, Phys. Lett. B 112 (1982) 66.

[394] G. F. Sterman, Nucl. Phys. B 281 (1987) 310;

S. Catani and L. Trentadue, Nucl. Phys. B 327 (1989) 323; Nucl. Phys. B 353 (1991) 183; S. Catani, M. L. Mangano, P. Nason and L. Trentadue, Nucl. Phys. B 478 (1996) 273 [hep-ph/9604351;

H. Contopanagos, E. Laenen and G. F. Sterman, Nucl. Phys. B 484 (1997) 303 hep-ph/9604313. 
[395] A. Vogt, Phys. Lett. B 471 (1999) 97 hep-ph/9910545].

[396] S. Moch, J. A. M. Vermaseren and A. Vogt, Nucl. Phys. B 726 (2005) 317 hep-ph/0506288 and Erratum; V. Ravindran, Nucl. Phys. B 752 (2006) 173 hep-ph/0603041.

[397] E. Laenen, L. Magnea and G. Stavenga, Phys. Lett. B 669 (2008) 173 [arXiv:0807.4412 [hep-ph]].

[398] G. Soar, S. Moch, J. A. M. Vermaseren and A. Vogt, Nucl. Phys. B 832 (2010) 152 arXiv:0912.0369 [hep-ph]].

[399] E. Laenen, G. Stavenga and C. D. White, JHEP 0903 (2009) 054 [arXiv:0811.2067 [hep-ph]];

E. Laenen, L. Magnea, G. Stavenga and C. D. White, JHEP 1101 (2011) 141 [arXiv:1010.1860 [hep-ph]].

[400] V. Ravindran and W. L. van Neerven, Nucl. Phys. B 605 (2001) 517 hep-ph/0102280].

[401] S. L. Adler, Phys. Rev. 143 (1966) 1144.

[402] J. D. Bjorken, Phys. Rev. 163 (1967) 1767.

[403] S. A. Larin, F. V. Tkachov and J. A. M. Vermaseren, Phys. Rev. Lett. 66 (1991) 862.

[404] D. J. Gross and C. H. Llewellyn Smith, Nucl. Phys. B 14 (1969) 337.

[405] S. A. Larin and J. A. M. Vermaseren, Phys. Lett. B 259 (1991) 345.

[406] P. A. Baikov, K. G. Chetyrkin, J. H. Kuhn and J. Rittinger, Phys. Lett. B 714 (2012) 62 arXiv:1206.1288 [hep-ph]].

[407] J. D. Bjorken, Phys. Rev. D 1 (1970) 1376.

[408] P. A. Baikov, K. G. Chetyrkin and J. H. Kühn, Phys. Rev. Lett. 104 (2010) 132004 arXiv:1001.3606 [hep-ph]].

[409] S. B. Gerasimov, Sov. J. Nucl. Phys. 2 (1966) 430 [Yad. Fiz. 2 (1965) 598].

[410] S. D. Drell and A. C. Hearn, Phys. Rev. Lett. 16 (1966) 908.

[411] D. Drechsel, S. S. Kamalov and L. Tiator, Phys. Rev. D 63 (2001) 114010 hep-ph/0008306.

[412] D. Drechsel and L. Tiator, Ann. Rev. Nucl. Part. Sci. 54 (2004) 69 nucl-th/0406059.

[413] H. Burkhardt and W. N. Cottingham, Annals Phys. 56 (1970) 453.

[414] R. L. Jaffe, Comments Nucl. Part. Phys. 19 (1990) 239.

[415] B.L. Ioffe, V.A. Khoze, and L.N. Lipatov, Hard Processes, Vol. 1 (North-Holland, Amsterdam, 1984).

[416] R. L. Heimann, Nucl. Phys. B 64 (1973) 429.

[417] J. Kodaira, S. Matsuda, T. Uematsu and K. Sasaki, Phys. Lett. B 345 (1995) 527 hep-ph/9408353.

[418] A. V. Efremov, O. V. Teryaev and E. Leader, Phys. Rev. D 55 (1997) 4307 hep-ph/9607217.

[419] J. R. Ellis and R. L. Jaffe, Phys. Rev. D 9 (1974) 1444 [Erratum-ibid. D 10 (1974) 1669].

[420] S. A. Larin, T. van Ritbergen and J. A. M. Vermaseren, Phys. Lett. B 404 (1997) 153 hep-ph/9702435.

[421] K. Chetyrkin et al., to appear.

[422] K. Gottfried, Phys. Rev. Lett. 18 (1967) 1174.

[423] D. J. Broadhurst, A. L. Kataev and C. J. Maxwell, Phys. Lett. B 590, 76 (2004) [hep-ph/0403037). 
[424] J. Blümlein, B. Geyer and D. Robaschik, Nucl. Phys. B 560 (1999) 283 hep-ph/9903520.

[425] J. Blümlein and D. Robaschik, Phys. Rev. D 65 (2002) 096002 [hep-ph/0202077.

[426] E. V. Shuryak and A. I. Vainshtein, Nucl. Phys. B 201 (1982) 141.

[427] A. P. Bukhvostov, E. A. Kuraev and L. N. Lipatov, Sov. J. Nucl. Phys. 38 (1983) 263 [Yad. Fiz. 38 (1983) 439]; JETP Lett. 37 (1983) 482 [Pisma Zh. Eksp. Teor. Fiz. 37 (1983) 406]; [Sov. Phys. JETP 60 (1984) 22] [Zh. Eksp. Teor. Fiz. 87 (1984) 37]; Sov. J. Nucl. Phys. 39 (1984) 121 [Yad. Fiz. 39 (1984) 194];

P. G. Ratcliffe, Nucl. Phys. B 264 (1986) 493;

I. I. Balitsky and V. M. Braun, Nucl. Phys. B 311 (1989) 541;

X. D. Ji and C.-H. Chou, Phys. Rev. D 42 (1990) 3637;

J. Kodaira, Y. Yasui and T. Uematsu, Phys. Lett. B 344 (1995) 348 hep-ph/9408354];

J. Kodaira, Y. Yasui, K. Tanaka and T. Uematsu, Phys. Lett. B 387 (1996) 855 hep-ph/9603377.

[428] A. Ali, V. M. Braun and G. Hiller, Phys. Lett. B 266 (1991) 117.

[429] V. M. Braun, G. P. Korchemsky and A. N. Manashov, Phys. Lett. B 476 (2000) 455 hep-ph/0001130.

[430] B. Geyer, D. Müller and D. Robaschik, Nucl. Phys. (Proc. Suppl.) 51C (1996) 106 hep-ph/9606320.

[431] D. Müller, Phys. Lett. B 407 (1997) 314 hep-ph/9701338.

[432] J. Kodaira, S. Matsuda, T. Muta, K. Sasaki and T. Uematsu, Phys. Rev. D 20 (1979) 627. J. Kodaira, S. Matsuda, K. Sasaki and T. Uematsu, Nucl. Phys. B 159 (1979) 99;

X. -D. Ji, W. Lu, J. Osborne and X. -T. Song, Phys. Rev. D 62 (2000) 094016 hep-ph/0006121.

[433] J. Kodaira, Nucl. Phys. B 165 (1980) 129;

A. V. Belitsky, X. -D. Ji, W. Lu and J. Osborne, Phys. Rev. D 63 (2001) 094012 [hep-ph/0007305.

[434] J. Kodaira, T. Nasuno, H. Tochimura, K. Tanaka and Y. Yasui, Prog. Theor. Phys. 99 (1998) 315 hep-ph/9712395.

[435] V. M. Braun, G. P. Korchemsky and A. N. Manashov, Nucl. Phys. B 597 (2001) 370 hep-ph/0010128.

[436] V. M. Braun, G. P. Korchemsky and A. N. Manashov, Nucl. Phys. B 603 (2001) 69 hep-ph/0102313.

[437] V. M. Braun, T. Lautenschlager, A. N. Manashov and B. Pirnay, Phys. Rev. D 83 (2011) 094023 arXiv:1103.1269 [hep-ph]].

[438] M. Göckeler, R. Horsley, W. Kurzinger, H. Oelrich, D. Pleiter, P. E. L. Rakow, A. Schäfer and G. Schierholz, Phys. Rev. D 63 (2001) 074506 hep-lat/0011091.

[439] D. Dolgov et al. [LHPC and TXL Collaborations], Phys. Rev. D 66 (2002) 034506 hep-lat/0201021.

[440] A. D. Martin, R. G. Roberts, W. J. Stirling and R. S. Thorne, Phys. Lett. B 531 (2002) 216 hep-ph/0201127.

[441] S. Gottlieb, Nucl. Phys. B 139 (1978) 125; PhD thesis A New Twist in Deep Inelastic Scattering, Princeton University, 1978; Nucl. Phys. B 139 (1978) 125;

C. S. Lam and M. A. Walton, Can. J. Phys. 63 (1985) 1042.

[442] H. D. Politzer, Nucl. Phys. B 172 (1980) 349;

M. Okawa, Nucl. Phys. B 172 (1980) 481; 187 (1981) 71;

S. Wada, Nucl. Phys. B 202, 201 (1982); Phys. Lett. B 119 (1982) 427;

S. P. Luttrell, S. Wada and B. R. Webber, Nucl. Phys. B 188 (1981) 219;

S. P. Luttrell and S. Wada, Nucl. Phys. B 197 (1982) 290 [Erratum-ibid. B 206 (1982) 497];

E. V. Shuryak and A. I. Vainshtein, Phys. Lett. B 105 (1981) 65; Nucl. Phys. B 199 (1982) 451. 
[443] R. L. Jaffe and M. Soldate, Phys. Lett. B 105 (1981) 467; Phys. Rev. D 26 (1982) 49;

R. L. Jaffe, Nucl. Phys. B 229 (1983) 205.

[444] R. K. Ellis, W. Furmanski and R. Petronzio, Nucl. Phys. B 207 (1982) 1; Nucl. Phys. B 212 (1983) 29.

[445] A. P. Bukhvostov and G. V. Frolov, Yad. Fiz. 45 (1987) 1136, Sov. J. Nucl. Phys. 45 (1987) 704.

[446] J. -W. Qiu, Phys. Rev. D 42 (1990) 30.

[447] J. Bartels, C. Bontus and H. Spiesberger, hep-ph/9908411.

[448] V. Braun, R. J. Fries, N. Mahnke and E. Stein, Nucl. Phys. B 589 (2000) 381 [Erratum-ibid. B 607 (2001) 433] [hep-ph/0007279].

[449] V. M. Braun, A. N. Manashov and J. Rohrwild, Nucl. Phys. B 807 (2009) 89 [arXiv:0806.2531 [hep-ph]]; Nucl. Phys. B 826 (2010) 235 arXiv:0908.1684 [hep-ph]].

[450] M. J. Glatzmaier, S. Mantry and M. J. Ramsey-Musolf, arXiv:1208.2998 [hep-ph].

[451] V. M. Braun and A. V. Kolesnichenko, Nucl. Phys. B 283 (1987) 723;

I. I. Balitsky, V. M. Braun and A. V. Kolesnichenko, JETP Lett. 50 (1989) 61 [Pisma Zh. Eksp. Teor. Fiz. 50 (1989) 54]; Phys. Lett. B 242 (1990) 245 [Erratum-ibid. B 318 (1993) 648] hep-ph/9310316].

[452] M. Beneke, Phys. Rept. 317 (1999) 1 [hep-ph/9807443].

[453] M. Beneke and V. M. Braun, In: M. Shifman, M. (ed.): At the frontier of particle physics, 3, 1719, (World Scientific, Singapore, 2001), hep-ph/0010208.

[454] F. Eisele, M. Glück, E. Hoffmann and E. Reya, Phys. Rev. D 26 (1982) 41;

K. Varvell et al., [BEBC WA59 Collaboration], Z. Phys. C 36 (1987) 1;

M. Virchaux and A. Milsztajn, Phys. Lett. B 274 (1992) 221;

A. L. Kataev, A. V. Kotikov, G. Parente and A. V. Sidorov, Phys. Lett. B 417 (1998) 374 hep-ph/9706534;

S. I. Alekhin and A. L. Kataev, Phys. Lett. B 452 (1999) 402 [hep-ph/9812348];

M. Botje, Eur. Phys. J. C 14 (2000) 285 hep-ph/9912439;

S. I. Alekhin, Phys. Rev. D 63 (2001) 094022 hep-ph/0011002];

S. I. Alekhin, S. A. Kulagin and S. Liuti, Phys. Rev. D 69 (2004) 114009 hep-ph/0304210];

S. I. Alekhin, S. A. Kulagin and R. Petti, AIP Conf. Proc. 967 (2007) 215 [arXiv:0710.0124 [hep-ph]].

[455] J. Blümlein and H. Böttcher, Phys. Lett. B 662 (2008) 336 [arXiv:0802.0408 [hep-ph]].

[456] E. Leader, A. V. Sidorov and D. B. Stamenov, Phys. Rev. D 75 (2007) 074027 hep-ph/0612360].

[457] J. J. Aubert et al., [European Muon Collaboration], Phys. Lett. B 123 (1983) 275.

[458] A. Bodek and J. L. Ritchie, Phys. Rev. D 23 (1981) 1070; Phys. Rev. D 24 (1981) 1400.

[459] K. Rith, Ann. Phys. ( N.Y.) 176 (1987) 344; Z. Phys. C 38 (1988) 317;

D. F. Geesaman, K. Saito and A. W. Thomas, Ann. Rev. Nucl. Part. Sci. 45 (1995) 337;

P. R. Norton, Rept. Prog. Phys. 66 (2003) 1253;

N. Armesto, J. Phys. G 32 (2006) R367 hep-ph/0604108.

[460] S. Kumano, Phys. Rev. C 48 (1993) 2016 hep-ph/9303306;

M. Hirai, S. Kumano and T. -H. Nagai, Phys. Rev. C 76 (2007) 065207 [arXiv:0709.3038 [hep-ph]];

M. Hirai, S. Kumano, K. Saito and T. Watanabe, Phys. Rev. C 83 (2011) 035202 arXiv:1008.1313 [hep$\mathrm{ph}]$;

K. J. Eskola, H. Paukkunen and C. A. Salgado, JHEP 0807 (2008) 102 [arXiv:0802.0139 [hep-ph]]; Nucl. Phys. A 855 (2011) 150 arXiv:1011.6534 [hep-ph]];

I. Schienbein, et al., Phys. Rev. D 77 (2008) 054013 [arXiv:0710.4897 [hep-ph]];

Phys. Rev. D 80 (2009) 094004 [arXiv:0907.2357 [hep-ph]]. 
[461] K. J. Eskola, H. Paukkunen and C. A. Salgado, JHEP 0904 (2009) 065 arXiv:0902.4154 [hep-ph]].

[462] S. A. Kulagin and R. Petti, Nucl. Phys. A 765 (2006) 126 hep-ph/0412425.

[463] S. A. Kulagin and R. Petti, Phys. Rev. C 82 (2010) 054614 arXiv:1004.3062 [hep-ph]].

[464] D. de Florian and R. Sassot, Phys. Rev. D 69 (2004) 074028 hep-ph/0311227.

[465] D. de Florian, R. Sassot, P. Zurita and M. Stratmann, Phys. Rev. D 85 (2012) 074028 arXiv:1112.6324 [hep-ph]].

[466] J. Arrington, J. G. Rubin and W. Melnitchouk, arXiv:1110.3362 [hep-ph].

[467] R. Machleidt, K. Holinde and C. Elster, Phys. Rept. 149 (1987) 1.

[468] M. Lacombe et al., Phys. Rev. C 21 (1980) 861.

[469] S. A. Kulagin and R. Petti, Phys. Rev. D 76 (2007) 094023 hep-ph/0703033.

[470] D. M. Alde et al., Phys. Rev. Lett. 64 (1990) 2479.

[471] J. A. M. Vermaseren, Comput. Phys. Commun. 83 (1994) 45. 